\title{
D-Branes, RR-Fields and Duality on Noncommutative Manifolds
}

\author{
Jacek Brodzki ${ }^{1}$, Varghese Mathai $^{2}$, Jonathan Rosenberg ${ }^{3}$, Richard J. Szabo ${ }^{4}$ \\ ${ }^{1}$ School of Mathematics, University of Southampton, Southampton SO17 1BJ, UK. \\ E-mail: j.brodzki@ soton.ac.uk \\ 2 Department of Pure Mathematics, University of Adelaide, Adelaide 5005, Australia. \\ E-mail: mathai.varghese@ adelaide.edu.au \\ 3 Department of Mathematics, University of Maryland, College Park, MD 20742, USA. \\ E-mail: jmr@math.umd.edu \\ 4 Department of Mathematics and Maxwell Institute for Mathematical Sciences, \\ Heriot-Watt University, Riccarton, Edinburgh EH14 4AS, UK. E-mail: R.J.Szabo@ma.hw.ac.uk
}

Received: 5 July 2006 / Accepted: 19 June 2007

Published online: 5 December 2007 - (C) Springer-Verlag 2007

\begin{abstract}
We develop some of the ingredients needed for string theory on noncommutative spacetimes, proposing an axiomatic formulation of T-duality as well as establishing a very general formula for $\mathrm{D}$-brane charges. This formula is closely related to a noncommutative Grothendieck-Riemann-Roch theorem that is proved here. Our approach relies on a very general form of Poincaré duality, which is studied here in detail. Among the technical tools employed are calculations with iterated products in bivariant K-theory and cyclic theory, which are simplified using a novel diagram calculus reminiscent of Feynman diagrams.
\end{abstract}

\section{Contents}

Introduction . . . . . . . . . . . . . . . . . . . . . 644

1. D-Branes and Ramond-Ramond Charges . . . . . . . . . . . . . . . 645

1.1 Flat D-branes . . . . . . . . . . . . . . . . . . . . 6646

1.2 Ramond-Ramond fields . . . . . . . . . . . . . . . . . . . . . . . 649

1.3 Noncommutative D-branes . . . . . . . . . . . . . . . . . . . 652

1.4 Twisted D-branes . . . . . . . . . . . . . . . . . . . . . . 654

2. Poincaré Duality . . . . . . . . . . . . . . . . . . . . 656

2.1 Exterior products in K-theory . . . . . . . . . . . . . . . . 657

2.2 KK-theory . . . . . . . . . . . . . . . . 658

2.3 Strong Poincaré duality . . . . . . . . . . . . . . . . 660

2.4 Duality groups . . . . . . . . . . . . . . . . . . . . . . . . 662

2.5 Spectral triples . . . . . . . . . . . . . . . . . . . . 664

2.6 Twisted group algebra completions of surface groups . . . . . . . . 666

2.7 Other notions of Poincaré duality . . . . . . . . . . . . . . 667

3. KK-Equivalence . . . . . . . . . . . . . . . . . . . . . . . . . . . 669

3.1 Strong KK-equivalence . . . . . . . . . . . . . . . . . . . . 669

3.2 Other notions of KK-equivalence . . . . . . . . . . . . . 671 
3.3 Universal coefficient theorem . . . . . . . . . . . . . . . . . . . . . 672

3.4 Deformations . . . . . . . . . . . . . . . . . . . . 673 673

3.5 Homotopy equivalence . . . . . . . . . . . . . . . . . . 673

4. Cyclic Theory . . . . . . . . . . . . . . . . . . . . . . . . . . 673

4.1 Formal properties of cyclic homology theories . . . . . . . . . . 673

4.2 Local cyclic theory . . . . . . . . . . . . . . . . . . 675

5. Duality in Bivariant Cyclic Cohomology . . . . . . . . . . . . 678

5.1 Poincaré duality . . . . . . . . . . . . . . . . . . . 678

5.2 HL-Equivalence . . . . . . . . . . . . . . . . . . . . . . . . . . . . . 679

5.3 Spectral triples . . . . . . . . . . . . . . . . . 680

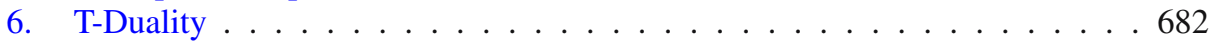

6.1 Duality for crossed products . . . . . . . . . . . . . . . . 682

6.2 T-duality and KK-equivalence . . . . . . . . . . . . . . . . . 684

$6.3 \mathrm{~T}$-duality and $\mathrm{HL}$-equivalence . . . . . . . . . . . . . . . . . . 686

7. Todd Classes and Gysin Maps . . . . . . . . . . . . . . . . . . . . . 687

7.1 The Todd class . . . . . . . . . . . . . . . . . . . . . . . . . 687

7.2 Gysin homomorphisms . . . . . . . . . . . . . . . . . . . . 690

7.3 Strongly K-oriented maps . . . . . . . . . . . . . . . . . . . . . . . 691

7.4 Weakly K-oriented maps . . . . . . . . . . . . . . . . . . . . . . 692

7.5 Grothendieck-Riemann-Roch formulas: the Strong case . . . . . . . . 693

7.6 Grothendieck-Riemann-Roch formulas: the Weak case . . . . . . . 695

8. Noncommutative D-Brane Charges . . . . . . . . . . . . . . . 695

8.1 Poincaré pairings . . . . . . . . . . . . . . . . 696

8.2 D-Brane charge formula for noncommutative spacetimes . . . . . . 697

Appendix A. The Kasparov Product . . . . . . . . . . . . . . . . . . . . 700

Appendix B. A Diagram Calculus for the Kasparov Product . . . . . . . . . . . 701

Acknowledgments . . . . . . . . . . . . . . . . . 703

References . . . . . . . . . . . . . . . . . 703

\section{Introduction}

As proposed by [63] and elaborated in [37,44,64,66,86], D-brane charges and RR-fields in string theory are classified by the K-theory of spacetime $X$, or equivalently by the K-theory of the $C^{*}$-algebra $C_{0}(X)$ of continuous functions on $X$ vanishing at infinity. Recently, in a far-sighted suggestion at KITP, I.M. Singer suggested working out string theory and duality on spacetimes that are general noncommutative $C^{*}$-algebras, with some minimal assumptions. This paper can be viewed as a preliminary step towards this goal. Some of our main results are a formula for the charges of D-branes in noncommutative spacetime and a fairly complete treatment of a general framework for T-duality. The main technical tools are a study of Poincaré duality in both KK-theory and bivariant cyclic theories, a definition of Gysin ("wrong-way") maps, and a version of the Grothendieck-Riemann-Roch theorem.

Previous work ([9,59], among many other references) already showed that a good formulation of T-duality requires the use of noncommutative algebras. We develop a formalism for dealing with T-duality in the context of general separable $C^{*}$-algebras and in Sect. 6 we give an axiomatic definition. This includes the requirement that the RR-fields and D-brane charges of $\mathcal{A}$ should be in bijective correspondence with the RRfields and D-brane charges of the T-dual $\mathrm{T}(\mathcal{A})$, and that T-duality applied twice yields a $C^{*}$-algebra which is physically equivalent to the $C^{*}$-algebra that we started out with. 
This general T-duality formalism can be viewed as a noncommutative version of the [topological aspects of the] Fourier-Mukai transform.

In the classical case, D-brane charges are expressed in terms of the Chern character in K-homology (see [75]), as formulated topologically by the Baum-Douglas construction of [6]. In formulating the notions of D-brane charges and RR-fields on arbitrary $C^{*}$ algebras, one is faced with the problem of developing Poincaré duality and constructing characteristic classes in this general setting. In [21], Connes initiated this study, pointing out that the analogue of a $\operatorname{spin}^{c}$ structure for a $C^{*}$-algebra $\mathcal{A}$ is a fundamental class $\Delta$ for its K-theory, whereas the analogue of a spin structure is a fundamental class for its KO-theory. In [65], Moscovici gives an elegant application of Poincaré duality, deriving an analogue of the Vafa-Witten inequalities for spectral triples that implement Poincaré duality, under a finite topological type hypothesis. One of the goals of this paper is to define the Todd class and Todd genus for a $\operatorname{spin}^{c} C^{*}$-algebra $\mathcal{A}$, which generalize the notion of the classical Todd class and Todd genus of a compact $\operatorname{spin}^{c}$ manifold $X$. If $\Delta$ is a fundamental class for the K-theory of the $\operatorname{spin}^{c} C^{*}$-algebra $\mathcal{A}$ and $\Xi$ is a fundamental class in bivariant cyclic homology of $\mathcal{A}$ (which is the analogue of an orientation for a smooth manifold), then we define in Sect. 7.1, the Todd class of $\mathcal{A}$ to be the invertible element

$$
\operatorname{Todd}(\mathcal{A})=\Xi^{\vee} \otimes_{\mathcal{A}^{\circ}} \operatorname{ch}(\Delta)
$$

in bivariant cyclic homology of the algebra $\mathcal{A}$. (The notations are explained below; $\Xi^{\vee}$ is the dual fundamental class to $\Xi$ and $\mathcal{A}^{\circ}$ is the opposite algebra to $\mathcal{A}$.) In the special case when $\mathcal{A}$ is a spin $C^{*}$-algebra and the K-theory fundamental class $\Delta$ comes from a fundamental class in KO-theory, the characteristic class as defined above is called the Atiyah-Hirzebruch class $\widehat{A}(\mathcal{A})$. One of our main results, Theorem 7.10 , shows that the Todd class as defined above is exactly the correction factor needed in the noncommutative Grothendieck-Riemann-Roch formula.

Our final main result, the D-brane charge formula of Sect. 8.2, is a noncommutative analogue of the well-known formula (1.1) in [63] (cf. [44,64,66,86]). It takes the familiar form,

$$
\mathrm{Q}_{\xi}=\operatorname{ch}\left(f_{!}(\xi)\right) \otimes_{\mathcal{A}} \sqrt{\operatorname{Todd}(\mathcal{A})},
$$

for a $\mathrm{D}$-brane $\mathcal{B}$ in a noncommutative spacetime $\mathcal{A}$ with given weakly K-oriented morphism $f: \mathcal{A} \rightarrow \mathcal{B}$ and Chan-Paton bundle $\xi \in \mathrm{K}_{\bullet}(\mathcal{B})$, where $f_{\text {! }}$ denotes the Gysin map associated to $f$. With this modification of the Chern character, one obtains an isometry between the natural intersection pairings in K-theory and cyclic theory of $\mathcal{A}$. There is also a similar dual formula for the charge of a D-brane given by a Fredholm module, representing the Chern-Simons coupling of D-branes with RR-fields.

The central mathematical technique of the paper is the development of a novel diagram calculus for KK theory and the analogous diagram calculus for bivariant cyclic theory, in Appendix B. The rules of this diagram calculus are reminiscent of those for the calculus of Feynman diagrams, and are likely to become an important tool for simplifying iterated sequences of intersection products in KK-theory and in cyclic theory, and for establishing identities in these theories.

\section{D-Branes and Ramond-Ramond Charges}

In this section we give a detailed mathematical description of brane charges in the language of topological K-homology and singular cohomology. Our aim later on is then 
to generalize these constructions to analytic K-homology and cyclic cohomology suitable to generic noncommutative settings, some examples of which we describe below. For a description of D-branes in terms of K-theory see $[63,64,66,86]$, and in terms of K-homology see $[1,75,84]$.

1.1. Flat $D$-branes. Let $X$ be a spin manifold of dimension $d=10$ with metric. In Type II superstring theory, $X$ is called the spacetime. If $X$ is non-compact, appropriate compact support conditions are always implicitly understood throughout. In our later applications we can typically relax some of these requirements and only assume that $X$ is a finite-dimensional Hausdorff space which has the homotopy type of a finite CW-complex.

Definition 1.1. A flat D-brane in $X$ is a triple $(W, E, \phi)$, where $\phi: W \hookrightarrow X$ is a closed, embedded $\operatorname{spin}^{c}$ submanifold and $E \in \mathrm{K}^{0}(W)$. The submanifold $W$ is called the worldvolume and the class E the Chan-Paton bundle of the D-brane.

When $E$ is the stable isomorphism class of a complex vector bundle over $W$, we assume that it is equipped with a connection and refer to the triple as a brane system. When $E$ is only a virtual bundle, say $E=E^{+}-E^{-}$, we can loosely regard it as the class of a complex $\mathbb{Z}_{2}$-graded bundle $E^{+} \oplus E^{-}$equipped with a superconnection and the triple is called a brane-antibrane system. The requirement that a D-brane $(W, E, \phi)$ be invariant under processes involving brane-antibrane creation and annihilation is the statement of stable isomorphism of Chan-Paton bundles. Physical quantities which are invariant under deformations of $E$ thereby depend only on its K-theory class in $\mathrm{K}^{0}(W)$ [86]. Deformation invariance, gauge symmetry enhancement and the possibility of branes within branes then imply that any D-brane $(W, E, \phi)$ should be subjected to the usual equivalence relations of topological K-homology [6], i.e., bordism, direct sum and vector bundle modification, respectively [75]. We will not distinguish between a D-brane and its $\mathrm{K}$-homology class in $\mathrm{K}_{\bullet}(X)$, nor between the Chan-Paton bundle and its isomorphism class in $\mathrm{K}^{0}(W)$.

To define the charge of a flat D-brane in the spacetime manifold $X$, we begin by introducing a natural bilinear pairing on the K-theory of $X$,

$$
\langle-,-\rangle: \mathrm{K}^{0}(X) \times \mathrm{K}^{0}(X) \stackrel{\otimes}{\longrightarrow} \mathrm{K}^{0}(X) \stackrel{\operatorname{index}\left(D_{(-)}\right)}{\longrightarrow} \mathbb{Z},
$$

where $D_{N}: C^{\infty}\left(X, \mathrm{~S}_{X}^{+} \otimes N\right) \rightarrow C^{\infty}\left(X, \mathrm{~S}_{X}^{-} \otimes N\right)$ is the twisted Dirac operator on $X$, with respect to a chosen connection on the complex vector bundle $N \rightarrow X$, and $\mathrm{S}_{X}^{ \pm} \rightarrow X$ are the two half-spinor bundles over $X$. When tensored over $\mathbb{Q}$ the pairing (1.1) is nondegenerate, which is equivalent to Poincaré duality in rational K-theory. In the topological setting, Poincaré duality is generically determined by the bilinear cap product

$$
\mathrm{K}^{0}(X) \otimes \mathrm{K}_{\bullet}(X) \stackrel{\cap}{\longrightarrow} \mathrm{K}_{\bullet}(X)
$$

defined for any complex vector bundle $F \rightarrow X$ and any D-brane $(W, E, \phi)$ in $X$ by

$$
F \cap(W, E, \phi)=\left(W, E \otimes \phi^{*} F, \phi\right) .
$$

The index pairing $\mathrm{K}^{0}(X) \otimes \mathrm{K}_{\bullet}(X) \rightarrow \mathbb{Z}$ is then provided by the Dirac operator on $W$ as

$$
F \otimes(W, E, \phi) \longmapsto \operatorname{index}\left(D_{E \otimes \phi^{*} F}\right) .
$$


On the other hand, in cohomology the natural bilinear pairing is given by the intersection form

$$
(-,-): \mathrm{H}^{p}(X, \mathbb{Z}) \times \mathrm{H}^{d-p}(X, \mathbb{Z}) \stackrel{\cup}{\longrightarrow} \mathrm{H}^{d}(X, \mathbb{Z}) \stackrel{(-)[X]}{\longrightarrow} \mathbb{Z} .
$$

Again nondegeneracy of this pairing over $\mathbb{Q}$ is equivalent to the Poincaré duality theorem of classical rational cohomology theory [7, p. 44]. For a compact oriented manifold $X$, the pairing between cohomology groups of complementary degrees leads to the duality

$$
\mathrm{H}^{p}(X, \mathbb{Q}) \cong\left(\mathrm{H}^{d-p}(X, \mathbb{Q})\right)^{\vee} \cong \mathrm{H}_{d-p}(X, \mathbb{Q}) .
$$

It is important to realize that this pairing is determined purely in terms of the topology of $X$, while the index pairing between K-theory and K-homology uses both the knowledge of the topology of $X$ and the analysis of the Dirac operator $\not D$. This difference will become important when we compare the two pairings using the Chern character below. The statement of cohomological Poincaré duality in the non-oriented case requires the use of twisted coefficients, while in K-theory the Poincaré pairing involves twisting whenever $X$ is not $\operatorname{spin}^{c}$. This links very importantly with twisted K-theory [3].

Recall that the Atiyah-Hirzebruch class $\widehat{A}(X)$ of the manifold $X$ is invertible with respect to the cup product on cohomology [55, p. 257]. An application of the AtiyahSinger index theorem (recalling that $X$ is spin) then immediately gives the following fundamental result.

Proposition 1.2. The modified Chern isomorphism

$$
\mathrm{Ch}: \mathrm{K}^{0}(X) \otimes \mathbb{Q} \longrightarrow \mathrm{H}^{\text {even }}(X, \mathbb{Q})=\bigoplus_{n \geq 0} \mathrm{H}^{2 n}(X, \mathbb{Q})
$$

defined by

$$
\operatorname{Ch}(N)=\operatorname{ch}(N) \cup \widehat{A}(X)^{1 / 2}
$$

is an isometry with respect to the natural inner products (1.1) and (1.5),

$$
\left\langle N, N^{\prime}\right\rangle=\left(\mathrm{Ch}(N), \operatorname{Ch}\left(N^{\prime}\right)\right) .
$$

Note that the ordinary Chern character ch preserves the addition and multiplication on K-theory and cohomology, but not the bilinear forms. The modified Chern character $\mathrm{Ch}$ preserves addition but not the cup products. A similar statement is also true for the Chern character on $\mathrm{K}^{-1}(X) \otimes \mathbb{Q} \rightarrow \mathrm{H}^{\text {odd }}(X, \mathbb{Q})=\bigoplus_{n \geq 0} \mathrm{H}^{2 n+1}(X, \mathbb{Q})$. However, because of the suspension isomorphism $\mathrm{K}^{-1}(X) \cong \mathrm{K}^{0}(X \times \mathbb{R})$ it will suffice to work explicitly with $\mathrm{K}^{0}$ groups alone in the following. In string theory terms this means that we work only with Type IIB D-branes, the analogous results for Type IIA branes being obtainable by T-duality (see Sect. 6).

There is an elementary but useful alternative interpretation of Proposition 1.2. Since the $\widehat{A}$-class is an even degree (inhomogeneous) class in the cohomology ring $\mathrm{H}^{\text {even }}(X$, $\mathbb{Q})$, with non-zero constant term, its square root $\widehat{A}(X)^{1 / 2}$ is also invertible. It follows that taking products with this class produces an isomorphism $\mathfrak{h}: \mathrm{H}^{\text {even }}(X, \mathbb{Q}) \rightarrow$ $\mathrm{H}^{\text {even }}(X, \mathbb{Q})$ which is given explicitly by

$$
\omega \longmapsto \omega \cup \widehat{A}(X)^{1 / 2} .
$$


When we combine this isomorphism with the pairing given by Poincaré duality, we obtain a new nondegenerate pairing $(-,-)_{\mathfrak{h}}: \mathrm{H}^{\text {even }}(X, \mathbb{Q}) \times \mathrm{H}^{\text {even }}(X, \mathbb{Q}) \rightarrow \mathbb{R}$ defined by

$$
\begin{aligned}
\left(\alpha, \alpha^{\prime}\right)_{\mathfrak{h}} & :=\left(\alpha \cup \widehat{A}(X)^{1 / 2}, \alpha^{\prime} \cup \widehat{A}(X)^{1 / 2}\right) \\
& =\left(\alpha \cup \widehat{A}(X)^{1 / 2} \cup \alpha^{\prime} \cup \widehat{A}(X)^{1 / 2}\right)[X] \\
& =\left(\alpha \cup \alpha^{\prime} \cup \widehat{A}(X)\right)[X],
\end{aligned}
$$

where we have used commutativity of the cup product. It is now easy to see that the classical Chern character is an isometry with respect to the two pairings (1.1) and (1.11),

$$
\left\langle N, N^{\prime}\right\rangle=\left(\operatorname{ch}(N), \operatorname{ch}\left(N^{\prime}\right)\right)_{\mathfrak{h}} .
$$

From this point of view the isomorphism $\mathfrak{h}$ transforms the purely topological pairing $(-,-)$ to the "index" pairing $(-,-)_{\mathfrak{h}}$, where the latter contains the information about the extra piece of index machinery given by the Atiyah-Hirzebruch class.

For any closed oriented embedding $\phi: W \hookrightarrow X$ of dimension $p$, we denote by $[W]$ its orientation cycle in $\mathrm{H}_{p}(X, \mathbb{Z})$, by $\operatorname{Pd}_{X}(W)=\operatorname{Pd}_{W \hookrightarrow X}=\left([X] \cap \_\right)^{-1}[W]$ its Poincaré dual in $\mathrm{H}^{d-p}(X, \mathbb{Z})$, and by $\phi_{!}: \mathrm{K}^{\bullet}(W) \rightarrow \mathrm{K}^{\bullet+d-p}(X)$ the corresponding K-theoretic Gysin homomorphism. Recall that on cohomology, the Gysin map is given explicitly by $\phi_{!}=\mathrm{Pd}_{X} \circ \phi_{*} \circ \mathrm{Pd}_{W}^{-1}: \mathrm{H}^{\bullet}(W, \mathbb{Z}) \rightarrow \mathrm{H}^{\bullet+d-p}(X, \mathbb{Z})$.

Definition 1.3. The Ramond-Ramond charge (RR-charge for short) of a D-brane ( $W, E$, $\phi)$ in $X$ is the modified Chern characteristic class $\mathrm{Ch}\left(\phi_{!} E\right) \in \mathrm{H}^{\bullet}(X, \mathbb{Q})$. If $\left(W^{\prime}, E^{\prime}, \phi^{\prime}\right)$ is any other D-brane in $X$, then the $\left(W^{\prime}, E^{\prime}, \phi^{\prime}\right)$-charge of $(W, E, \phi)$ is the integer

$$
Q_{W^{\prime}, E^{\prime}, \phi^{\prime}}(W, E, \phi)=\left(\operatorname{Pd}_{X}\left(W^{\prime}\right), \operatorname{Ch}\left(\phi_{!} E\right)\right)=\phi^{\prime *} \operatorname{Ch}\left(\phi_{!} E\right)\left[W^{\prime}\right] .
$$

When $\left(W^{\prime}, E^{\prime}, \phi^{\prime}\right)=(W, E, \phi)$, we write simply $Q_{W, E, \phi}=Q_{W, E, \phi}(W, E, \phi)$ for the charge of the D-brane $(W, E, \phi)$ itself. Note that this charge formula for a D-brane is written entirely in terms of spacetime quantities.

Let us momentarily assume, for simplicity, that the spacetime manifold $X$ is compact. Let $C(X)$ denote the $C^{*}$-algebra of continuous complex-valued functions on $X$. A standard construction in K-homology then provides the following result.

Proposition 1.4. There is a one-to-one correspondence between flat D-branes in X, modulo Baum-Douglas equivalence, and stable homotopy classes of Fredholm modules over the algebra $C(X)$.

Proof. Consider a D-brane $(W, E, \phi)$ such that $\operatorname{dim}(W)$ is odd. The worldvolume $W$ inherits a metric from $X$ and its Chan-Paton bundle $E$ is equipped with a (super)connection $\nabla$. Let $\mathrm{S}_{W} \rightarrow W$ be the spinor bundle over $W$, and consider the usual twisted Dirac operator $D_{E}: C^{\infty}\left(W, \mathrm{~S}_{W} \otimes E\right) \rightarrow C^{\infty}\left(W, \mathrm{~S}_{W} \otimes E\right)$ with respect to the chosen connection $\nabla$. Using the metric we can complete the vector space of smooth sections $C^{\infty}\left(W, \mathrm{~S}_{W} \otimes E\right)$ of the twisted spinor bundle and view $D_{E}: \mathcal{H} \rightarrow \mathcal{H}$ as an unbounded self-adjoint Fredholm operator on the separable Hilbert space

$$
\mathcal{H}=L^{2}\left(W, \mathrm{~S}_{W} \otimes E\right)
$$


Let us now define a unital algebra $*$-homomorphism $\rho: C(X) \rightarrow \mathcal{B}(\mathcal{H})$ as pointwise multiplication on $\mathcal{H}$ via

$$
\rho(f)=m_{f \circ \phi} \otimes \mathbb{1}_{\mathrm{S}_{W} \otimes E}, \quad \forall f \in C(X),
$$

where $m_{g}: C(W) \rightarrow C(W)$ is the pointwise multiplication operator $h \mapsto g h$. Since the Dirac operator is closable, we can thereby form an odd Fredholm module $(\mathcal{H}, \rho, F)$ over the algebra $C(X)$, where $F=D_{E} /\left|D_{E}\right|$ is the partial isometry in the polar decomposition of $D_{E}$. Similarly, when $W$ is even-dimensional, we can form an even Fredholm module $(\mathcal{H}, \rho, F)$, where the $\mathbb{Z}_{2}$-grading $\mathcal{H}=\mathcal{H}^{+} \oplus \mathcal{H}^{-}$on the Hilbert space (1.14) is given by

$$
\mathcal{H}^{ \pm}=L^{2}\left(W, \mathrm{~S}_{W}^{ \pm} \otimes E\right)
$$

with $\mathrm{S}_{W}^{ \pm} \rightarrow W$ the two half-spinor bundles over $W$, the odd bounded Fredholm operator $F$ constructed as above from the corresponding twisted Dirac operator $\not_{E}$ : $C^{\infty}\left(W, \mathrm{~S}_{W}^{+} \otimes E\right) \rightarrow C^{\infty}\left(W, \mathrm{~S}_{W}^{-} \otimes E\right)$, and the even $*$-homomorphism $\rho: C(X) \rightarrow$ $\mathcal{B}\left(\mathcal{H}^{ \pm}\right)$defined as in (1.15). The clases of the Fredholm modules built in this way are independent of the choice of metric on $X$ and connection $\nabla$ on $E$.

Conversely, allow arbitrary coefficient classes in K-theory (this requires certain care with the defining equivalence relations $[48,75])$. Then the K-homology class of the cycle $\left(X, E, \mathrm{id}_{X}\right)$ is the Poincare dual of $E$, which can be any class in $\mathrm{K}_{\bullet}(X)$. We conclude that all classes in the $\mathrm{K}_{\text {-homology }} \mathrm{K}^{\bullet}(\mathcal{A})=\mathrm{KK}_{\bullet}(\mathcal{A}, \mathbb{C})$ of the algebra $\mathcal{A}=C(X)$ can be obtained by using an appropriate $\mathrm{D}$-brane.

Proposition 1.4 of course simply establishes the equivalence between the analytic and topological descriptions of K-homology. Any Fredholm module over the $C^{*}$-algebra $C(X)$ is therefore a flat D-brane in the spacetime $X$. The usefulness of this point of view is that it can be extended to more general brane configurations (that we describe in the following) which are represented by noncommutative algebras. Namely, a D-brane may be generically regarded as the homotopy class of a suitable Fredholm module over an algebra $\mathcal{A}$. In what follows we will reformulate the description of D-brane charge in the language of cyclic cocycles. This will require, in particular, an analytic reformulation of the natural pairings introduced above. More precisely, one of our main goals in this paper is to provide a generic, noncommutative version of the result (1.12).

1.2. Ramond-Ramond fields. Closely related to the definition of D-brane charge given above is the notion of a Ramond-Ramond field. In what follows we use the cup product $\cup$ when multiplying together cohomology classes, and exterior products $\wedge$ when multiplying arbitrary differential forms. In a similar vein to what we have done before, we will not distinguish between (co)homology classes and their explicit representatives.

Let Fred $=$ Fred $(\mathcal{H})$ be the space of Fredholm operators on a separable Hilbert space $\mathcal{H}$. Then Fred is a classifying space for K-theory of $X$ and any vector bundle $E \rightarrow X$ can be obtained as the index bundle of a map into Fred. Let $\mathrm{c}$ be a choice of cocycle representative for the universal Chern character. If $f_{E}: X \rightarrow$ Fred is the classifying map of a bundle $E \rightarrow X$, then $\operatorname{ch}(E)=f_{E}^{*} \mathrm{c} \in \mathrm{H}^{\text {even }}(X, \mathbb{R})$.

Consider triples $(f, C, \omega)$, where $f \in[X$, Fred], $\omega$ is an inhomogeneous form of even degree, and $C$ is an inhomogeneous cochain of odd degree satisfying

$$
\mathrm{d} C=\omega-f^{*} \mathrm{c} .
$$


The collection of all such triples is denoted $\mathfrak{K}^{0}(X)$. Two elements $\left(f_{0}, C_{0}, \omega_{0}\right)$ and $\left(f_{1}, C_{1}, \omega_{1}\right)$ of $\mathfrak{K}^{0}(X)$ are called equivalent if there is a triple $(f, C, \omega)$ on $X \times[0,1]$, with $\omega$ constant on $\{x\} \times[0,1]$ for each $x \in X$, such that $\left.(f, C, \omega)\right|_{X \times\{0\}}=\left(f_{0}, C_{0}, \omega_{0}\right)$ and $\left.(f, C, \omega)\right|_{X \times\{1\}}=\left(f_{1}, C_{1}, \omega_{1}\right)$. The set of equivalence classes forms an abelian group under addition of triples called the differential $\mathrm{K}$-theory group $\breve{\mathrm{K}}^{0}(X)$, cf. $\S 4$ in [43]. It fits into the short exact sequence

$$
0 \longrightarrow \mathrm{K}^{-1}(X) \otimes \mathbb{R} / \mathbb{Z} \longrightarrow \breve{\mathrm{K}}^{0}(X) \longrightarrow \mathrm{A}^{0}(X) \longrightarrow 0,
$$

where $A^{0}(X)$ is defined by the pullback square

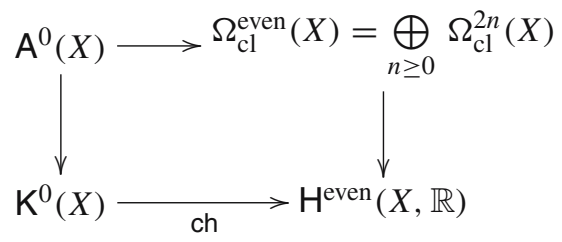

with $\Omega_{\mathrm{cl}}^{2 n}(X)$ the space of closed $2 n$-forms on $X$.

The RR-fields (of Type IIA superstring theory) are closed even degree forms associated to elements of $\mathfrak{K}^{0}(X)[36,64]$.

Definition 1.5. The Ramond-Ramond field (RR-field for short) $G$ associated to an element of $\mathfrak{K}^{0}(X)$ which maps to $(E, \omega) \in \mathrm{A}^{0}(X)$ under (1.18) is the closed differential form

$$
G(E, \omega)=\omega \wedge \widehat{A}(X)^{1 / 2}
$$

The topological equivalence class of the RR-field is the D-brane charge regarded as an element of the appropriate K-theory group. The D-branes "couple" to RR-fields, and another way to define D-brane charge is through the pairings of their characteristic classes with these differential forms.

Definition 1.6. The Chern-Simons coupling of a D-brane $(W, E, \phi)$ to an RR-field corresponding to the element $(f, C, \omega) \in \mathfrak{K}^{0}(X)$ is the spacetime integral

$$
S_{\mathrm{CS}}(W, E, \phi \mid C)=\int_{X} C \wedge \mathrm{Ch}\left(\phi_{!} E\right) .
$$

Given this notion, we can now formulate an alternative homological definition of D-brane charge.

Definition 1.7. The dual Ramond-Ramond charge (dual RR-charge for short) of a D-brane $(W, E, \phi)$ in $X$ is the rational homology class $\mathrm{Ch}(W, E, \phi) \in \mathrm{H}_{\bullet}(X, \mathbb{Q})$ such that

$$
S_{\mathrm{CS}}(W, E, \phi \mid C)=\int_{\mathrm{Ch}(W, E, \phi)} C
$$

for all RR-fields on $X$. 
Evidently, the natural framework for the Chern-Simons couplings of D-branes is K-homology. The Chern character in topological K-homology is the isomorphism

$$
\text { ch }: \mathrm{K}_{\bullet}(X) \otimes \mathbb{Q} \longrightarrow \mathrm{H}_{\bullet}(X, \mathbb{Q})
$$

defined by

$$
\operatorname{ch}(W, E, \phi)=\phi_{*} \circ \operatorname{Pd}_{W}^{-1}(\operatorname{ch}(E) \cup \operatorname{Todd}(W))
$$

for any D-brane $(W, E, \phi)$ in $X$. The Todd class is related to the Atiyah-Hirzebruch class by

$$
\operatorname{Todd}(W)=\mathrm{e}^{-\mathrm{d}(W)} \cup \widehat{A}(W)
$$

where $\mathrm{d}(W) \in \mathrm{H}^{2}(W, \mathbb{Z})$ is a characteristic class whose reduction modulo 2 is the second Stiefel-Whitney class $\mathbf{w}_{2}(W) \in \mathrm{H}^{2}\left(W, \mathbb{Z}_{2}\right)$. This specifies the $\operatorname{spin}^{c}$ structure on the brane worldvolume $W$ as follows. The $\operatorname{spin}^{c}$ groups $\operatorname{Spin}^{c}(n)=\operatorname{Spin}(n) \times_{\mathbb{Z}_{2}} U(1)$ fit into a commutative diagram

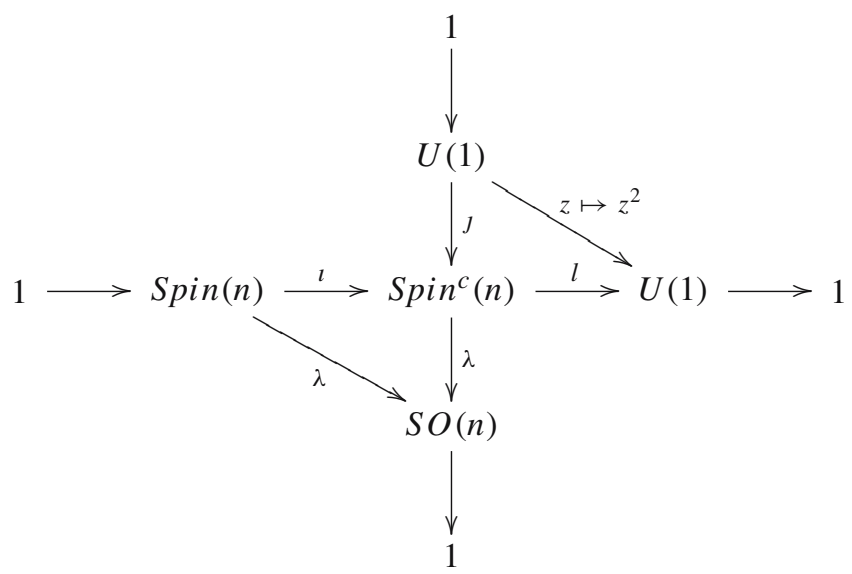

whose row and column are exact sequences. The map $\lambda: \operatorname{Spin}(n) \rightarrow S O(n)$ is the universal cover of the group $S O(n)$, while $j: U(1) \hookrightarrow \operatorname{Spin}^{c}(n)$ and $l: \operatorname{Spin}(n) \hookrightarrow$ $\operatorname{Spin}^{c}(n)$ are natural inclusions. The homomorphism $l: \operatorname{Spin}^{c}(n) \rightarrow U(1)$ is defined by $(g, z) \mapsto z^{2}$. It induces a map $\mathrm{H}^{1}\left(W, \operatorname{Spin}^{c}(n)\right) \rightarrow \mathrm{H}^{1}(W, U(1))$ and thus we may associate a complex line bundle $L \rightarrow W$ with the worldvolume $W$. The corresponding Chern class is the characteristic class $\mathrm{d}(W):=\mathrm{C}_{1}(L)$.

The homological Chern character preserves sums, as well as the cap product in the sense that

$$
\operatorname{ch}(F \cap(W, E, \phi))=\operatorname{ch}(F) \cap \operatorname{ch}(W, E, \phi)
$$

for any complex vector bundle $F \rightarrow X$. This follows from its definition (1.24), the multiplicativity of the cohomological Chern character, the index theorem, and the Atiyah-Hirzebruch version of the Riemann-Roch theorem,

$$
\phi_{!}(\operatorname{ch}(E) \cup \operatorname{Todd}(W))=\operatorname{ch}\left(\phi_{!} E\right) \cup \widehat{A}(X),
$$


which together give

$$
\begin{aligned}
\operatorname{ch}(F) \diamond \operatorname{ch}(W, E, \phi) & =\operatorname{ch}(F) \cup \phi_{!}(\operatorname{ch}(E) \cup \operatorname{Todd}(W))[X] \\
& =\operatorname{ch}\left(F \otimes \phi_{!} E\right) \cup \widehat{A}(X)[X] \\
& =\operatorname{index}\left(\not D_{F \otimes \phi_{!} E}\right)
\end{aligned}
$$

with $\diamond: \mathrm{H}^{\bullet}(X, \mathbb{Z}) \times \mathrm{H}_{\bullet}(X, \mathbb{Z})$ the pairing between cohomology and homology. This is just the index pairing (1.4).

As the notation suggests, the dual charge of a D-brane is a modification of the homological Chern character analogous to the modification in the case of cohomology.

Proposition 1.8. The dual RR-charge $\mathrm{Ch}(W, E, \phi) \in \mathrm{H}_{\bullet}(X, \mathbb{Q})$ of a D-brane $(W, E, \phi)$ in $X$ can be represented by

$$
\operatorname{Ch}(W, E, \phi)=\operatorname{Pd}_{X}^{-1}\left(\operatorname{Pd}_{X} \circ \operatorname{ch}(W, E, \phi) \cup \widehat{A}(X)^{-1 / 2}\right)
$$

Proof. We use (1.28) along with (1.8) to rewrite the D-brane charge as

$$
\operatorname{Ch}\left(\phi_{!} E\right)=\phi_{!}(\operatorname{ch}(E) \cup \operatorname{Todd}(W)) \cup \widehat{A}(X)^{-1 / 2}
$$

Along with the definition (1.24), we can use (1.31) to rewrite the Chern-Simons coupling (1.21) in the form

$$
S_{\mathrm{CS}}(W, E, \phi \mid C)=\int_{X} C \wedge\left(\mathrm{Pd}_{X} \circ \operatorname{ch}(W, E, \phi) \cup \widehat{A}(X)^{-1 / 2}\right) .
$$

By comparing this with the definition (1.22) of the dual charge, (1.30) follows.

1.3. Noncommutative D-branes. There are many sorts of noncommutative D-branes, i.e., D-branes modeled as Fredholm modules over a noncommutative algebra, and here we will discuss only a few special instances. To motivate the first generalization of our definition of a D-brane given above, we look at an alternative way of regarding the embedding $\phi: W \hookrightarrow X$ of a flat D-brane into spacetime. Consider a tubular neighbourhood $W^{\prime}$ of $W$ in $X$. For any point $u \in W$, there is an isomorphism $T_{u} X \cong T_{u} W \oplus N_{u}(X / W)$, where $N(X / W) \rightarrow W$ is the normal bundle, which can be identified with $\phi^{*}(T X) / T W$, in terms of the proper differentiable map $\phi: W \hookrightarrow X$. Let $\Psi: W^{\prime} \rightarrow N(X / W)$ be the diffeomorphism which identifies the normal bundle $N(X / W)$ with the tubular neighbourhood $W^{\prime}$. Then $\widehat{\phi}:=\Psi \circ \phi$ is the zero section of $N(X / W) \rightarrow W$, and in this way we may identify the embedding of the worldvolume into spacetime as a smooth section of the corresponding normal bundle, $\widehat{\phi} \in C^{\infty}(W, N(X / W))$.

Definition 1.9. A flat nonabelian D-brane in $X$ is a quadruple $(W, E, \phi, \widehat{\phi})$, where $\phi$ : $W \hookrightarrow X$ is a closed, embedded $\operatorname{spin}^{c}$ submanifold, $E \in \mathrm{K}^{0}(W)$, and $\widehat{\phi} \in C^{\infty}(W, N(X / W) \otimes \operatorname{End}(E))$. 
When $E$ is a complex line bundle, we identify $\widehat{\phi}=\phi$ and in this case the nonabelian D-brane is the same object that we defined above. Nonabelian D-branes are classified by the same K-theory as abelian ones. In general, the algebra $\mathcal{A}=C(X) \otimes C^{\infty}(W, \operatorname{End}(E))$ acts on the Hilbert spaces (1.14) or (1.16), and so one can formulate this definition in the language of Fredholm modules over an algebra which is Morita equivalent to $C(X)$.

A related notion arises within the framework of Fredholm modules when one replaces the algebra of functions on spacetime with an appropriate noncommutative algebra.

Definition 1.10. A flat noncommutative D-brane in $X$ is a Fredholm module over a deformation $\mathcal{A}_{\theta}$ of the algebra $\mathcal{A}=C(X)$.

For the most part, noncommutative D-branes are classified by the same K-theory as commutative ones. However, this assumes that $\mathrm{K}$-theory is preserved under deformation [79], which is not always the case. See [56] for an interesting counterexample.

Example 1.11. Consider $X=\mathbb{R}^{2 n}$ (with compactly-supported cohomology groups), and let $\mathcal{S}\left(\mathbb{R}^{2 n}\right)$ be the space of complex Schwartz functions on $\mathbb{R}^{2 n}$. Let $\theta=\left(\theta^{i j}\right)$ be a real, invertible skew-symmetric $2 n \times 2 n$ matrix. For $f, g \in \mathcal{S}\left(\mathbb{R}^{2 n}\right)$, we define the corresponding twisted product

$$
f \star_{\theta} g(x):=(2 \pi)^{-2 n} \iint f\left(x-\frac{1}{2} \theta u\right) g(x+v) \mathrm{e}^{-\mathrm{i} u \cdot v} \mathrm{~d}^{2 n} u \mathrm{~d}^{2 n} v,
$$

where $\mathrm{d}^{2 n} u$ is the Lebesgue measure on $\mathbb{R}^{2 n}$. The deformed algebra $\mathcal{A}_{\theta}$ is then defined as

$$
\mathcal{A}_{\theta}=\left(\mathcal{S}\left(\mathbb{R}^{2 n}\right), \star_{\theta}\right) .
$$

This is an associative Fréchet algebra which defines a noncommutative space that is often called the Moyal n-plane or noncommutative Euclidean space. D-branes may be constructed analogously to the commutative case. For instance, for $f \in \mathcal{A}_{\theta}$ let $m_{f}^{\theta}: \mathcal{A}_{\theta} \rightarrow$ $\mathcal{A}_{\theta}$ denote the left multiplication operator $g \mapsto f \star_{\theta} g$, and let $\mathcal{H}=L^{2}\left(\mathbb{R}^{2 n}\right) \otimes \mathbb{C}^{2^{n}}$ be the Hilbert space of ordinary square-integrable spinors on $\mathbb{R}^{2 n}$. Let $\not D$ be the ordinary Euclidean Dirac operator, and define a $*$-representation $\rho^{\theta}: \mathcal{A}_{\theta} \rightarrow \mathcal{B}(\mathcal{H})$ by $\rho^{\theta}(f)=$ $m_{f}^{\theta} \otimes \mathbb{1}_{2^{n}}$. Then $\left(\mathcal{H}, \rho^{\theta}, F\right)$, with $F=\not D /|\not D|$, is a Fredholm module over the algebra (1.34).

Example 1.12. Let $X$ be a closed Riemannian spin manifold equipped with a smooth isometric action of a $2 n$-torus $\mathbb{T}^{2 n}$. The periodic action of $\mathbb{T}^{2 n}$ on $X$ induces by pullback an action of $\mathbb{T}^{2 n}$ by automorphisms $\tau$ on the algebra $\mathcal{A}=C^{\infty}(X)$ of smooth functions on $X$. The orbits on which $\mathbb{T}^{2 n}$ acts freely determine maps $\sigma_{s}: C^{\infty}(X) \rightarrow C^{\infty}\left(\mathbb{T}^{2 n}\right)$. Let $\mathcal{T}_{\theta}^{2 n}:=\left(C^{\infty}\left(\mathbb{T}^{2 n}\right), \star_{\theta}\right)$ be the noncommutative torus defined as the algebra of smooth functions on the ordinary torus endowed with the periodised version of the twisted product (1.33). Pulling back this deformation by the maps $\sigma_{s}$ gives rise to an algebra $\mathcal{A}_{\theta}:=\left(C^{\infty}(X), \times_{\theta}\right)$. This defines a broad class of noncommutative spaces known as toric noncommutative manifolds. The product $f \times_{\theta} g$ is given by a periodic twisted product just like (1.33), with the non-periodic translations replaced by the periodic $\mathbb{T}^{2 n}$ action. Alternatively, $\mathcal{A}_{\theta}$ may be defined as the fixed point subalgebra of $C^{\infty}(X) \hat{\otimes} \mathcal{T}_{\theta}^{2 n}$ under the action of the automorphism $\tau \otimes \tau^{-1}$ (with $\hat{\otimes}$ the projective tensor product of Fréchet algebras). The construction of D-branes in these cases again parallels that of the commutative case and Example 1.11 above. 
The special classes of noncommutative branes given by Examples 1.11 and 1.12 above will be referred to as isospectral deformations of flat D-branes. Other interesting examples may be found in [23] and [22].

1.4. Twisted D-branes. A very important instance in which noncommutative D-branes arise is through the formulation of the notion of a curved D-brane. These arise when the spacetime manifold $X$ carries certain topologically non-trivial characteristics in the following sense. Recall that a gerbe over $X$ is an infinite rank principal bundle over $X$ with projective unitary structure group and characteristic class $H$. A gerbe connection is a Deligne cohomology class on $X$ with top form $H$.

Definition 1.13. A B-field $(X, H)$ is a gerbe with one-connection over $X$ and characteristic class $H \in \mathrm{H}^{3}(X, \mathbb{Z})$ called an NS-NS H-flux.

For any oriented submanifold $W \subset X$, we denote by $\mathrm{W}_{3}(W) \in \mathrm{H}^{3}(W, \mathbb{Z})$ the third integer Stiefel-Whitney class of its normal bundle $N(X / W)$. It is the obstruction to a $\operatorname{spin}^{c}$ structure on $W$.

Definition 1.14. A curved or twisted D-brane in a $B$-field $(X, H)$ is a triple $(W, E, \phi)$, where $\phi: W \hookrightarrow X$ is a closed, embedded oriented submanifold with $\phi^{*} H=W_{3}(W)$, and $E \in \mathrm{K}^{0}(W)$.

The condition on the brane embedding is required to cancel the global Freed-Witten anomalies [37] arising in the worldsheet functional integral. Suitable equivalence classes of curved D-branes take values in the twisted topological $\mathrm{K}$-homology $\mathrm{K}_{\bullet}(X, H)$ [84]. For $H=0$, the worldvolume $W$ is $\operatorname{spin}^{c}$ and the definition reduces to that of the flat case. One should also require that the brane worldvolume $W$ carry a certain projective structure that reduces for $H=0$ to the usual characteristic class $\mathrm{d}(W) \in \mathrm{H}^{2}(W, \mathbb{Z})$ specifying a $\operatorname{spin}^{c}$ structure on $W$.

A $B$-field can be realized by a bundle of algebras over $X$ whose sections define a noncommutative $C^{*}$-algebra. When $H \in \operatorname{Tor}\left(\mathrm{H}^{3}(X, \mathbb{Z})\right)$ is a torsion class, this is known as an Azumaya algebra bundle [13]. Via the Sen-Witten construction, D-branes in $(X, H)$ may then be realized in terms of $n \mathrm{D} 9$ brane-antibrane pairs carrying a principal $S U(n) / \mathbb{Z}_{n}=U(n) / U(1)$ Chan-Paton bundle. Cancellation of anomalies then requires $n H=0$. To accommodate non-torsion characteristic classes, one must consider a certain $n \rightarrow \infty$ limit which can be realized as follows.

Let us fix a separable Hilbert space $\mathcal{H}$, and denote by $P U(\mathcal{H})=U(\mathcal{H}) / U(1)$ the group of projective unitary automorphisms of $\mathcal{H}$. Let $\mathcal{K}(\mathcal{H})$ be the $C^{*}$-algebra of compact operators on $\mathcal{H}$. For any $g \in U(\mathcal{H})$, the map $\operatorname{Ad}_{g}: \mathcal{K}(\mathcal{H}) \rightarrow \mathcal{K}(\mathcal{H})$ defined by $\operatorname{Ad}_{g}(T)=g T g^{-1}$ is an automorphism. The assignment $g \mapsto \operatorname{Ad}_{g}$ defines a continuous epimorphism Ad $: U(\mathcal{H}) \rightarrow \operatorname{Aut}(\mathcal{K}(\mathcal{H}))$ with respect to the strong operator topology on $U(\mathcal{H})$ and the point-norm topology on $\operatorname{Aut}(\mathcal{K}(\mathcal{H}))$ with $\operatorname{ker}(\operatorname{Ad})=U(1)$. It follows that one can identify the group $P U(\mathcal{H})$ with $\operatorname{Aut}(\mathcal{K}(\mathcal{H}))$ under this homomorphism.

The exact sequence of sheaves of germs of continuous functions on $X$ given by

$$
1 \longrightarrow \underline{U(1)}_{X} \longrightarrow \underline{U(\mathcal{H})}_{X} \longrightarrow \underline{P U(\mathcal{H})}_{X} \longrightarrow 1
$$

induces a long exact sequence of sheaf cohomology groups as

$$
\longrightarrow \mathrm{H}^{1}\left(X, \underline{U(\mathcal{H})}_{X}\right) \longrightarrow \mathrm{H}^{1}\left(X, \underline{P U(\mathcal{H})}_{X}\right) \stackrel{\delta_{1}}{\longrightarrow} \mathrm{H}^{2}(X, \underline{U(1)} X) \longrightarrow
$$


Since the unitary group $U(\mathcal{H})$ is contractible with respect to the strong operator topology, the sheaf $\frac{U(\mathcal{H})}{X}$ is soft and so $\mathrm{H}^{j}\left(X, \underline{U(\mathcal{H})}_{X}\right)=0$ for all $j \geq 1$. It follows that the map $\delta_{1}$ is an isomorphism. From the exact sequence of groups

$$
1 \longrightarrow \mathbb{Z} \longrightarrow \mathbb{R} \longrightarrow U(1) \longrightarrow 1
$$

we obtain the long exact cohomology sequence

$$
\longrightarrow \mathrm{H}^{2}\left(X, \underline{\mathbb{R}}_{X}\right) \longrightarrow \mathrm{H}^{2}(X, \underline{U(1)} X) \stackrel{\delta_{2}}{\longrightarrow} \mathrm{H}^{3}(X, \mathbb{Z}) \longrightarrow \mathrm{H}^{3}\left(X, \underline{\mathbb{R}}_{X}\right) \longrightarrow .
$$

Again, since $\underline{\mathbb{R}}_{X}$ is a fine sheaf, one has $\mathrm{H}^{j}\left(X, \underline{\mathbb{R}}_{X}\right)=0$ for all $j \geq 1$ and so the map $\delta_{2}$ is an isomorphism.

The map

$$
\delta_{X}=\delta_{2} \circ \delta_{1}: \mathrm{H}^{1}(X, \underline{P U(\mathcal{H})} X) \longrightarrow \mathrm{H}^{3}(X, \mathbb{Z})
$$

is thus an isomorphism on stable equivalence classes of principal $P U(\mathcal{H})$-bundles over the spacetime $X$. If $P \rightarrow X$ is a $P U(\mathcal{H})$-bundle and $[P] \in \mathrm{H}^{1}\left(X, P U(\mathcal{H})_{X}\right)$ is its isomorphism class, then $\delta_{X}(P):=\delta_{X}([P]) \in \mathrm{H}^{3}(X, \mathbb{Z})$ is called the DixmierDouady invariant of $P[13,15,61]$. The set of isomorphism classes of locally trivial bundles over $X$ with structure group $\operatorname{Aut}(\mathcal{K}(\mathcal{H}))$ and fibre $\mathcal{K}(\mathcal{H})$ form a group $\mathrm{Br}^{\infty}(X)$ under tensor product called the infinite Brauer group of $X$. Using the identification $P U(\mathcal{H}) \cong \operatorname{Aut}(\mathcal{K}(\mathcal{H}))$, it follows that such algebra bundles are also classified by $\mathrm{H}^{3}(X, \mathbb{Z})$. If $\mathcal{E}$ is a bundle of this kind, then the corresponding element of $\mathrm{H}^{3}(X, \mathbb{Z})$ is also called the Dixmier-Douady invariant of $\mathcal{E}[61]$ and denoted $\delta_{X}(\mathcal{E})$.

Given a $B$-field $(X, H)$, there corresponds a unique, locally trivial $C^{*}$-algebra bundle $\mathcal{E}_{H} \rightarrow X$ with fibre $\mathcal{K}(\mathcal{H})$ and structure group $P U(\mathcal{H})$ whose Dixmier-Douady invariant is

$$
\delta_{X}\left(\mathcal{E}_{H}\right)=H .
$$

Let $C_{0}\left(X, \varepsilon_{H}\right)$ be the $C^{*}$-algebra of continuous sections, vanishing at infinity, of this algebra bundle. The twisted $\mathrm{K}$-theory $\mathrm{K}^{\bullet}(X, H)=\mathrm{K}_{\bullet}\left(C_{0}\left(X, \mathcal{E}_{H}\right)\right)[3,78]$ may then be computed as the set of stable homotopy classes of sections of an associated algebra bundle $P_{H} \times{ }_{P U(\mathcal{H})} \operatorname{Fred}(\mathcal{H})$, where $P_{H}$ is a principal $P U(\mathcal{H})$-bundle over $X$ and $\operatorname{Fred}(\mathcal{H})$ is the algebra of (self-adjoint) Fredholm operators on $\mathcal{H}$ with $P U(\mathcal{H})$ acting by conjugation. On the other hand, one can define Dixmier-Douady classes over any D-brane worldvolume $W$ in complete analogy with (1.38) and show that [69]

$$
\mathrm{W}_{3}(W)=\delta_{W}(\operatorname{Cliff}(N(X / W))),
$$

where $\operatorname{Cliff}(N(X / W)) \rightarrow W$ is the Clifford algebra bundle of the normal bundle $N(X / W)$. The Dixmier-Douady class $\delta_{W}(\operatorname{Cliff}(N(X / W)))$ is the global obstruction to existence of a spinor bundle $\mathrm{S}_{W}$ with

$$
\operatorname{Cliff}_{p}(N(X / W)) \cong \operatorname{End}_{p}\left(\mathrm{~S}_{W}\right)
$$

for $p \in W$. This observation leads to the following result.

Proposition 1.15. There is a one-to-one correspondence between twisted D-branes in $(X, H)$ and stable homotopy classes of Fredholm modules over the algebra $C_{0}\left(X, \mathcal{E}_{H}\right)$. 
The proof of Proposition 1.15 fixes the appropriate equivalence relations required for twisted topological K-homology. One of these equivalence relations (in addition to the appropriate twisted analogs of bordism, direct sum and vector bundle modification) is based on the observation [57] that while a triple $\left(W^{\prime}, E^{\prime}, \phi^{\prime}\right)$ may violate the embedding condition of a curved D-brane, one can still cancel the Freed-Witten anomalies on the submanifold $W^{\prime}$ by adding a "source" in $W^{\prime}$ corresponding to a twisted D-brane. This D-brane can be unstable and decay due to the configuration $\left(W^{\prime}, E^{\prime}, \phi^{\prime}\right)$. This physical process can be stated more precisely as follows.

Lemma 1.16 (Stabilization). Let $(W, E, \phi)$ be a twisted D-brane in $(X, H)$ whose orientation cycle $[W]$ is non-trivial in $\mathrm{H}_{\bullet}(X, \mathbb{Z})$. Suppose that there exists a closed, embedded oriented submanifold $\phi^{\prime}: W^{\prime} \hookrightarrow X$ such that $W$ is a codimension 3 submanifold of $W^{\prime}$ and its Poincaré dual $\mathrm{Pd}_{W \hookrightarrow W^{\prime}}$ satisfies the equation

$$
\phi^{\prime *} H=\mathrm{W}_{3}\left(W^{\prime}\right)+\mathrm{Pd}_{W \hookrightarrow W^{\prime}}
$$

in $\mathrm{H}^{3}\left(W^{\prime}, \mathbb{Z}\right)$. Then $(W, E, \phi)$ is trivial in $\mathrm{K}_{\bullet}(X, H)$ (up to twisted vector bundle modification).

The structure of D-branes in torsion $B$-fields simplifies drastically. When $H \in$ $\operatorname{Tor}\left(\mathrm{H}^{3}(X, \mathbb{Z})\right)$ the algebra $C_{0}\left(X, \varepsilon_{H}\right)$ is Morita equivalent to an Azumaya algebra bundle over $X$, i.e., a bundle whose fibres are Azumaya algebras with local trivializations reducing them to $n \times n$ matrix algebras $\mathbb{M}_{n}(\mathbb{C})$. Two Azumaya bundles $\mathcal{E}, \mathcal{F}$ over $X$ are called equivalent if there are vector bundles $E, F$ over $X$ such that $\mathcal{E} \otimes \operatorname{End}(E)$ is isomorphic to $\mathcal{F} \otimes \operatorname{End}(F)$. The set of equivalence classes is a group $\operatorname{Br}(X)$ under tensor product called the Brauer group of $X$. There is also a notion of Dixmier-Douady invariant $\delta_{X}^{\prime}$ for Azumaya bundles over $X$, which is constructed using the same local description as above but now with $\mathcal{H}$ a finite-dimensional complex vector space. By Serre's theorem one has $\operatorname{Br}(X) \cong \operatorname{Tor}\left(\mathrm{H}^{3}(X, \mathbb{Z})\right)$. This gives two descriptions of $\operatorname{Tor}\left(\mathrm{H}^{3}(X, \mathbb{Z})\right)$, one in terms of locally trivial bundles over $X$ with fibre $\mathcal{K}(\mathcal{H})$ and structure group $\operatorname{Aut}(\mathcal{K}(\mathcal{H}))$, and the other in terms of Azumaya bundles. They are related by the following result from [61].

Proposition 1.17. If $X$ is a compact manifold and $\mathcal{E}$ is a locally trivial bundle over $X$ with fibre $\mathcal{K}(\mathcal{H})$ and structure group $\operatorname{Aut}(\mathcal{K}(\mathcal{H}))$, then the algebra $C(X, \mathcal{E})$ is stably unital if and only if its Dixmier-Douady invariant is a torsion element in $\mathrm{H}^{3}(X, \mathbb{Z})$.

These constructions allow us to describe the K-theory of the noncommutative $C^{*}$-algebra $C\left(X, \mathcal{E}_{H}\right)$ [61]. Morita equivalence induces an isomorphism between the K-theories of $C_{0}\left(X, \mathcal{E}_{H}\right)$ and $C_{0}\left(X, \mathcal{A}_{H}\right)$, where $\mathcal{A}_{H}$ is an Azumaya bundle associated to $\varepsilon_{H}$ via the Dixmier-Douady invariant [13]. A geometric description of this K-theory is provided by the notion of projective vector bundle [61], while in the infinite-dimensional setting of a non-torsion $B$-field one needs to introduce the notions of bundle gerbes and bundle gerbe modules [8].

\section{Poincaré Duality}

A crucial point of our construction of flat D-brane charges in Sect. 1.1 was the role played by Poincaré duality. With an eye to generalizing the construction to the more general settings described above, in this section we will explore how and to what extent this classical notion of topology can be generalized to generic $C^{*}$-algebras in the context 
of KK-theory. We will describe various criteria which guarantee the duality. There are several natural inequivalent versions of Poincaré duality, which we define and study, giving many purely noncommutative examples. Our examples range from those of classical spaces to noncommutative deformations of $\operatorname{spin}^{c}$ manifolds, and also the more general examples of Poincaré duality spaces such as those arising in the case of the free group acting on its boundary or, more generally, for hyperbolic groups acting on their Gromov boundaries.

2.1. Exterior products in $\mathrm{K}$-theory. To describe Poincaré duality generically in K-theory, we first need to make some important remarks concerning the product structure. Let $\mathcal{A}_{1}$ and $\mathcal{A}_{2}$ be unital $C^{*}$-algebras. If $p_{1} \in \mathbb{M}_{k}\left(\mathcal{A}_{1}\right)$ is a projection representing a Murrayvon Neumann equivalence class in $\mathrm{K}_{0}\left(\mathcal{A}_{1}\right)$ and a projection $p_{2} \in \mathbb{M}_{l}\left(\mathcal{A}_{2}\right)$ represents a class in $\mathrm{K}_{0}\left(\mathcal{A}_{2}\right)$, then the tensor product $p_{1} \otimes p_{2}$ is a projection in $\mathbb{M}_{k}\left(\mathcal{A}_{1}\right) \otimes \mathbb{M}_{l}\left(\mathcal{A}_{2}\right) \cong$ $\mathbb{M}_{k l}\left(\mathcal{A}_{1} \otimes \mathcal{A}_{2}\right)$ for any $C^{*}$-tensor product and so it represents a class in $\mathrm{K}_{0}\left(\mathcal{A}_{1} \otimes \mathcal{A}_{2}\right)$ (in this section we will work mostly with the maximal tensor product). In this way we obtain a map

$$
\mathrm{K}_{0}\left(\mathcal{A}_{1}\right) \times \mathrm{K}_{0}\left(\mathcal{A}_{2}\right) \longrightarrow \mathrm{K}_{0}\left(\mathcal{A}_{1} \otimes \mathcal{A}_{2}\right)
$$

This definition extends to non-unital algebras in a standard way [41, p. 104]. In the special case $\mathcal{A}_{1}=\mathcal{A}_{2}=\mathcal{A}$ we obtain a map

$$
\mathrm{K}_{0}(\mathcal{A}) \times \mathrm{K}_{0}(\mathcal{A}) \longrightarrow \mathrm{K}_{0}(\mathcal{A} \otimes \mathcal{A})
$$

It is important to note that, in contrast to the topological case, it is not possible in general to make $\mathrm{K}_{0}(\mathcal{A})$ into a ring. We recall that for a compact topological space $X$ there is an exterior product map

$$
\mathrm{K}^{0}(X) \times \mathrm{K}^{0}(X) \longrightarrow \mathrm{K}^{0}(X \times X)
$$

which is defined using the exterior tensor product of vector bundles. The diagonal map $X \rightarrow X \times X$ induces a natural transformation $\mathrm{K}^{0}(X \times X) \rightarrow \mathrm{K}^{0}(X)$. The composition of the two maps thereby leads to the product

$$
\mathrm{K}^{0}(X) \times \mathrm{K}^{0}(X) \longrightarrow \mathrm{K}^{0}(X) .
$$

If $\mathcal{A}=C(X)$ is the algebra of continuous functions on $X$, then the diagonal map translates into the product map

$$
m: \mathcal{A} \otimes \mathcal{A} \longrightarrow \mathcal{A}
$$

on the algebra $\mathcal{A}$ given by $m(a \otimes b)=a b$ for all $a, b \in \mathcal{A}$. Since $\mathcal{A}$ is commutative, the multiplication (2.5) is an algebra homomorphism and there is an induced map

$$
m_{*}: \mathrm{K}_{0}(\mathcal{A} \otimes \mathcal{A}) \longrightarrow \mathrm{K}_{0}(\mathcal{A}) \text {. }
$$

For a noncommutative $C^{*}$-algebra the multiplication map is not an algebra homomorphism and so we cannot expect that in general the map (2.6) will be defined.

Recall that the suspension of a generic $C^{*}$-algebra $\mathcal{A}$ is the $C^{*}$-algebra $\Sigma(\mathcal{A}):=$ $C_{0}(\mathbb{R}) \otimes \mathcal{A}$. By definition one has $\mathrm{K}_{p}(\mathcal{A})=\mathrm{K}_{0}\left(\Sigma^{p}(\mathcal{A})\right)=\mathrm{K}_{0}\left(C_{0}\left(\mathbb{R}^{p}\right) \otimes \mathcal{A}\right)$. Bott 
periodicity ensures that up to isomorphism there are only two distinct K-theory groups $\mathrm{K}_{0}(\mathcal{A})$ and $\mathrm{K}_{1}(\mathcal{A})$. One has

$$
\begin{aligned}
\Sigma^{k}\left(\mathcal{A}_{1}\right) \otimes \Sigma^{l}\left(\mathcal{A}_{2}\right) & =\left(C_{0}\left(\mathbb{R}^{k}\right) \otimes \mathcal{A}_{1}\right) \otimes\left(C_{0}\left(\mathbb{R}^{l}\right) \otimes \mathcal{A}_{2}\right) \\
& \cong C_{0}\left(\mathbb{R}^{k+l}\right) \otimes\left(\mathcal{A}_{1} \otimes \mathcal{A}_{2}\right) \\
& =\Sigma^{k+l}\left(\mathcal{A}_{1} \otimes \mathcal{A}_{2}\right) .
\end{aligned}
$$

If we combine this formula with the exterior product (2.1) defined for $\mathrm{K}_{0}$-groups then we obtain the general exterior product

$$
\mathrm{K}_{k}\left(\mathcal{A}_{1}\right) \times \mathrm{K}_{l}\left(\mathcal{A}_{2}\right) \longrightarrow \mathrm{K}_{k+l}\left(\mathcal{A}_{1} \otimes \mathcal{A}_{2}\right)
$$

See [41, §4.7] for more details and examples.

Many important statements in K-theory admit a concise formulation in terms of the product structure. For example, there exists a canonical class $\beta \in \mathrm{K}_{2}(\mathbb{C})=\mathrm{K}_{0}\left(C_{0}\left(\mathbb{R}^{2}\right)\right)$, called the Bott generator, such that the exterior product with $\beta$ defines a map

$$
\mathrm{K}_{0}(\mathcal{A}) \stackrel{\otimes \beta}{\longrightarrow} \mathrm{K}_{2}(\mathcal{A} \otimes \mathbb{C})=\mathrm{K}_{2}(\mathcal{A})
$$

This provides the isomorphism required by the Bott periodicity theorem [41, §4.9]. These observations all find their most natural generalisation in Kasparov's KK-theory [51], which we now proceed to describe. (See [5, Ch. VIII] for a more detailed exposition.)

2.2. KK-theory. Let $\mathcal{B}$ be a $C^{*}$-algebra. A Hilbert $\mathcal{B}$-module $\mathcal{H}$ is a module over $\mathcal{B}$ equipped with a $\mathcal{B}$-valued inner product

$$
\mathcal{H} \times \mathcal{H} \longrightarrow \mathcal{B}, \quad\left(\zeta, \zeta^{\prime}\right) \longmapsto\left\langle\zeta \mid \zeta^{\prime}\right\rangle \in \mathcal{B}
$$

which satisfies similar properties to those of an inner product with values in $\mathbb{C}$ [54]. We denote by $\mathcal{L}(\mathcal{H})$ the algebra of linear operators on $\mathcal{H}$ which admit an adjoint with respect to this inner product. The closed subalgebra generated by all rank 1 operators of the form $\theta_{\zeta, \zeta^{\prime}}: \xi \mapsto \zeta\left\langle\zeta^{\prime} \mid \xi\right\rangle$ is denoted $\mathcal{K}(\mathcal{H})$ and called the algebra of compact operators on $\mathcal{H}$. The algebra $\mathcal{K}(\mathcal{H})$ is a closed ideal in $\mathcal{L}(\mathcal{H})$.

Definition 2.1. Let $\mathcal{A}$ and $\mathcal{B}$ be $C^{*}$-algebras. An odd $\mathcal{A}-\mathcal{B}$ Kasparov bimodule is a triple $(\mathcal{H}, \rho, F)$, where $\mathcal{H}$ is a countably generated Hilbert $\mathcal{B}$-module, the map $\rho: \mathcal{A} \rightarrow \mathcal{L}(\mathcal{H})$ is $a *$-homomorphism, and $F \in \mathcal{L}(\mathcal{H})$ is a self-adjoint operator such that for each $a \in \mathcal{A}$ one has

$$
\rho(a)\left(\operatorname{id}_{\mathcal{H}}-F^{2}\right) \in \mathcal{K}(\mathcal{H}) \quad \text { and } \quad F \rho(a)-\rho(a) F \in \mathcal{K}(\mathcal{H}) .
$$

An even $\mathcal{A}-\mathcal{B}$ Kasparov bimodule is a triple $(\mathcal{H}, \rho, F)$ where $\mathcal{H}=\mathcal{H}^{+} \oplus \mathcal{H}^{-}$is $\mathbb{Z}_{2}$-graded, $\phi$ is an even degree map, $F$ is an odd map, and the compactness conditions (2.11) are satisfied. In both cases a triple is called degenerate if the operators $\rho(a)\left(\operatorname{id}_{\mathcal{H}}-F^{2}\right)$ and $F \rho(a)-\rho(a) F$ are zero for all $a \in \mathcal{A}$. 
We denote by $\mathcal{E}_{0}(\mathcal{A}, \mathcal{B})$ and $\mathcal{E}_{1}(\mathcal{A}, \mathcal{B})$ the sets of isomorphism classes of even and odd Kasparov bimodules, respectively. These two sets are made into semi-groups using the direct sum of Kasparov bimodules. Two triples $(\mathcal{H}, \rho, F)$ and $\left(\mathcal{H}, \rho^{\prime}, F^{\prime}\right)$ are regarded as equivalent if (by adding a degenerate triple to both if necessary) $F^{\prime}$ can be obtained from $F$ via operator homotopy. Imposing these equivalence relations on $\mathcal{E}_{0}(\mathcal{A}, \mathcal{B})$ and $\mathcal{E}_{1}(\mathcal{A}, \mathcal{B})$ yields two abelian groups $\mathrm{KK}_{0}(\mathcal{A}, \mathcal{B})$ and $\mathrm{KK}_{1}(\mathcal{A}, \mathcal{B})$. The functor $\mathrm{KK}_{\bullet}(\mathcal{A}, \mathcal{B})$ is homotopy invariant and satisfies excision in both variables with respect to $\mathbb{C}$-split exact sequences of $C^{*}$-algebras.

The special case where $\mathcal{B}=\mathbb{C}$ is important. A Hilbert $\mathbb{C}$-module $\mathcal{H}$ is just a Hilbert space, and the algebra $\mathcal{L}(\mathcal{H})$ is in this case the $C^{*}$-algebra of bounded linear operators on $\mathcal{H}$. The compactness conditions (2.11) provide an abstraction of the essential properties of elliptic operators [2], and a Kasparov bimodule in this case is just a Fredholm module. Thus we can define the K-homology of the $C^{*}$-algebra $\mathcal{A}$ as

$$
\mathrm{K}^{\bullet}(\mathcal{A})=\mathrm{KK}_{\bullet}(\mathcal{A}, \mathbb{C}) .
$$

One can also show that $\mathrm{KK}_{\bullet}(\mathbb{C}, \mathcal{A})$ is isomorphic to the $\mathrm{K}_{\text {-theory }} \mathrm{K}_{\bullet}(\mathcal{A})$ of the algebra $\mathcal{A}$.

The key property of the bivariant functor $\mathrm{KK}_{\bullet}(\mathcal{A}, \mathcal{B})$ is the existence of an associative product

$$
\otimes_{\mathcal{B}}: \mathrm{KK}_{i}(\mathcal{A}, \mathcal{B}) \times \mathrm{KK}_{j}(\mathcal{B}, \mathcal{C}) \longrightarrow \mathrm{KK}_{i+j}(\mathcal{A}, \mathcal{C})
$$

induced by the composition of bimodules, which is additive in both variables. This product is called the composition or intersection product and it is compatible with algebra homomorphisms in the following sense. There is a functor from the category of separable $C^{*}$-algebras to an additive category $\mathrm{KK}$ whose objects are separable $C^{*}$-algebras and whose morphisms $\mathcal{A} \rightarrow \mathcal{B}$ are precisely the elements of $\mathrm{KK}_{\bullet}(\mathcal{A}, \mathcal{B})$. An algebra homomorphism $\phi: \mathcal{A} \rightarrow \mathcal{B}$ thus defines an element $\mathrm{KK}(\phi) \in \mathrm{KK}_{0}(\mathcal{A}, \mathcal{B})$, and if $\psi: \mathcal{B} \rightarrow \mathcal{C}$ is another homomorphism then

$$
\mathrm{KK}(\psi \circ \phi)=\mathrm{KK}(\phi) \otimes_{\mathcal{B}} \mathrm{KK}(\psi) \in \mathrm{KK}_{0}(\mathcal{A}, \mathcal{C}) .
$$

The intersection product makes $\mathrm{KK}_{\bullet}(\mathcal{A}, \mathcal{A})$ into a $\mathbb{Z}_{2}$-graded ring whose unit element is $1_{\mathcal{A}}:=\mathrm{KK}\left(\mathrm{id}_{\mathcal{A}}\right)$, the element of $\mathrm{KK}_{0}(\mathcal{A}, \mathcal{A})$ determined by the identity map $\operatorname{id}_{\mathcal{A}}: \mathcal{A} \rightarrow \mathcal{A}$.

The operation of taking the composition product by a fixed element $\alpha \in \mathrm{KK}_{0}(\mathcal{A}, \mathcal{B})$ gives a map

$$
\mathrm{KK}_{i}(\mathbb{C}, \mathcal{A}) \longrightarrow \mathrm{KK}_{i}(\mathbb{C}, \mathcal{B}),
$$

i.e., a homomorphism $\alpha_{*}: \mathrm{K}_{i}(\mathcal{A}) \rightarrow \mathrm{K}_{i}(\mathcal{B})$ in $\mathrm{K}$-theory, and also a map

$$
\mathrm{KK}_{i}(\mathcal{B}, \mathbb{C}) \longrightarrow \mathrm{KK}_{i}(\mathcal{A}, \mathbb{C}),
$$

i.e., a homomorphism of $\mathrm{K}$-homology groups $\alpha^{*}: \mathrm{K}^{i}(\mathcal{B}) \rightarrow \mathrm{K}^{i}(\mathcal{A})$. If $\alpha$ is the class of a bimodule $(\mathcal{H}, \rho, F)$, then $(2.15)$ is the index map index $\operatorname{mox}_{i}: \mathrm{K}_{i}(\mathcal{A}) \rightarrow \mathrm{K}_{i}(\mathcal{B})$. In general, we will say that the element $\alpha$ is invertible if there exists $\beta \in \mathrm{KK}_{0}(\mathcal{B}, \mathcal{A})$ such that $\alpha \otimes_{\mathcal{B}} \beta=1_{\mathcal{A}} \in \operatorname{KK}_{0}(\mathcal{A}, \mathcal{A})$ and $\beta \otimes_{\mathcal{A}} \alpha=1_{\mathcal{B}} \in \mathrm{KK}_{0}(\mathcal{B}, \mathcal{B})$. We call $\beta$ the inverse of $\alpha$ and write $\beta=\alpha^{-1}$. An invertible element of $\operatorname{KK}_{0}(\mathcal{A}, \mathcal{B})$ gives an isomorphism $\mathrm{K}_{i}(\mathcal{A}) \cong \mathrm{K}_{i}(\mathcal{B})$ of $\mathrm{K}$-theory groups and of $\mathrm{K}$-homology groups $\mathrm{K}^{i}(\mathcal{A}) \cong \mathrm{K}^{i}(\mathcal{B})$. This construction will be generalized in Sect. 3.1.

The composition product (2.13), along with the natural map $\mathrm{KK}_{i}(\mathcal{A}, \mathcal{B}) \rightarrow \mathrm{KK}_{i}(\mathcal{A} \otimes$ $\mathcal{C}, \mathcal{B} \otimes \mathcal{C}$ ) given by $\alpha \mapsto \alpha \otimes 1_{\mathcal{C}}$, imply the existence of a more general associative product 
in KK-theory called the Kasparov product. For any collection of separable $C^{*}$-algebras $\mathcal{A}_{1}, \mathcal{B}_{1}, \mathcal{A}_{2}, \mathcal{B}_{2}$ and $\mathcal{D}$ there is a bilinear map

$$
\mathrm{KK}_{i}\left(\mathcal{A}_{1}, \mathcal{B}_{1} \otimes \mathcal{D}\right) \otimes_{\mathcal{D}} \mathrm{KK}_{j}\left(\mathcal{D} \otimes \mathcal{A}_{2}, \mathcal{B}_{2}\right) \longrightarrow \mathrm{KK}_{i+j}\left(\mathcal{A}_{1} \otimes \mathcal{A}_{2}, \mathcal{B}_{1} \otimes \mathcal{B}_{2}\right)
$$

This product can be thought of as a mixture between the usual cup and cap products. For $\mathcal{D}=\mathbb{C}$ it specializes to the exterior product (also written $\times$ ):

$$
\mathrm{KK}_{i}\left(\mathcal{A}_{1}, \mathcal{B}_{1}\right) \otimes \mathrm{KK}_{j}\left(\mathcal{A}_{2}, \mathcal{B}_{2}\right) \longrightarrow \mathrm{KK}_{i+j}\left(\mathcal{A}_{1} \otimes \mathcal{A}_{2}, \mathcal{B}_{1} \otimes \mathcal{B}_{2}\right)
$$

When $\mathcal{A}_{1}=\mathcal{A}_{2}=\mathbb{C},(2.18)$ is just the exterior product on $\mathrm{K}$-theory that we discussed in Sect. 2.1 above. On the other hand, when we put $\mathcal{B}_{1}=\mathbb{C}$ and $\mathcal{A}_{1}=\mathbb{C}$ in (2.17) we recover the original composition product (2.13) in the form

$$
\mathrm{KK}_{i}\left(\mathcal{A}_{1}, \mathcal{D}\right) \otimes_{\mathcal{D}} \mathrm{KK}_{j}\left(\mathcal{D}, \mathcal{B}_{2}\right) \longrightarrow \mathrm{KK}_{i+j}\left(\mathcal{A}_{1}, \mathcal{B}_{2}\right)
$$

Various technical details of the Kasparov product, useful for explicit computations, are collected in Appendix A, and a pictorial method for keeping track of these is given in Appendix B.

2.3. Strong Poincaré duality. Poincaré duality for $C^{*}$-algebras was defined by Connes $[20,21]$ in the context of real KK-theory as a means of defining noncommutative $\operatorname{spin}^{c}$ manifolds. It was subsequently extended to more general situations by Kaminker and Putnam [50], Emerson [32,33], amongst others. These latter works motivate our first definition of the duality in the context of complex KK-theory.

Definition 2.2 (Strong Poincaré Duality). A pair of separable $C^{*}$-algebras $(\mathcal{A}, \mathcal{B})$ is said to be a strong Poincaré dual pair (strong PD pair for short) if there exists a class $\Delta \in \mathrm{KK}_{d}(\mathcal{A} \otimes \mathcal{B}, \mathbb{C})=\mathrm{K}^{d}(\mathcal{A} \otimes \mathcal{B})$ in the $\mathrm{K}$-homology of $\mathcal{A} \otimes \mathcal{B}$ and a class

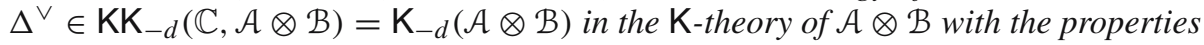

$$
\Delta^{\vee} \otimes_{\mathcal{B}} \Delta=1_{\mathcal{A}} \in \mathrm{KK}_{0}(\mathcal{A}, \mathcal{A}) \text { and } \Delta^{\vee} \otimes_{\mathcal{A}} \Delta=(-1)^{d} 1_{\mathcal{B}} \in \mathrm{KK}_{0}(\mathcal{B}, \mathcal{B}) \text {. }
$$

The element $\Delta$ is called a fundamental K-homology class for the pair $(\mathcal{A}, \mathcal{B})$ and $\Delta^{\vee}$ is called its inverse. A separable $C^{*}$-algebra $\mathcal{A}$ is said to be a strong Poincaré duality algebra (strong PD algebra for short) if $\left(\mathcal{A}, \mathcal{A}^{\mathrm{o}}\right)$ is a strong PD pair, where $\mathcal{A}^{\mathrm{o}}$ denotes the opposite algebra of $\mathcal{A}$, i.e., the algebra with the same underlying vector space as $\mathcal{A}$ but with the product reversed.

Remark 2.3. The use of the opposite algebra in this definition is to describe $\mathcal{A}$-bimodules as $\left(\mathcal{A} \otimes \mathcal{A}^{\mathrm{o}}\right)$-modules. We will see this explicitly in Sect. 2.5 below.

Let us indicate how Definition 2.2 is used to implement Poincaré duality. First of all, we note that the tensor product algebra $\mathcal{A} \otimes \mathcal{B}$ is canonically isomorphic to the algebra $\mathcal{B} \otimes \mathcal{A}$ through the "flip" map $\mathcal{A} \otimes \mathcal{B} \rightarrow \mathcal{B} \otimes \mathcal{A}$ which interchanges the two factors. Thus $\mathrm{K}^{d}(\mathcal{A} \otimes \mathcal{B}) \cong \mathrm{K}^{d}(\mathcal{B} \otimes \mathcal{A})$ and $\mathrm{K}_{-d}(\mathcal{A} \otimes \mathcal{B}) \cong \mathrm{K}_{-d}(\mathcal{B} \otimes \mathcal{A})$. With this observation, we can use the Kasparov product (2.17) to induce a map

$$
\begin{aligned}
\otimes_{\mathcal{A}}: \mathrm{KK}_{d}(\mathcal{A} \otimes \mathcal{B}, \mathbb{C}) \otimes \mathrm{K}_{i}(\mathcal{A}) \cong & \mathrm{KK}_{d}(\mathcal{B} \otimes \mathcal{A}, \mathbb{C}) \otimes \mathrm{KK}_{i}(\mathbb{C}, \mathcal{A}) \\
& \longrightarrow \mathrm{KK}_{d+i}(\mathcal{B}, \mathbb{C})=\mathrm{K}^{d+i}(\mathcal{B}) .
\end{aligned}
$$


Thus taking the product on the right with the element $\Delta \in \mathrm{KK}_{d}(\mathcal{A} \otimes \mathcal{B}, \mathbb{C})$ produces a map

$$
\mathrm{K}_{i}(\mathcal{A}) \stackrel{\otimes_{\mathcal{A}} \Delta}{\longrightarrow} \mathrm{K}^{i+d}(\mathcal{B})
$$

from the $\mathrm{K}$-theory $\mathrm{K}_{i}(\mathcal{A})$ of the algebra $\mathcal{A}$ to the $\mathrm{K}$-homology $\mathrm{K}^{i+d}(\mathcal{B})$ of the algebra $\mathcal{B}$. Since the element $\Delta$ has an inverse $\Delta^{\vee} \in \mathrm{KK}_{-d}(\mathbb{C}, \mathcal{A} \otimes \mathcal{B})$, using the exterior product again we can define a map

$$
\otimes_{\mathcal{B}}: \mathrm{KK}_{-d}(\mathbb{C}, \mathcal{A} \otimes \mathcal{B}) \otimes \mathrm{KK}_{i}(\mathcal{B}, \mathbb{C}) \longrightarrow \mathrm{KK}_{-d+i}(\mathbb{C}, \mathcal{A})=\mathrm{K}_{-d+i}(\mathcal{A}) .
$$

Thus multiplying on the left by the element $\Delta^{\vee}$ establishes a map

$$
\mathrm{K}^{i}(\mathcal{B}) \stackrel{\Delta^{\vee} \otimes_{\mathcal{B}}}{\longrightarrow} \mathrm{K}_{i-d}(\mathcal{A})
$$

from the K-homology of $\mathcal{B}$ to the K-theory of $\mathcal{A}$.

Since $\Delta$ and $\Delta^{\vee}$ are inverses to each other, for any $x \in \mathrm{K}_{i}(\mathcal{A})$ one has

$$
\begin{aligned}
\Delta^{\vee} \otimes_{\mathcal{B}}\left(x \otimes_{\mathcal{A}} \Delta\right) & =\left(\Delta^{\vee} \otimes_{\mathcal{B}} \Delta\right) \otimes_{\mathcal{A}} x \\
& =1_{\mathcal{A}} \otimes_{\mathcal{A}} x \\
& =x .
\end{aligned}
$$

As a consequence the two maps (2.22) and (2.24) are inverse to each other, up to the sign given in (2.20) which results from graded commutativity of the exterior product (2.18). (See $[20$, p. 588] and $[32, \S 3]$ for further details.) Thus when $(\mathcal{A}, \mathcal{B})$ is a strong PD pair, the elements $\Delta$ and $\Delta^{\vee}$ establish isomorphisms

$$
\mathrm{K}_{i}(\mathcal{A}) \cong \mathrm{K}^{i+d}(\mathcal{B}) \quad \text { and } \quad \mathrm{K}_{i}(\mathcal{B}) \cong \mathrm{K}^{i-d}(\mathcal{A}),
$$

which is the fundamental property of any form of Poincare duality.

More generally, for any pair of separable $C^{*}$-algebras $(\mathcal{C}, \mathcal{D})$, the maps

$$
\begin{aligned}
\Delta \otimes_{\mathcal{A}}: \mathrm{KK}_{i}(\mathcal{C}, \mathcal{A} \otimes \mathcal{D}) & \longrightarrow \mathrm{KK}_{i+d}(\mathcal{C} \otimes \mathcal{B}, \mathcal{D}), \\
\Delta^{\vee} \otimes_{\mathcal{B}}: \mathrm{KK}_{i}(\mathcal{C}, \mathcal{B} \otimes \mathcal{D}) & \longrightarrow \mathrm{KK}_{i-d}(\mathcal{C} \otimes \mathcal{A}, \mathcal{D})
\end{aligned}
$$

are also isomorphisms, showing that Poincaré duality with arbitrary coefficients holds in this case (Compare [20]). By setting $\mathcal{C}, \mathcal{D}$ equal to various choices from the collection of algebras $\mathbb{C}, \mathcal{A}, \mathcal{B}$, we may infer from (2.27) that the four maps

$$
\begin{aligned}
\Delta \otimes_{\mathcal{A}}: \mathrm{KK}_{i}(\mathcal{A}, \mathcal{A}) & \longrightarrow \mathrm{KK}_{i+d}(\mathcal{A} \otimes \mathcal{B}, \mathbb{C}), \\
\Delta^{\vee} \otimes_{\mathcal{B}}: \mathrm{KK}_{i}(\mathcal{B}, \mathcal{B}) & \longrightarrow \mathrm{KK}_{i-d}(\mathcal{B} \otimes \mathcal{A}, \mathbb{C}), \\
\Delta \otimes_{\mathcal{A}}: \mathrm{KK}_{i}(\mathbb{C}, \mathcal{A} \otimes \mathcal{B}) & \longrightarrow \mathrm{KK}_{i+d}(\mathcal{B}, \mathcal{B}), \\
\Delta^{\vee} \otimes_{\mathcal{B}}: \mathrm{KK}_{i}(\mathbb{C}, \mathcal{B} \otimes \mathcal{A}) & \longrightarrow \mathrm{KK}_{i-d}(\mathcal{A}, \mathcal{A})
\end{aligned}
$$

are all isomorphisms. It follows that if $(\mathcal{A}, \mathcal{B})$ is a strong PD pair, then a fundamental class for $(\mathcal{A}, \mathcal{B})$ induces isomorphisms

$$
\mathrm{K}_{i+d}(\mathcal{A} \otimes \mathcal{B}) \cong \mathrm{KK}_{i}(\mathcal{A}, \mathcal{A}) \cong \mathrm{KK}_{i}(\mathcal{B}, \mathcal{B}) \cong \mathrm{K}^{i+d}(\mathcal{A} \otimes \mathcal{B}) .
$$

Proposition 2.4. Let $\mathcal{A}$ and $\mathcal{B}$ be separable $C^{*}$-algebras. Then:

(1) $\mathcal{A}$ is a strong PD algebra if and only if $\mathcal{A}^{\circ}$ is a strong PD algebra; and 
(2) If $\mathcal{A}$ is Morita equivalent to $\mathcal{B}$, then $\mathcal{A}$ is a strong PD algebra if and only if $\mathcal{B}$ is a strong PD algebra.

Proof. (1) follows easily since $\Delta$ is a fundamental class for $\mathcal{A}$ if and only if it is a fundamental class for $\mathcal{A}^{\mathrm{o}}$, where we identify the KK-groups of $\mathcal{A} \otimes \mathcal{A}^{\mathrm{o}}$ with those of the flip $\mathcal{A}^{\circ} \otimes \mathcal{A}$. The proof of (2) is in [52, §4 Theorem 7].

Example 2.5. Let $X$ be a complete oriented manifold. Then the two pairs of $C^{*}$-algebras $\left(C_{0}(X), C_{0}\left(T^{*} X\right)\right)$ and $\left(C_{0}(X), C_{0}\left(X, \mathrm{Cliff}\left(T^{*} X\right)\right)\right)$ are both strong PD pairs, where Cliff $\left(T^{*} X\right)$ is the Clifford algebra bundle of the cotangent bundle $T^{*} X$. If in addition $X$ is $\operatorname{spin}^{c}$ then $C_{0}\left(\operatorname{Cliff}\left(T^{*} X\right)\right)$ and $C_{0}(X)$ are Morita equivalent, and so $C_{0}(X)$ is a strong PD algebra. If moreover $X$ is compact with boundary $\partial X$ equipped with the induced $\operatorname{spin}^{c}$ structure, then the K-homology connecting homomorphism takes the fundamental class of $X$ to the fundamental class of $\partial X$. It follows that $(C(X), C(X, \partial X))$ is a strong PD pair, where $C(X, \partial X)$ is the $C^{*}$-algebra of continuous functions on $X$ which vanish at the boundary $\partial X$.

Example 2.6. Let $\Gamma$ be a K-amenable, torsion-free discrete group whose classifying space $B \Gamma$ is a smooth oriented manifold. Suppose that $\Gamma$ has the Dirac-dual Dirac property, i.e., for any proper $\Gamma-C^{*}$-algebra $\mathcal{A}$, a Dirac element $\alpha \in \mathrm{KK}_{0}^{\Gamma}(\mathcal{A}, \mathbb{C})$ in the $\Gamma$-equivariant K-homology of $\mathcal{A}$ and a dual Dirac element $\beta \in \mathrm{KK}_{0}^{\Gamma}(\mathbb{C}, \mathcal{A})$ in the $\Gamma$-equivariant K-theory of $\mathcal{A}$ satisfy the conditions

$$
\alpha \otimes_{\mathbb{C}} \beta=1_{\mathcal{A}} \in \mathrm{KK}_{0}^{\Gamma}(\mathcal{A}, \mathcal{A}) \text { and } \beta \otimes_{\mathcal{A}} \alpha=1_{\mathbb{C}} \in \mathrm{KK}_{0}^{\Gamma}(\mathbb{C}, \mathbb{C}) .
$$

The Dirac element $\alpha$ is constructed using a $\operatorname{spin}^{c}$ Dirac operator. Recall that a multiplier $\sigma$ on the group $\Gamma$ is a normalized, $U(1)$-valued group 2-cocycle on $\Gamma$. Its Dixmier-Douady invariant $\delta_{\Gamma}(\sigma) \in \mathrm{H}^{3}(\Gamma, \mathbb{Z})$ is induced in the usual way via the short exact sequence of coefficients in (1.37). Given $\sigma$ we consider the reduced twisted group $C^{*}$-algebra $C_{r}^{*}(\Gamma, \sigma)$. Then $\left(C_{0}\left(T^{*} B \Gamma\right), C_{r}^{*}(\Gamma, \sigma)\right)$ is a strong PD pair for every multiplier $\sigma$ on $\Gamma$ with trivial Dixmier-Douady invariant (we have used the fact that the Baum-Connes conjecture holds for $\Gamma$ in this case). In particular, this holds whenever $\Gamma$ is:

- A torsion-free, discrete subgroup of $S O(n, 1)$ or of $S U(n, 1)$; or

- A torsion-free, amenable group.

If moreover $B \Gamma$ is $\operatorname{spin}^{c}$, then $C_{0}\left(T^{*} B \Gamma\right)$ is a strong PD algebra and hence $C_{r}^{*}(\Gamma, \sigma)$ is a strong PD algebra for every multiplier $\sigma$ on $\Gamma$ with trivial Dixmier-Douady invariant. In particular, we conclude that the noncommutative torus $\mathcal{T}_{\theta}^{2 n}$ is a strong PD algebra, and more generally the noncommutative higher genus Riemann surface $\mathcal{R}_{\theta}^{g}$ is a strong PD algebra. We will consider these latter examples in more detail in Sect. 2.6.

2.4. Duality groups. We will now determine how many fundamental classes a given strong PD pair admits.

Proposition 2.7. Let $(\mathcal{A}, \mathcal{B})$ be a strong PD pair, and let $\Delta \in \mathrm{K}^{d}(\mathcal{A} \otimes \mathcal{B})$ be a fundamental class with inverse $\Delta^{\vee} \in \mathrm{K}_{-d}(\mathcal{A} \otimes \mathcal{B})$. Let $\ell \in \mathrm{KK}_{0}(\mathcal{A}, \mathcal{A})$ be an invertible element. Then $\ell \otimes_{\mathcal{A}} \Delta \in \mathrm{K}^{d}(\mathcal{A} \otimes \mathcal{B})$ is another fundamental class, with inverse $\Delta^{\vee} \otimes_{\mathcal{A}} \ell^{-1} \in \mathrm{K}_{-d}(\mathcal{A} \otimes \mathcal{B})$. 


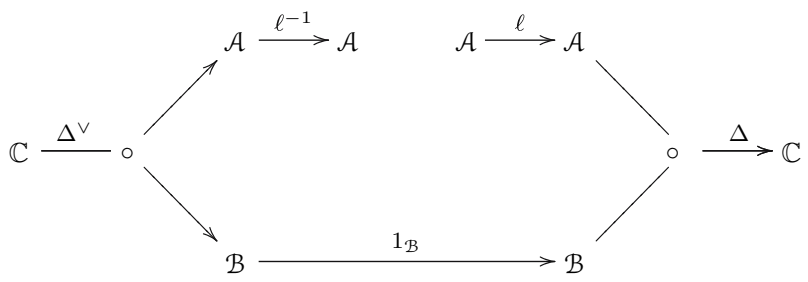

Fig. 2.1. Diagram representing the proof of Proposition 2.7

Proof. Using associativity of the Kasparov product along with the flip isomorphism $\mathcal{A} \otimes \mathcal{B} \rightarrow \mathcal{B} \otimes \mathcal{A}$, we compute

$$
\begin{aligned}
\left(\Delta^{\vee} \otimes_{\mathcal{A}} \ell^{-1}\right) \otimes_{\mathcal{A}}\left(\ell \otimes_{\mathcal{A}} \Delta\right) & =\Delta^{\vee} \otimes_{\mathcal{A}}\left(\ell^{-1} \otimes_{\mathcal{A}} \ell\right) \otimes_{\mathcal{A}} \Delta \\
& =\Delta^{\vee} \otimes_{\mathcal{A}}\left(1_{\mathcal{A}} \otimes_{\mathcal{A}} \Delta\right) \\
& =\Delta^{\vee} \otimes_{\mathcal{A}} \Delta \\
& =(-1)^{d} 1_{\mathcal{B}} .
\end{aligned}
$$

The calculation in the other direction is similar, but slightly trickier because of notational quirks in the way the Kasparov product is written. The calculation goes as follows:

$$
\begin{aligned}
\left(\Delta^{\vee} \otimes_{\mathcal{A}} \ell^{-1}\right) \otimes_{\mathcal{B}}\left(\ell \otimes_{\mathcal{A}} \Delta\right) & =\ell \otimes_{\mathcal{A}}\left(\Delta^{\vee} \otimes_{\mathcal{B}} \Delta\right) \otimes_{\mathcal{A}} \ell^{-1} \\
& =\ell \otimes_{\mathcal{A}} 1_{\mathcal{A}} \otimes_{\mathcal{A}} \ell^{-1} \\
& =\ell \otimes_{\mathcal{A}} \ell^{-1} \\
& =1_{\mathcal{A}} .
\end{aligned}
$$

It would appear that we needed to reorder many of the factors in the product, but in fact, all we are really using is the associativity of the product, in the form discussed in Appendix B. In terms of the diagram calculus discussed there, we simply need to consider the diagram depicted in Fig. 2.1.

This result has a converse.

Proposition 2.8. Let $(\mathcal{A}, \mathcal{B})$ be a strong PD pair, and let $\Delta_{1}, \Delta_{2} \in \mathrm{K}^{d}(\mathcal{A} \otimes \mathcal{B})$ be fundamental classes with inverses $\Delta_{1}^{\vee}, \Delta_{2}^{\vee} \in \mathrm{K}_{-d}(\mathcal{A} \otimes \mathcal{B})$. Then $\Delta_{1}^{\vee} \otimes_{\mathcal{B}} \Delta_{2}$ is an invertible element in $\mathrm{KK}_{0}(\mathcal{A}, \mathcal{A})$, with inverse given by $(-1)^{d} \Delta_{2}^{\vee} \otimes_{\mathcal{B}} \Delta_{1} \in \mathrm{KK}_{0}(\mathcal{A}, \mathcal{A})$.

Proof. As above we compute

$$
\begin{aligned}
\left(\Delta_{1}^{\vee} \otimes_{\mathcal{B}} \Delta_{2}\right) \otimes_{\mathcal{A}}\left(\Delta_{2}^{\vee} \otimes_{\mathcal{B}} \Delta_{1}\right) & =\Delta_{1}^{\vee} \otimes_{\mathcal{B}}\left(\Delta_{2}^{\vee} \otimes_{\mathcal{A}} \Delta_{2}\right) \otimes_{\mathcal{B}} \Delta_{1} \\
& =\Delta_{1}^{\vee} \otimes_{\mathcal{B}}(-1)^{d} 1_{\mathcal{B}} \otimes_{\mathcal{B}} \Delta_{1} \\
& =(-1)^{d} \Delta_{1}^{\vee} \otimes_{\mathcal{B}} \Delta_{1} \\
& =(-1)^{d} 1_{\mathcal{A}},
\end{aligned}
$$

and similarly with $\Delta_{1}$ and $\Delta_{2}$ interchanged.

As an immediate consequence of Propositions 2.7 and 2.8 above, we deduce the following. 
Corollary 2.9. Let $(\mathcal{A}, \mathcal{B})$ be a strong PD pair. Then the moduli space of fundamental classes for $(\mathcal{A}, \mathcal{B})$ is isomorphic to the group of invertible elements in the ring $\mathrm{KK}_{0}(\mathcal{A}, \mathcal{A})$.

We now make some clarifying comments concerning the corollary above. In the physics literature, $\mathrm{K}$-orientations on a smooth manifold $X$ are generally linked to $\operatorname{spin}^{c}$ structures, which (once an orientation has been fixed) are an affine space modeled on the second integral cohomology of $X, \mathrm{H}^{2}(X, \mathbb{Z})$, i.e., the isomorphism classes of line bundles over $X$. However, the space of K-orientations of $X$ is in general much larger, being a principal homogeneous space for the abelian group of units in the ring $\mathrm{K}^{\bullet}(X)$, consisting of stable isomorphism classes of virtual vector bundles over $X$ of virtual rank equal to 1 , and with group operation given by tensor product. The space of all fundamental classes of $X$ in KK-theory is in general still larger, being a principal homogeneous space for the abelian group of units of $\mathrm{KK}_{0}(C(X), C(X))$, which in turn by [80] is an extension of Aut $\mathrm{K}^{\bullet}(X)$ by $\operatorname{Ext}_{\mathbb{Z}}\left(\mathrm{K}^{\bullet}(X), \mathrm{K}^{\bullet+1}(X)\right)$.

Recall that in the situation of Corollary 2.9 there is an isomorphism $\mathrm{KK}_{0}(\mathcal{A}, \mathcal{A}) \cong$ $\mathrm{KK}_{0}(\mathcal{B}, \mathcal{B})$. This moduli space is called the duality group of the pair $(\mathcal{A}, \mathcal{B})$ and is denoted $\mathrm{KK}_{0}(\mathcal{A}, \mathcal{A})^{-1}$. It can be computed explicitly using (2.29) from either the $\mathrm{K}$-theory or the K-homology of the algebra $\mathcal{A} \otimes \mathcal{B}$. We will now describe two illustrative and broad classes of examples of strong PD algebras.

2.5. Spectral triples. There is a natural object that encodes the geometry of a D-brane whose construction can be motivated by the observation that bounded Kasparov modules are not the most useful ones in practical applications of KK-theory, especially when it comes to defining the Kasparov product (2.17) [4]. A zeroth order elliptic pseudodifferential operator $F$ on a smooth closed manifold $X$ determines a class in the K-homology of $X$, i.e., a class in $\mathrm{KK}_{\bullet}(C(X), \mathbb{C})$. However, the product of two such operators need not be a pseudodifferential operator. The Kasparov product is handled better when one uses first order operators instead. But a first order elliptic pseudodifferential operator $D$ will not in general extend to a bounded operator on $L^{2}(X)$ and so will not generally provide a class in $\mathrm{KK}_{\bullet}(C(X), \mathbb{C})$. The trick here, due to Kasparov and reformulated by Baaj and Julg [4], is to replace $D$ by the operator $D\left(1+D^{2}\right)^{-1 / 2}$ which gives rise to a bounded Fredholm module and so produces a class in $\mathrm{KK}_{\bullet}(C(X), \mathbb{C})$. The Baaj-Julg construction generalises to noncommutative $C^{*}$-algebras.

Definition 2.10. A spectral triple over a unital $C^{*}$-algebra $\mathcal{A}$ is a triple $(\mathcal{A}, \mathcal{H}, D)$, where the algebra $\mathcal{A}$ is represented faithfully on a Hilbert space $\mathcal{H}$ and $D$ is an unbounded self-adjoint operator on $\mathcal{H}$ with compact resolvent such that the commutator $[D, a]$ is bounded for all $a \in \mathcal{A}$. In the even case we assume that the Hilbert space $\mathcal{H}$ is $\mathbb{Z}_{2}$-graded and that $D$ is an odd operator with respect to this grading, i.e., there is an involution $\gamma$ on $\mathcal{H}$ which implements the grading and which anticommutes with $D$.

One can prove [4] that all classes in the K-homology of $\mathcal{A}$ are obtained from such spectral triples, which in this context are also called unbounded K-cycles. Together with Connes' axioms [21] such a K-cycle defines a noncommutative $\operatorname{spin}^{c}$ manifold. We will not enter into a detailed account of this latter characterization but refer to [21] and [39, Chap. 10] for a thorough discussion. In the example of a flat D-brane $(W, E, \phi)$ in the spacetime $X$, the spectral triple is $\left(C(X), L^{2}\left(W, \mathrm{~S}_{W} \otimes E\right), D_{E}\right)$ as specified in the proof of Proposition 1.4. This definition can be generalized to provide unbounded $\mathcal{A}-\mathcal{B}$ 
bimodules, which have the property that every element of $\mathrm{KK}_{\bullet}(\mathcal{A}, \mathcal{B})$ arises from such an unbounded bimodule.

Let $(\mathcal{A}, \mathcal{H}, D)$ be an unbounded spectral triple. This fixes our noncommutative spacetime. Let $\mathcal{A}^{0}$ be the opposite algebra of the algebra $\mathcal{A}$. The action of the algebra $\mathcal{A} \otimes \mathcal{A}^{0}$ on the Hilbert space $\mathcal{H}$ is described by using commuting actions of the algebras $\mathcal{A}$ and $\mathcal{A}^{\mathrm{o}}$, making $\mathcal{H}$ into a bimodule over the algebra $\mathcal{A}$. The action of $\mathcal{A}$ is provided by the representation of $\mathcal{A}$ on $\mathcal{H}$ which is part of the data given by the spectral triple $(\mathcal{A}, \mathcal{H}, D)$. The algebra $\mathcal{A}^{\mathrm{o}}$ is assumed to act by means of operators $b^{0}$ for $b \in \mathcal{A}$. We assume that the two actions commute, i.e., $\left[a, b^{\circ}\right]=0$ for all $a, b \in \mathcal{A}$, and that $\left[D, b^{\circ}\right]$ is bounded for all $b \in \mathcal{A}$.

Definition 2.11. The index class of the noncommutative spacetime $(\mathcal{A}, \mathcal{H}, D)$ is the class $\Delta_{D} \in \mathrm{K}^{d}\left(\mathcal{A} \otimes \mathcal{A}^{\mathrm{o}}\right)$ given by the data $\left(\mathcal{A} \otimes \mathcal{A}^{\mathrm{o}}, \mathcal{H}, D\right)$. If the index class is also a fundamental class for $\mathcal{A}$, then its inverse $\Delta_{D}^{\vee} \in \mathrm{K}_{-d}\left(\mathcal{A} \otimes \mathcal{A}^{\circ}\right)$ is called the Bott class of $\mathcal{A}$.

One advantage of the spectral triple formulation is that under suitable circumstances it enables the straightforward construction of a smooth subalgebra $\mathcal{A}^{\infty}$ of a $C^{*}$-algebra $\mathcal{A}$. This is a dense $*$-subalgebra of $\mathcal{A}$ which is stable under holomorphic functional calculus, i.e., if $a \in \mathcal{A}^{\infty}$ with $a=a^{*}$ and $a>0$, then $f(a) \in \mathcal{A}^{\infty}$ for every holomorphic function $f$ on a neighbourhood of the spectrum $\operatorname{Spec}(a)$. By the Karoubi density theorem (see for example [19]), the inclusion homomorphism $\iota: \mathcal{A}^{\infty} \hookrightarrow \mathcal{A}$ then induces an isomorphism in K-theory. Given a spectral triple $(\mathcal{A}, \mathcal{H}, D)$, we assume that the smooth domain

$$
\mathcal{H}^{\infty}=\bigcap_{k \in \mathbb{N}} \operatorname{Dom}\left(D^{k}\right)
$$

of the operator $D$ is an $\mathcal{A}^{\infty}$-bimodule for some smooth subalgebra $\mathcal{A}^{\infty} \subset \mathcal{A}$, or equivalently an $\mathcal{A}^{\infty} \otimes\left(\mathcal{A}^{\infty}\right)^{\mathrm{o}}$-module. We further assume that $\mathcal{A}^{\infty}$ is a Fréchet algebra for the family of semi-norms

$$
q_{k}(a)=\left\|\delta^{k}(a)\right\|
$$

provided by the derivation $\delta: a \mapsto[|D|, a]$ on $\mathcal{A}$. The usage of smooth subalgebras, and in particular topological algebras, will be important later on when we start employing cyclic theory.

Example 2.12. Let $X$ be a compact $\operatorname{spin}^{c}$ manifold of dimension $d$. Let $\mathcal{A}^{\infty}=C^{\infty}(X)$ be the Fréchet algebra of smooth functions on $X$. It acts by pointwise multiplication on the Hilbert space $\mathcal{H}=L^{2}\left(X, \mathrm{~S}_{X}\right)$ of square integrable spinors on $X$. This Hilbert space is $\mathbb{Z}_{2}$-graded when $d$ is even, with the usual grading operator $\gamma$ defining the split $\mathrm{S}_{X}=\mathrm{S}_{X}^{+} \oplus \mathrm{S}_{X}^{-}$into irreducible half-spinor bundles, and ungraded in the odd case. For the operator $D$ we take the usual $\operatorname{spin}^{c}$ Dirac operator $D=\not D$ acting on $\mathcal{H}$. Then $\left(\mathcal{A}^{\infty}, \mathcal{H}, \not D\right)$ defines a cycle $[D D]$ in $\mathrm{K}^{d}(\mathcal{A})$ [39, Theorem 9.20]. This data determines the index class in $\mathrm{K}^{d}\left(\mathcal{A} \otimes \mathcal{A}^{\mathrm{o}}\right)$. Because the algebra $\mathcal{A}$ is commutative in this case, one has $\mathcal{A}=\mathcal{A}^{\mathrm{o}}$ and the multiplication map (2.5) is an algebra homomorphism. Since the $\mathrm{K}$-homology functor is contravariant, there is a map

$$
m^{*}: \mathrm{K}^{d}(\mathcal{A}) \longrightarrow \mathrm{K}^{d}(\mathcal{A} \otimes \mathcal{A})
$$

induced by (2.5). The image of the class $[\not D]$ under this homomorphism is the index class $\Delta_{\not D}[39$, p. 488]. 
2.6. Twisted group algebra completions of surface groups. The noncommutative twotorus $\mathcal{T}_{\theta}^{2}$ provides the original example of noncommutative Poincaré duality which was described by Connes [21] in the context of real spectral triples. It is the specialization to genus one of the example that we present here. Let $\Gamma_{g}$ be the fundamental group of a compact, oriented Riemann surface $\Sigma_{g}$ of genus $g \geq 1$. It has the presentation

$$
\Gamma_{g}=\left\{U_{j}, V_{j}, j=1, \ldots, g \mid \prod_{j=1}^{g}\left[U_{j}, V_{j}\right]=1\right\},
$$

and $B \Gamma_{g}=\Sigma_{g}$ is a smooth spin manifold. Since $\mathrm{H}^{2}\left(\Gamma_{g}, U(1)\right) \cong \mathbb{R} / \mathbb{Z}$, for each $\theta \in[0,1)$ we can identify a unique multiplier $\sigma_{\theta}$ on $\Gamma_{g}$ up to isomorphism. Let $\mathbb{C}\left(\Gamma_{g}, \sigma_{\theta}\right)$ be the $\sigma_{\theta}$-twisted convolution algebra of finitely supported maps $\Gamma_{g} \rightarrow \mathbb{C}$, which is spanned over $\mathbb{C}$ by a set of formal letters $\delta_{\gamma}, \gamma \in \Gamma_{g}$ satisfying $\delta_{\gamma} \delta_{\mu}=\sigma_{\theta}(\gamma, \mu) \delta_{\gamma \mu}$. Let $\|f\|$ denote the operator norm of the operator on $\ell^{2}\left(\Gamma_{g}\right)$ given by left convolution with $f \in \Gamma_{g}$. Then the completion of $\mathbb{C}\left(\Gamma_{g}, \sigma_{\theta}\right)$ with respect to this norm is the reduced twisted group $C^{*}$-algebra $C_{r}^{*}\left(\Gamma_{g}, \sigma_{\theta}\right)$. It can also be viewed as the $C^{*}$-algebra generated by unitaries $U_{j}$ and $V_{j}$ satisfying the commutation relation

$$
\prod_{j=1}^{g}\left[U_{j}, V_{j}\right]=\exp (2 \pi \mathrm{i} \theta) .
$$

On $C_{r}^{*}\left(\Gamma_{g}, \sigma_{\theta}\right)$ there is a canonical trace $\tau$ defined by evaluation at the identity element of $\Gamma_{g}$.

Let $D$ be the operator defined by

$$
D \delta_{\gamma}=\ell(\gamma) \delta_{\gamma}
$$

where $\ell(\gamma) \in[0, \infty)$ is the word length of $\gamma \in \Gamma_{g}$. Let $\delta=\operatorname{ad}(D)$ denote the commutator $[D,-]$. Then $\delta$ is an unbounded closed derivation on the reduced twisted group $C^{*}$-algebra $C_{r}^{*}\left(\Gamma_{g}, \sigma_{\theta}\right)$. Consider the smooth subalgebra

$$
\mathcal{R}^{\infty}\left(\Gamma_{g}, \sigma_{\theta}\right):=\bigcap_{k \in \mathbb{N}} \operatorname{Dom}\left(\delta^{k}\right) .
$$

Since $\mathcal{R}^{\infty}\left(\Gamma_{g}, \sigma_{\theta}\right)$ contains $\delta_{\gamma} \forall \gamma \in \Gamma_{g}$, it contains $\mathbb{C}\left(\Gamma_{g}, \sigma_{\theta}\right)$. Hence it is dense in $C_{r}^{*}\left(\Gamma_{g}, \sigma_{\theta}\right)$. Since $\mathcal{R}^{\infty}\left(\Gamma_{g}, \sigma_{\theta}\right)$ is defined as a domain of derivations, it is closed under holomorphic functional calculus. Because $\Gamma_{g}$ is a surface group, it follows from a variant of a result by Jolissaint [49] that there exists $k \in \mathbb{N}$ and a positive constant $C^{\prime}$ such that for all $f \in \mathbb{C}\left(\Gamma_{g}, \sigma_{\theta}\right)$ one has the Haagerup inequality,

$$
\|f\| \leq C^{\prime} v_{k}(f)
$$

where

$$
v_{k}(f)=\left(\sum_{\gamma \in \Gamma_{g}}(1+\ell(\gamma))^{2 k}|f(\gamma)|^{2}\right)^{1 / 2} .
$$

Using this, it is routine to show that $\mathcal{R}^{\infty}\left(\Gamma_{g}, \sigma_{\theta}\right)$ is a Fréchet algebra, complete in the semi-norms (2.42) induced by $\delta^{k}$. 
To define the fundamental class of the noncommutative Riemann surface $\mathcal{R}_{\theta}^{g}:=$ $\mathcal{R}^{\infty}\left(\Gamma_{g}, \sigma_{\theta}\right)$, we recall from $\S 8$ [16], the K-theory of $C_{r}^{*}\left(\Gamma_{g}, \sigma_{\theta}\right)$. For any $\theta$, the multiplier $\sigma_{\theta}$ has trivial Dixmier-Douady invariant, as $\delta_{\Gamma_{g}}\left(\sigma_{\theta}\right) \in \mathrm{H}^{3}\left(\Sigma_{g}, \mathbb{Z}\right)=0$, and so (via the Baum-Connes assembly map) $\mathrm{K}_{0}\left(C_{r}^{*}\left(\Gamma_{g}, \sigma_{\theta}\right)\right) \cong \mathrm{K}^{0}\left(\Sigma_{g}\right)=\mathbb{Z}^{2}$. For irrational $\theta$, the algebras $C_{r}^{*}\left(\Gamma_{g}, \sigma_{\theta}\right)$ are distinguished for different values of $\theta$ by the image of the trace map induced on K-theory by the trace $\tau$. In a basis $e_{0}, e_{1}$ of $\mathrm{K}_{0}\left(C_{r}^{*}\left(\Gamma_{g}, \sigma_{\theta}\right)\right)$ the trace is given by

$$
\tau\left(n e_{0}+m e_{1}\right)=n+m \theta .
$$

We choose $e_{0}=[1]$, the class of the identity element, and $e_{1}$ such that $\tau\left(e_{1}\right)=\theta$. Another result in $\S 8[16]$ is $\mathrm{K}_{1}\left(C_{r}^{*}\left(\Gamma_{g}, \sigma_{\theta}\right)\right) \cong \mathrm{K}^{1}\left(\Sigma_{g}\right)=\mathbb{Z}^{2 g}$. Moreover, the unitaries $U_{j}$ and $V_{j}$ form a basis for $\mathrm{K}_{1}\left(C_{r}^{*}\left(\Gamma_{g}, \sigma_{\theta}\right)\right)$, 66 [58]. Then the inverse fundamental class of $C_{r}^{*}\left(\Gamma_{g}, \sigma_{\theta}\right)$ is given by

$$
\Delta^{\vee}=e_{0} \otimes e_{1}^{\mathrm{o}}-e_{1} \otimes e_{0}^{\mathrm{o}}+\sum_{j=1}^{g}\left(U_{j} \otimes V_{j}^{\mathrm{o}}-V_{j} \otimes U_{j}^{\mathrm{o}}\right)
$$

The trace $\tau$ also leads to an inner product on $\mathcal{R}_{\theta}^{g}$ defined by $(a, b)=\tau\left(b^{*} a\right)$ for $a, b \in \mathcal{R}_{\theta}^{g}$. Let $L^{2}\left(\mathcal{R}_{\theta}^{g}\right)$ denote the completion of $\mathcal{R}_{\theta}^{g}$ with respect to this inner product, and define $\mathcal{H}:=L^{2}\left(\mathcal{R}_{\theta}^{g}\right) \oplus L^{2}\left(\mathcal{R}_{\theta}^{g}\right)$. Then the element (2.44) is the Bott class of the spectral triple $\left(\mathcal{R}_{\theta}^{g}, \mathcal{H}, D\right)$, with $\mathcal{R}_{\theta}^{g}$ acting diagonally on $\mathcal{H}$ by left multiplication and $D$ odd with respect to the canonical $\mathbb{Z}_{2}$-grading $\gamma$ on $\mathcal{H}$.

2.7. Other notions of Poincaré duality. We now return to the general theory and introduce some alternative weaker forms of the duality described in Sect. 2.3 above, all of which imply the fundamental property (2.26). We start with a "pointwise" version of Definition 2.2.

Definition 2.13 (Weak Poincaré Duality). A pair of separable $C^{*}$-algebras $(\mathcal{A}, \mathcal{B})$ is said to be a weak Poincaré duality pair (weak PD pair for short) if there exists a class $\Delta \in \mathrm{KK}_{d}(\mathcal{A} \otimes \mathcal{B}, \mathbb{C})=\mathrm{K}^{d}(\mathcal{A} \otimes \mathcal{B})$ in the $\mathrm{K}$-homology of $\mathcal{A} \otimes \mathcal{B}$ and a class

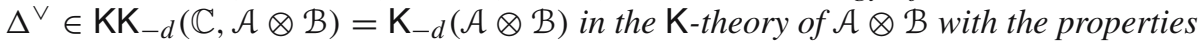

$$
\left(\Delta^{\vee} \otimes_{\mathcal{B}} \Delta\right) \otimes_{\mathcal{A}} x=x \quad \forall x \in \mathrm{KK}_{0}(\mathbb{C}, \mathcal{A})
$$

and

$$
\left(\Delta^{\vee} \otimes_{\mathcal{A}} \Delta\right) \otimes_{\mathcal{B}} y=(-1)^{d} y \quad \forall y \in \mathrm{KK}_{0}(\mathbb{C}, \mathcal{B}) .
$$

A separable $C^{*}$-algebra $\mathcal{A}$ is said to be a weak Poincaré duality algebra (weak PD algebra for short) if $\left(\mathcal{A}, \mathcal{A}^{\mathrm{o}}\right)$ is a weak PD pair.

Example 2.14. Let $\Gamma$ be a torsion-free, discrete group having the Dirac-dual Dirac property such that $B \Gamma$ is a smooth oriented manifold. Then $\left(C_{0}\left(T^{*} B \Gamma\right), C_{r}^{*}(\Gamma, \sigma)\right)$ is a weak PD pair for every multiplier $\sigma$ on $\Gamma$ with trivial Dixmier-Douady invariant. If moreover $B \Gamma$ is $\operatorname{spin}^{c}$, then $C_{0}\left(T^{*} B \Gamma\right)$ is a weak PD algebra and so $C_{r}^{*}(\Gamma, \sigma)$ is a weak PD algebra for every multiplier $\sigma$ on $\Gamma$ with trivial Dixmier-Douady invariant. In particular, this holds whenever $\Gamma$ is: 
- A torsion-free, word hyperbolic group;

- A torsion-free, cocompact lattice in a product of a finite number of groups among Lie or $p$-adic groups of rank one, or in $S L_{3}(\mathbb{F})$ with $\mathbb{F}$ a local field, $\mathbb{H}$ or $E_{6(-26)}$; or

- A torsion-free lattice in a reductive Lie group or in reductive groups over non-archimedean local fields.

The duality of Definition 2.13 can also be weakened to hold only modulo torsion elements of the $\mathrm{K}_{0}$-groups of the algebras involved.

Definition 2.15 (Rational Poincaré Duality). A pair of separable $C^{*}$-algebras $(\mathcal{A}, \mathcal{B})$ is said to be a rational Poincaré duality pair $(\mathbb{Q}-P D$ pair for short) if there exists a class $\Delta \in \mathrm{KK}_{d}(\mathcal{A} \otimes \mathcal{B}, \mathbb{C})=\mathrm{K}^{d}(\mathcal{A} \otimes \mathcal{B})$ in the $\mathrm{K}$-homology of $\mathcal{A} \otimes \mathcal{B}$ and a class

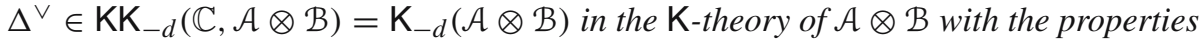

$$
\left(\Delta^{\vee} \otimes_{\mathcal{B}} \Delta\right) \otimes_{\mathcal{A}} x=x \quad \forall x \in \mathrm{KK}_{0}(\mathbb{C}, \mathcal{A}) \otimes \mathbb{Q}
$$

and

$$
\left(\Delta^{\vee} \otimes_{\mathcal{A}} \Delta\right) \otimes_{\mathcal{B}} y=(-1)^{d} y \quad \forall y \in \mathrm{KK}_{0}(\mathbb{C}, \mathcal{B}) \otimes \mathbb{Q} .
$$

A separable $C^{*}$-algebra $\mathcal{A}$ is said to be a rational Poincaré duality algebra $(\mathbb{Q}-P D$ algebra for short) if $\left(\mathcal{A}, \mathcal{A}^{\mathrm{o}}\right)$ is a $\mathbb{Q}-P D$ pair.

Example 2.16. Let $X$ be an oriented rational homology manifold, such as the quotient of a manifold by an orientation-preserving action of a finite group. Then $C_{0}(X)$ is a $\mathbb{Q}-\mathrm{PD}$ algebra.

Example 2.17. Let $\Gamma$ be a discrete group with the Dirac-dual Dirac property and with a torsion-free subgroup $\Gamma_{0}$ of finite index such that $B \Gamma_{0}$ is a smooth oriented manifold. Then $\left(C_{0}\left(T^{*} B \Gamma\right), C_{r}^{*}(\Gamma, \sigma)\right)$ is a $\mathbb{Q}-\mathrm{PD}$ pair for every multiplier $\sigma$ on $\Gamma$. (Note that the Dixmier-Douady invariant in this case is always torsion.) If moreover $B \Gamma_{0}$ is $\operatorname{spin}^{c}$, then $C_{0}\left(T^{*} B \Gamma\right)$ is a $\mathbb{Q}-\mathrm{PD}$ algebra and hence $C_{r}^{*}(\Gamma, \sigma)$ is a $\mathbb{Q}-\mathrm{PD}$ algebra for every multiplier $\sigma$ on $\Gamma$. In particular, this holds whenever $\Gamma$ is:

- A word hyperbolic group; or

- A cocompact lattice in a product of a finite number of groups among Lie or $p$-adic groups of rank one, or in $S L_{3}(\mathbb{F})$ with $\mathbb{F}$ a local field, $\mathbb{H}$ or $E_{6(-26)}$.

Finally, we can take the fundamental property (2.26) itself as the weakest form of the duality.

Definition 2.18 (Poincaré Duality). A pair of separable $C^{*}$-algebras $(\mathcal{A}, \mathcal{B})$ is said to be a Poincaré duality pair (PD pair for short) if there exist isomorphisms

$$
\mathrm{K}_{i}(\mathcal{A}) \cong \mathrm{K}^{i+d}(\mathcal{B}) \quad \text { and } \quad \mathrm{K}_{i}(\mathcal{B}) \cong \mathrm{K}^{i-d}(\mathcal{A}) .
$$

A separable $C^{*}$-algebra $\mathcal{A}$ is said to be a Poincaré duality algebra (PD algebra for short) if $\left(\mathcal{A}, \mathcal{A}^{\circ}\right)$ is a PD pair.

Remark 2.19. For any $C^{*}$-algebra $\mathcal{A}$, the K-theory groups of $\mathcal{A}$ and $\mathcal{A}^{\mathrm{o}}$ are isomorphic. This follows easily from the fact that if $p$ is a projection in $\mathcal{A}$ and $p^{\mathrm{o}}$ is the corresponding element of $\mathcal{A}^{\mathrm{o}}$, then $p^{\mathrm{o}}$ is also a projection. Similarly for a unitary $u \in \mathcal{A}$, the corresponding element $u^{\mathrm{o}}$ of $\mathcal{A}^{\mathrm{o}}$ is also unitary. 
In fact, there is a more general statement, which we will need later. The additive category $\mathrm{KK}$ with separable $C^{*}$-algebras as objects and with $\mathrm{KK}_{\bullet}(\mathcal{A}, \mathcal{B})$ as the morphisms

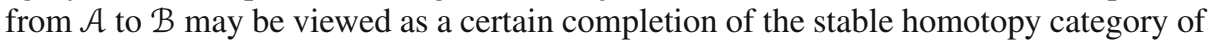
separable $C^{*}$-algebras $[5, \S 22]$. As such, it has an involution ${ }^{\circ}$ induced by the involution $f \mapsto f^{\mathrm{o}}$ sending $f: \mathcal{A} \rightarrow \mathcal{B}$ to the $*$-homomorphism $f^{\mathrm{o}}: \mathcal{A}^{\mathrm{o}} \rightarrow \mathcal{B}^{\mathrm{o}}$ which sends $a^{\mathrm{o}}$ to $(f(a))^{\mathrm{o}}$. The above isomorphism from $\mathrm{K}_{\bullet}(\mathcal{A})$ to $\mathrm{K}_{\bullet}\left(\mathcal{A}^{\mathrm{o}}\right)$ is simply this involution $\mathrm{KK}_{\bullet}(\mathbb{C}, \mathcal{A}) \cong \mathrm{KK}_{\bullet}\left(\mathbb{C}^{\mathrm{o}}=\mathbb{C}, \mathcal{A}^{\mathrm{o}}\right)$.

\section{KK-Equivalence}

In this section we introduce the notion of KK-equivalence and describe its intimate connection to Poincaré duality and Morita equivalence for $C^{*}$-algebras. As in the previous section, we will describe various criteria for the equivalence and describe several natural inequivalent versions of it, giving illustrative commutative and noncommutative examples.

3.1. Strong KK-equivalence. Our first notion of equivalence in KK-theory is a generalization of the standard definition that was essentially already described in Sect. 2.2.

Definition 3.1 (Strong KK-Equivalence). A pair of separable $C^{*}$-algebras $(\mathcal{A}, \mathcal{B})$ are said to be strongly KK-equivalent if there are elements

$$
\alpha \in \mathrm{KK}_{n}(\mathcal{A}, \mathcal{B}) \quad \text { and } \quad \beta \in \mathrm{KK}_{-n}(\mathcal{B}, \mathcal{A})
$$

such that

$$
\alpha \otimes_{\mathcal{B}} \beta=1_{\mathcal{A}} \in \mathrm{KK}_{0}(\mathcal{A}, \mathcal{A}) \quad \text { and } \quad \beta \otimes_{\mathcal{A}} \alpha=1_{\mathcal{B}} \in \mathrm{KK}_{0}(\mathcal{B}, \mathcal{B}) .
$$

The significance of this definition stems from the following results.

Lemma 3.2. Suppose that the pair of separable $C^{*}$-algebras $(\mathcal{A}, \mathcal{B})$ are strongly $\mathrm{KK}$ equivalent. Then the maps

$$
\begin{array}{ll}
\alpha \otimes_{\mathcal{B}}: \mathrm{K}^{i}(\mathcal{B}) \longrightarrow \mathrm{K}^{i+n}(\mathcal{A}), & \beta \otimes_{\mathcal{A}}: \mathrm{K}^{i}(\mathcal{A}) \longrightarrow \mathrm{K}^{i-n}(\mathcal{B}), \\
\otimes_{\mathcal{B}} \alpha: \mathrm{K}_{i}(\mathcal{B}) \longrightarrow \mathrm{K}_{i+n}(\mathcal{A}), & \otimes_{\mathcal{A}} \beta: \mathrm{K}_{i}(\mathcal{A}) \longrightarrow \mathrm{K}_{i-n}(\mathcal{B})
\end{array}
$$

are all isomorphisms.

Proof. By the associativity property of the Kasparov product, the maps in (3.3) satisfy

$$
\begin{aligned}
\beta \otimes_{\mathcal{A}}\left(\alpha \otimes_{\mathcal{B}} x\right) & =\left(\beta \otimes_{\mathcal{A}} \alpha\right) \otimes_{\mathcal{B}} x \\
& =1_{\mathcal{B}} \otimes_{\mathcal{B}} x \\
& =x \quad \forall x \in \mathrm{K}^{i}(\mathcal{B}), \\
\alpha \otimes_{\mathcal{B}}\left(\beta \otimes_{\mathcal{A}} y\right) & =\left(\alpha \otimes_{\mathcal{B}} \beta\right) \otimes_{\mathcal{A}} y \\
& =1_{\mathcal{A}} \otimes_{\mathcal{A}} y \\
& =y \quad \forall y \in \mathrm{K}^{i}(\mathcal{A}),
\end{aligned}
$$


and are therefore all isomorphisms. Again by the associativity property of the Kasparov product, the maps in (3.4) satisfy

$$
\begin{aligned}
\left(z \otimes_{\mathcal{B}} \alpha\right) \otimes_{\mathcal{A}} \beta & =z \otimes_{\mathcal{B}}\left(\alpha \otimes_{\mathcal{A}} \beta\right) \\
& =z \otimes_{\mathcal{B}} 1_{\mathcal{B}} \\
& =z \quad \forall z \in \mathrm{K}_{i}(\mathcal{B}), \\
\left(w \otimes_{\mathcal{A}} \beta\right) \otimes_{\mathcal{B}} \alpha & =w \otimes_{\mathcal{A}}\left(\beta \otimes_{\mathcal{B}} \alpha\right) \\
& =w \otimes_{\mathcal{A}} 1_{\mathcal{A}} \\
& =w \quad \forall w \in \mathrm{K}_{i}(\mathcal{A}),
\end{aligned}
$$

and thus are also isomorphisms.

Remark 3.3. As in (2.27), one can generalize the isomorphisms of Lemma 3.2 above to arbitrary coefficients. For any pair of separable $C^{*}$-algebras $(\mathcal{C}, \mathcal{D})$, the maps

$$
\begin{array}{r}
\alpha \otimes_{\mathcal{B}}: \mathrm{KK}_{i}(\mathcal{C} \otimes \mathcal{B}, \mathcal{D}) \longrightarrow \mathrm{KK}_{i+n}(\mathcal{C} \otimes \mathcal{A}, \mathcal{D}), \\
\beta \otimes_{\mathcal{A}}: \mathrm{KK}_{i}(\mathcal{C} \otimes \mathcal{A}, \mathcal{D}) \longrightarrow \mathrm{KK}_{i-n}(\mathcal{C} \otimes \mathcal{B}, \mathcal{D}), \\
\otimes_{\mathcal{B}} \alpha: \mathrm{KK}_{i}(\mathcal{C}, \mathcal{B} \otimes \mathcal{D}) \longrightarrow \mathrm{KK}_{i+n}(\mathcal{C}, \mathcal{A} \otimes \mathcal{D}), \\
\otimes_{\mathcal{A}} \beta: \mathrm{KK}_{i}(\mathcal{C}, \mathcal{A} \otimes \mathcal{D}) \longrightarrow \mathrm{KK}_{i-n}(\mathcal{C}, \mathcal{B} \otimes \mathcal{D})
\end{array}
$$

are all isomorphisms.

Lemma 3.4. Suppose that the pair of separable $C^{*}$-algebras $(\mathcal{A}, \mathcal{B})$ are strongly $\mathrm{KK}$ equivalent. Then $\mathcal{A}$ is a PD algebra (resp., strong PD algebra, weak PD algebra) if and only if $\mathcal{B}$ is a PD algebra (resp., strong PD algebra, weak PD algebra).

Proof. If $\mathcal{A}$ is a $\mathrm{PD}$ algebra, then there are isomorphisms $\mathrm{K}_{i}(\mathcal{A}) \cong \mathrm{K}^{i+d}(\mathcal{A})$. Combining this with the isomorphisms given in Lemma 3.2, we deduce that there are isomorphisms $\mathrm{K}_{i}(\mathcal{B}) \cong \mathrm{K}^{i+d}(\mathcal{B})$, showing that $\mathcal{B}$ is also a $\mathrm{PD}$ algebra. The argument is symmetric, proving the result.

We will now investigate some circumstances under which KK-equivalence holds. Let $\mathcal{A}$ be a $C^{*}$-algebra, and let $\mathcal{H}$ be a Hilbert $\mathcal{A}$-module. Recall from Sect. 2.2 that the norm closure of the linear span of the set $\left\{\left\langle\zeta \mid \zeta^{\prime}\right\rangle \mid \zeta, \zeta^{\prime} \in \mathcal{H}\right\}$ is the algebra $\mathcal{K}(\mathcal{H})$ of compact operators on $\mathcal{H}$. The module $\mathcal{H}$ is said to be full if $\mathcal{K}(\mathcal{H})$ is equal to $\mathcal{A}$.

Definition 3.5 (Strong Morita equivalence). Two $C^{*}$-algebras $\mathcal{A}$ and $\mathcal{B}$ are said to be strongly Morita equivalent if there is a full Hilbert $\mathcal{A}$-module $\mathcal{H}$ such that $\mathcal{K}(\mathcal{H}) \cong \mathcal{B}$.

Upon identifying $\mathcal{B}$ with $\mathcal{K}(\mathcal{H})$, we define a Hilbert $(\mathcal{A}, \mathcal{B})$-bimodule $\mathcal{H}^{\vee}$ as follows. As sets (or real vector spaces), one has $\mathcal{H}^{\vee}=\mathcal{H}$. Let $\zeta \mapsto \zeta^{\vee}$ denote the identity map on $\mathcal{H} \rightarrow \mathcal{H}^{\vee}$. Since $\lambda \zeta^{\vee}=(\bar{\lambda} \zeta)^{\vee}$ for $\lambda \in \mathbb{C}$ and $\zeta \in \mathcal{H}$, it follows that the identity map is conjugate linear. For $\zeta_{1}, \zeta_{2} \in \mathcal{H}^{\vee}, a \in \mathcal{A}$ and $b \in \mathcal{B}$ we set

$$
a \zeta_{1}^{\vee}=\left(\zeta_{1} a^{*}\right)^{\vee}, \quad \zeta_{1}^{\vee} b=\left(b^{*} \zeta_{1}\right)^{\vee}, \quad \text { and }\left\langle\zeta_{1}^{\vee}, \zeta_{2}^{\vee}\right\rangle=\zeta_{1}\left\langle\zeta_{2},-\right\rangle
$$

Then $\mathcal{H}^{\vee}$ is a Hilbert $\mathcal{B}$-module which is full by definition. Moreover, the map $\zeta_{1}^{\vee}\left\langle\zeta_{2}^{\vee},-\right\rangle$ $\mapsto\left\langle\zeta_{1}, \zeta_{2}\right\rangle$ identifies $\mathcal{K}\left(\mathcal{H}^{\vee}\right)$ with $\mathcal{A}$. From the point of view of the present paper, the importance of this notion stems from the fact that Morita equivalent algebras encode the same physics. The following well-known lemma relates strong Morita equivalence to strong KK-equivalence. 
Lemma 3.6. Let $\mathcal{A}$ and $\mathcal{B}$ be separable $C^{*}$-algebras. If $\mathcal{A}$ is strongly Morita equivalent to $\mathcal{B}$, then $\mathcal{A}$ is strongly $\mathrm{KK}$-equivalent to $\mathcal{B}$.

Proof. Let $\mathcal{H}$ be a full Hilbert $\mathcal{A}$-module implementing the Morita equivalence between $\mathcal{A}$ and $\mathcal{B}$. Define elements $\beta \in \mathrm{KK}_{0}(\mathcal{B}, \mathcal{A})$ by the equivalence class of $(\mathcal{H}, i, 0)$, where $i$ : $\mathcal{B} \rightarrow \mathcal{K}(\mathcal{H})$ is the identity, and $\alpha \in \mathrm{KK}_{0}(\mathcal{A}, \mathcal{B})$ by the equivalence class of $\left(\mathcal{H}^{\vee}, i^{\vee}, 0\right)$ in the notation above. Then the map $\zeta_{1} \otimes \zeta_{2}^{\vee} \mapsto \zeta_{1}\left\langle\zeta_{2},-\right\rangle$ identifies the $\mathcal{B}$-bimodule $\mathcal{H} \otimes_{\mathcal{A}} \mathcal{H}^{\vee}$ with $\mathcal{B}$, and hence $\beta \otimes_{\mathcal{A}} \alpha=1_{\mathcal{B}}$. Similarly, the map $\zeta_{1}^{\vee} \otimes \zeta_{2} \mapsto \zeta_{1}^{\vee}\left\langle\zeta_{2}^{\vee},-\right\rangle$ identifies the $\mathcal{A}$-bimodule $\mathcal{H}^{\vee} \otimes_{\mathcal{B}} \mathcal{H}$ with $\mathcal{A}$. Therefore $\alpha \otimes_{\mathcal{B}} \beta=1_{\mathcal{A}}$, proving that $(\mathcal{A}, \mathcal{B})$ is a strongly $\mathrm{KK}$-equivalent pair.

There are many examples of strongly KK-equivalent algebras that are not strongly Morita equivalent. For example, by [80] any two type I separable $C^{*}$-algebras with the same $\mathrm{K}_{0}$ and $\mathrm{K}_{1}$ groups are automatically strongly $\mathrm{KK}$-equivalent. Another famous example concerns the two-dimensional noncommutative tori $\mathcal{T}_{\theta}^{2}$. We recall $[68,77]$ that $\mathcal{T}_{\theta}^{2}$ is Morita equivalent to $\mathcal{T}_{\theta^{\prime}}^{2}$ if and only if $\theta$ and $\theta^{\prime}$ belong to the same $G L_{2}(\mathbb{Z})$ orbit. On the other hand, the algebras $\mathcal{T}_{\theta}^{2}$ and $C\left(\mathbb{T}^{2}\right)$ are strongly KK-equivalent for all $\theta$ [68].

The following lemma from [52] gives us more examples of strongly KK-equivalent algebras.

Lemma 3.7. The Thom isomorphism for an oriented real vector bundle $E \rightarrow X$ gives a natural strong $\mathrm{KK}$-equivalence between the algebra $C_{0}(E)$ of continuous functions on $E$ vanishing at infinity and the algebra $C_{0}(X, \mathrm{Cliff}(E))$ of continuous sections, vanishing at infinity, of the Clifford algebra bundle $\mathrm{Cliff}(E)$ of $E$.

Remark 3.8. If $E \rightarrow X$ is a $\operatorname{spin}^{c}$ vector bundle then $\delta_{X}(\operatorname{Cliff}(E))=0$, and the $C^{*}$-algebras $C_{0}(\mathrm{Cliff}(E))$ and $C_{0}(X)$ are strongly Morita equivalent. In this case the space of sections $C_{0}(X, \operatorname{Cliff}(E))$ can be replaced by $C_{0}(X)$ in Lemma 3.7.

3.2. Other notions of KK-equivalence. We now introduce variants of the concept of strong KK-equivalence.

Definition 3.9 (Weak KK-Equivalence). A pair of separable $C^{*}$-algebras $(\mathcal{A}, \mathcal{B})$ are said to be weakly KK-equivalent if there are elements

$$
\alpha \in \mathrm{KK}_{n}(\mathcal{A}, \mathcal{B}) \quad \text { and } \quad \beta \in \mathrm{KK}_{-n}(\mathcal{B}, \mathcal{A})
$$

such that

$$
\left(\alpha \otimes_{\mathcal{B}} \beta\right) \otimes_{\mathcal{A}} y=y \quad \forall y \in \mathrm{K}^{i}(\mathcal{A}), \quad z \otimes_{\mathcal{B}}\left(\alpha \otimes_{\mathcal{A}} \beta\right)=z \quad \forall z \in \mathrm{K}_{i}(\mathcal{B})
$$

and

$$
\left(\beta \otimes_{\mathcal{A}} \alpha\right) \otimes_{\mathcal{B}} x=x \quad \forall x \in \mathrm{K}^{i}(\mathcal{B}), \quad w \otimes_{\mathcal{A}}\left(\beta \otimes_{\mathcal{B}} \alpha\right)=w \quad \forall w \in \mathrm{K}_{i}(\mathcal{A})
$$

Definition 3.10 (Rational KK-Equivalence). A pair of separable $C^{*}$-algebras $(\mathcal{A}, \mathcal{B})$ are said to be rationally KK-equivalent if there are elements

$$
\alpha \in \mathrm{KK}_{n}(\mathcal{A}, \mathcal{B}) \quad \text { and } \quad \beta \in \mathrm{KK}_{-n}(\mathcal{B}, \mathcal{A})
$$


such that

$$
\left(\alpha \otimes_{\mathcal{B}} \beta\right) \otimes_{\mathcal{A}} y=y \quad \forall y \in \mathrm{K}^{i}(\mathcal{A}) \otimes \mathbb{Q}, \quad z \otimes_{\mathcal{B}}\left(\alpha \otimes_{\mathcal{A}} \beta\right)=z \quad \forall z \in \mathrm{K}_{i}(\mathcal{B}) \otimes \mathbb{Q}
$$

and

$\left(\beta \otimes_{\mathcal{A}} \alpha\right) \otimes_{\mathcal{B}} x=x \quad \forall x \in \mathrm{K}^{i}(\mathcal{B}) \otimes \mathbb{Q}, w \otimes_{\mathcal{A}}\left(\beta \otimes_{\mathcal{B}} \alpha\right)=w \quad \forall w \in \mathrm{K}_{i}(\mathcal{A}) \otimes \mathbb{Q}$.

Definition 3.11 (K-Equivalence). A pair of separable $C^{*}$-algebras $(\mathcal{A}, \mathcal{B})$ are said to be K-equivalent if there are isomorphisms

$$
\mathrm{K}_{i}(\mathcal{A}) \cong \mathrm{K}_{i-n}(\mathcal{B}) \quad \text { and } \quad \mathrm{K}^{i}(\mathcal{A}) \cong \mathrm{K}^{i-n}(\mathcal{B}) .
$$

With a proof along the lines of Lemma 3.4, one can prove the following.

Lemma 3.12. Suppose that the pair of separable $C^{*}$-algebras $(\mathcal{A}, \mathcal{B})$ are weakly $\mathrm{KK}$ equivalent (resp., rationally $\mathrm{KK}$-equivalent, K-equivalent). Then $\mathcal{A}$ is a weak PD algebra (resp., $\mathbb{Q}-P D$ algebra, PD algebra) if and only if $\mathcal{B}$ is a weak PD algebra (resp., $\mathbb{Q}-P D$ algebra, PD algebra).

In the remainder of this section we will give some classes of examples illustrating the various notions of KK-equivalence introduced above.

3.3. Universal coefficient theorem. To understand the relation between weak and strong KK-equivalence, we appeal to the universal coefficient theorem of Rosenberg and Schochet [80]. It holds precisely for the class $\mathfrak{N}$ of $C^{*}$-algebras which are KK-equivalent to commutative $C^{*}$-algebras. For every pair of $C^{*}$-algebras $(\mathcal{A}, \mathcal{B})$ with $\mathcal{A} \in \underline{\mathfrak{N}}$ and $\mathcal{B}$ separable, there is a split short exact sequence of abelian groups given by

$$
0 \rightarrow \operatorname{Ext}_{\mathbb{Z}}\left(\mathrm{K}_{\bullet+1}(\mathcal{A}), \mathrm{K}_{\bullet}(\mathcal{B})\right) \rightarrow \mathrm{KK}_{\bullet}(\mathcal{A}, \mathcal{B}) \rightarrow \operatorname{Hom}_{\mathbb{Z}}\left(\mathrm{K}_{\bullet}(\mathcal{A}), \mathrm{K}_{\bullet}(\mathcal{B})\right) \rightarrow 0 .
$$

Since there are many examples of $C^{*}$-algebras which are not in $\underline{\mathfrak{N}}$ [83], the notion of K-equivalence may strictly contain that of strong KK-equivalence. More precisely, suppose that $\mathcal{A}$ is not in $\underline{\mathfrak{R}}$. If $\mathcal{A}$ satisfies the universal coefficient theorem for one-variable $\mathrm{K}$-homology but not for KK-theory, then $\mathcal{A}$ is $\mathrm{K}$-equivalent to a commutative $C^{*}$-algebra but not strongly KK-equivalent to such an algebra. (We do not know if such algebras exist, but this is a possibility.)

Remark 3.13. From Remark 2.19 and the universal coefficient theorem (3.16) it follows that if $\mathcal{A}$ lies in the category $\mathfrak{N}$ of $C^{*}$-algebras discussed above, then $\mathcal{A}$ and $\mathcal{A}^{0}$ are strongly KK-equivalent. (It is easy to construct examples, however, where they are not Morita equivalent.) We are not sure if $\mathcal{A}$ and $\mathcal{A}^{\mathrm{o}}$ are always strongly KK-equivalent, without any hypotheses on $\mathcal{A}$. 
3.4. Deformations. Let $\mathcal{A}$ and $\mathcal{B}$ be $C^{*}$-algebras. A deformation of $\mathcal{A}$ into $\mathcal{B}$ is a continuous field of $C^{*}$-algebras over a half-open interval $[0, \varepsilon)$, locally trivial over the open interval $(0, \varepsilon)$, whose fibre over 0 is $\mathcal{A}$ and whose fibres over $\hbar \in(0, \varepsilon)$ are all isomorphic to $\mathcal{B}$. A deformation gives rise to an extension of $C^{*}$-algebras

$$
0 \longrightarrow C_{0}((0, \varepsilon), \mathcal{B}) \longrightarrow \mathcal{C} \longrightarrow \mathcal{A} \longrightarrow 0 .
$$

Connes and Higson observed that any deformation from $\mathcal{A}$ into $\mathcal{B}$ defines a morphism $\mathrm{K}_{\bullet}(\mathcal{A}) \rightarrow \mathrm{K}_{\bullet}(\mathcal{B})$, which is simply the connecting homomorphism $\partial$ in the six-term exact K-theory sequence associated to (3.17). Moreover, when $\mathcal{A}$ is nuclear, the extension (3.17) has a completely positive cross-section and thus defines an element in $\mathrm{KK}_{0}(\mathcal{A}, \mathcal{B})$ which induces the map on K-theory groups.

3.5. Homotopy equivalence. Let $\mathcal{A}$ and $\mathcal{B}$ be $C^{*}$-algebras. Two algebra homomorphisms $\phi_{0}, \phi_{1}: \mathcal{A} \rightarrow \mathcal{B}$ are homotopic if there is a path $\gamma_{t}, t \in[0,1]$ of homomorphisms $\gamma_{t}: \mathcal{A} \rightarrow \mathcal{B}$ such that $t \mapsto \gamma_{t}(a)$ is a norm continuous path in $\mathcal{B}$ for every $a \in \mathcal{A}$ and such that $\gamma_{0}=\phi_{0}, \gamma_{1}=\phi_{1}$. The algebras $\mathcal{A}$ and $\mathcal{B}$ are said to be homotopy equivalent if there exist morphisms $\phi: \mathcal{A} \rightarrow \mathcal{B}$ and $\eta: \mathcal{B} \rightarrow \mathcal{A}$ whose compositions $\eta \circ \phi$ and $\phi \circ \eta$ are homotopic to the identity maps on $\mathcal{A}$ and $\mathcal{B}$, respectively. The algebra $\mathcal{A}$ is called contractible if it is homotopy equivalent to the zero algebra.

Lemma 3.14. If $\mathcal{A}$ and $\mathcal{B}$ are homotopy equivalent $C^{*}$-algebras, then the pair $(\mathcal{A}, \mathcal{B})$ are strongly $\mathrm{KK}$-equivalent.

Proof. Suppose that $\phi: \mathcal{A} \rightarrow \mathcal{B}$ and $\eta: \mathcal{B} \rightarrow \mathcal{A}$ are $*$-homomorphisms which are homotopy inverses to one another. Then they define classes $\mathrm{KK}(\phi) \in \mathrm{KK}_{0}(\mathcal{A}, \mathcal{B})$ and $\mathrm{KK}(\eta) \in \mathrm{KK}_{0}(\mathcal{B}, \mathcal{A})$ whose $\mathrm{Kasparov}$ products are simply $\mathrm{KK}(\eta \circ \phi) \in \mathrm{KK}_{0}(\mathcal{A}, \mathcal{A})$ and $\mathrm{KK}(\phi \circ \eta) \in \mathrm{KK}_{0}(\mathcal{B}, \mathcal{B})$. Since $\eta \circ \phi$ is homotopic to $\mathrm{id}_{\mathcal{A}}$, one has $\mathrm{KK}(\eta \circ \phi)=1_{\mathcal{A}}$ by homotopy invariance of KK-theory, and similarly $\mathrm{KK}(\phi \circ \eta)=1_{\mathcal{B}}$.

\section{Cyclic Theory}

As was crucial in the definition of D-brane charge given in Sect. 1.1, the topological K-theory of a spacetime $X$ is connected to its cohomology through the rational isomorphism provided by the Chern character ch : $\mathrm{K}^{\bullet}(X) \otimes \mathbb{Q} \rightarrow \mathrm{H}^{\bullet}(X, \mathbb{Q})$. While this works well in the case of flat D-branes, in the more general settings described before we need a more general cohomological framework in which to express the D-brane charge, particularly when the branes are described by a noncommutative algebra $\mathcal{A}$. The appropriate receptacle for the Chern character in analytic K-theory is a suitable version of the cyclic cohomology of the given algebra $\mathcal{A}$. In this section we will present an overview of the general aspects of cyclic homology and cohomology. As we will see later on, this general formulation provides a nice intrinsic definition of the D-brane charge even in the flat commutative case, which moreover has a suitable extension to the noncommutative situations.

4.1. Formal properties of cyclic homology theories. Cyclic cohomology of a complex algebra, from its introduction by Connes, was developed in parallel with K-theory as a noncommutative analogue of the de Rham cohomology of a differentiable manifold. One 
of the main features of that theory, which made it a very useful tool to aid in computations of K-theory, is the existence of the Chern character from K-theory of the algebra to the cyclic cohomology of the algebra. Compared to K-theory, cyclic type homology theories exhibit a major weakness: they are all defined using a suitably chosen deformation of the tensor algebra. In the case of a topological algebra $\mathcal{A}$, there are many ways to topologize the tensor algebra $T \mathcal{A}$ : this makes cyclic type homology theories very sensitive to the choice of topology.

In this section we introduce the properties that we shall require of the cohomology theory to make it suitable for our purposes. We shall then briefly outline the main points in the construction of cyclic type homology theories that will satisfy those properties.

Let $\mathcal{A}$ and $\mathcal{B}$ be topological algebras, whose topology will be specified shortly. We shall denote by $\mathrm{HL}_{i}(\mathcal{A}, \mathcal{B}), i=0,1$, a bivariant cyclic theory associated with $\mathcal{A}$ and $\mathcal{B}$ that has the following formal properties:

(1) $\mathrm{HL}_{i}(\mathcal{A}, \mathcal{B})$ is covariant in the second variable and contravariant in the first variable;

(2) For any three algebras $\mathcal{A}, \mathcal{B}$ and $\mathcal{C}$ there is a natural composition product

$$
\otimes_{\mathcal{B}}: \mathrm{HL}_{i}(\mathcal{A}, \mathcal{B}) \times \mathrm{HL}_{j}(\mathcal{B}, \mathcal{C}) \rightarrow \mathrm{HL}_{i+j}(\mathcal{A}, \mathcal{C})
$$

(3) The functor $\mathrm{HL}_{i}(-,-)$ is split exact and satisfies excision in each variable;

(4) $\mathrm{HL}_{i}(-,-)$ is homotopy invariant;

(5) For any algebras $\mathcal{A}_{1}, \mathcal{A}_{2}, \mathcal{B}_{1}$, and $\mathcal{B}_{2}$, there is an exterior product

$$
\mathrm{HL}_{i}\left(\mathcal{A}_{1}, \mathcal{B}_{1}\right) \times \mathrm{HL}_{j}\left(\mathcal{A}_{2}, \mathcal{B}_{2}\right) \rightarrow \mathrm{HL}_{i+j}\left(\mathcal{A}_{1} \otimes \mathcal{A}_{2}, \mathcal{B}_{1} \otimes \mathcal{B}_{2}\right)
$$

compatible with the composition product; and

(6) When $\mathcal{A}$ and $\mathcal{B}$ are $C^{*}$-algebras, there is a natural transformation of functors, the Chern character,

$$
\operatorname{ch}: \mathrm{KK}_{i}(\mathcal{A}, \mathcal{B}) \rightarrow \mathrm{HL}_{i}(\mathcal{A}, \mathcal{B})
$$

which is compatible with the product on $\mathrm{KK}$ and the composition product on HL.

Often we suppress the subscript $i$ when $i=0$. Axiom (2) ensures that $H_{\bullet}(\mathcal{A}, \mathcal{A})$ is a $\mathbb{Z}_{2}$-graded ring. There are now many definitions of bivariant cyclic theories of this kind, each suited to a specific category of algebras. (See [73] for a survey of these theories, as well as the relationships among them.) With every choice of a class of algebras we need to specify the notions of homotopy and stability which are suitable for the given category. In many cases, for example when $\mathcal{A}$ and $\mathcal{B}$ are multiplicatively convex ( $m$-convex) algebras, KK in property (6) needs to be replaced by a different form of bivariant K-theory, for example, Cuntz's $k k$ [26], which is defined on a class of $m$-convex algebras. Cuntz's $k k$ is much easier to define than KK, but it is harder to compute, and at present, the precise relation between $k k$ and $\mathrm{KK}$ is unclear. This is why we are led to consider Puschnigg's local bivariant cyclic cohomology, developed primarily in [73], which we shall denote $\mathrm{HL}_{i}(-,-){ }^{1}$ This theory, which can be defined on a class of $C^{*}$-algebras which is suitable for our purposes, is closest to Kasparov's KK-theory.

Furthermore we need to point out that the correct notion of tensor product in property (5) depends on the theory. When working with nuclear $C^{*}$-algebras, the usual $C^{*}$-tensor product is appropriate, but when working with Fréchet algebras, one might need the projective tensor product. When we do not assume that $\mathcal{A}$ and $\mathcal{B}$ are topological algebras,

\footnotetext{
1 Puschnigg calls it $\mathrm{HC}^{\text {loc }}$ or $\mathrm{HE}^{\text {loc }}$, but as this is a bit cumbersome, we have chosen to simplify the notation.
} 
then the natural cyclic type homology theory to consider is the bivariant periodic cyclic homology $\operatorname{HP}_{i}(\mathcal{A}, \mathcal{B})$ of Cuntz and Quillen [30]. This theory is closest to the cyclic homology and cohomology defined by Connes. We have that $\operatorname{HP}_{i}(\mathcal{A}, \mathbb{C})$ is the same as the periodic cyclic cohomology of $\mathcal{A}, \operatorname{HP}^{i}(\mathcal{A})$, while $\mathrm{HP}_{i}(\mathbb{C}, \mathcal{A})$ coincides with the periodic cyclic homology, $\operatorname{HP}_{i}(\mathcal{A})$.

When the algebra $\mathcal{A}$ is equipped with a topology, Meyer's work indicates [62] that in the construction of a reasonable cyclic type theory one should consider bounded rather than continuous maps. More precisely, this means the following. As is well known, a map of topological vector spaces $f: E \rightarrow F$ is bounded if and only if it sends bounded sets in $E$ to bounded sets in $F$. If $E$ and $F$ are locally convex, then a reasonable definition of a bounded set states that a subset of $E$ is bounded if and only if it is absorbed by every open neighbourhood of zero. Since the choice of open neighbourhoods in this definition is dictated by topology, the class of bounded sets in $E$ is fixed by the choice of topology. The class of bounded sets in a topological space is called a bornology $\mathfrak{B}$; the bornology associated with the topology of a space is called the von Neumann bornology. A space equipped with a chosen family of bounded sets is called a bornological space. A bornology on a vector space $E$ is a class $\mathfrak{B}$ of subsets of $E$, which have the properties that $\{e\} \in \mathfrak{B}$ for all $e \in E$; if $S \in \mathfrak{B}$ and $T \subset S$, then $T \in \mathfrak{B}$ (a subset of a bounded set is bounded); if $S, T \in \mathfrak{B}$, then $S \cup T \in \mathfrak{B}$ (the union of two bounded sets is bounded); $c \cdot S \in \mathfrak{B}$ if $S \in \mathfrak{B}$ and $c \in \mathbb{C}$ (a scalar multiple of a bounded set is bounded); and if $S, T \in \mathfrak{B}$ then $S+T \in \mathfrak{B}$ (vector addition in $E$ is a bounded map). A vector space equipped with a bornology is called a bornological vector space. A map $f: E \rightarrow F$ of bornological spaces is called bounded if and only if it sends elements of the bornology in $E$ (i.e., the 'bounded' sets in $E$ ) to elements of the bornology in $F$.

In the study of bornological spaces it became clear that it is useful to consider the choice of bornology to be independent from the choice of topology. This observation lies at the basis of Meyer's construction of his analytic cyclic homology $\operatorname{HA}_{i}(-,-)$, which is a bivariant functor defined on a class of bornological algebras. A bornological vector space $\mathcal{A}$ is a bornological algebra if and only if it is equipped with a multiplication $m: \mathcal{A} \times \mathcal{A} \rightarrow \mathcal{A}$ which is bounded in the bornological sense. Meyer's analytic theory is very flexible and can be used in a variety of contexts. For example, it contains the Cuntz-Quillen bivariant cyclic theory $\operatorname{HP}_{i}(\mathcal{A}, \mathcal{B})$ of [30]. Moreover, it can be defined for Fréchet algebras and, in particular, for Banach algebras. Meyer showed in his thesis that for a suitable choice of bornology on a locally convex algebra $\mathcal{A}$ his analytic cyclic cohomology $\mathrm{HA}_{i}(\mathcal{A}, \mathbb{C})=\mathrm{HA}^{i}(\mathcal{A})$ is isomorphic to Connes' entire cyclic cohomology $\mathrm{HE}^{i}(\mathcal{A})[62$, Thm. 3.47]. A very important example of an entire cyclic cohomology class is given by the JLO cocycle, which provides the character of a $\theta$-summable Fredholm module [47].

4.2. Local cyclic theory. We shall now outline, in broad terms, the construction and main properties of Puschnigg's local cyclic theory. For any algebra $\mathcal{A}$, unital or not, the (non-unital) tensor algebra $T \mathcal{A}$ of $\mathcal{A}$ is defined by

$$
T \mathcal{A}=\mathcal{A} \oplus(\mathcal{A} \otimes \mathcal{A}) \oplus(\mathcal{A} \otimes \mathcal{A} \otimes \mathcal{A}) \oplus \ldots
$$

In applications, $\mathcal{A}$ will be assumed to be complete with respect to some additional structure. For example, $\mathcal{A}$ may be a Fréchet or Banach algebra, or in the bornological case, $\mathcal{A}$ will be assumed to be a complete bornological algebra $[62,2.2]$. In each case the definition of $T \mathcal{A}$ will require a choice of a completed tensor product which is relevant 
to the given situation. For example, for Banach algebras a reasonable choice is the completed projective tensor product $\hat{\otimes}_{\pi}$ while for bornological algebras we need to take the completed bornological tensor product $[62,2.2 .3]$. The tensor algebra is closely related to the algebra of noncommutative differential forms $\Omega \mathcal{A}$, which is defined (as a vector space) by

$$
\Omega^{n} \mathcal{A}=\mathcal{A}^{\otimes n+1} \oplus \mathcal{A}^{\otimes n}, \quad n>0 .
$$

We put $\Omega^{0} \mathcal{A}=\mathcal{A} .^{2}$ One defines a differential d on $\Omega \mathcal{A}$ of degree +1 , which for $n \geq 1$ is given by the two-by-two matrix

$$
\mathrm{d}=\left(\begin{array}{ll}
0 & 0 \\
1 & 0
\end{array}\right),
$$

while in degree zero we put $\mathrm{d}=(0,1): \mathcal{A} \rightarrow \mathcal{A}^{\otimes 2} \oplus \mathcal{A}$. One can then show that, for $n>0, \Omega^{n} \mathcal{A} \cong \operatorname{Span}\left\{\tilde{a}_{0} \mathrm{~d} a_{1} \ldots \mathrm{d} a_{n}\right\}$, where $\tilde{a}_{0}$ is an element of the unitization $\tilde{\mathcal{A}}$ of $\mathcal{A}$, and $a_{i} \in \mathcal{A}$. The multiplication on $\Omega \mathcal{A}$ is uniquely determined by the requirements that $\mathrm{d}$ be a derivation (satisfying the Leibnitz rule) and that

$$
\begin{aligned}
& \left(\mathrm{d} a_{1} \ldots \mathrm{d} a_{n}\right) \cdot\left(\mathrm{d} a_{n+1} \ldots \mathrm{d} a_{n+m}\right)=\mathrm{d} a_{1} \ldots \mathrm{d} a_{n+m}, \\
& \left(\tilde{a}_{0} \mathrm{~d} a_{1} \ldots \mathrm{d} a_{n}\right) \cdot\left(\mathrm{d} a_{n+1} \ldots \mathrm{d} a_{n+m}\right)=\tilde{a}_{0} \mathrm{~d} a_{1} \ldots \mathrm{d} a_{n+m} .
\end{aligned}
$$

A key point in the construction of any cyclic type homology theory is the choice of a suitable completion (depending on whether $\mathcal{A}$ is considered to be a topological or a bornological algebra) of $\Omega \mathcal{A}$. To retain the important universal property of the tensor algebra, this completion is also usefully described as a deformation of the tensor algebra denoted $X(T \mathcal{A})$. This is a $\mathbb{Z}_{2}$ graded complex defined very simply as follows:

$$
\Omega^{1}(T \mathcal{A}) /\left[\Omega^{1}(T \mathcal{A}), \Omega^{1}(T \mathcal{A})\right] \stackrel{\mathrm{b}}{\rightarrow} \Omega^{0}(T \mathcal{A}),
$$

where $\left[\Omega^{1}(T \mathcal{A}), \Omega^{1}(T \mathcal{A})\right]$, the commutator space of $\Omega^{1}(T \mathcal{A})$, is spanned by the set of all commutators $[\omega, \eta]$ with $\omega, \eta \in \Omega^{1}(T \mathcal{A})$. The map b is given by $\omega_{0} \mathrm{~d} \omega_{1} \mapsto\left[\omega_{0}, \omega_{1}\right]$ for any two $\omega_{0}, \omega_{1} \in T \mathcal{A}$. There is a differential going the other way, which is the composition of the differential $\mathrm{d}: T \mathcal{A} \rightarrow \Omega^{1}(T \mathcal{A})$ with the quotient map $\Omega^{1}(T \mathcal{A}) \rightarrow$ $\Omega^{1}(T \mathcal{A}) /\left[\Omega^{1}(T \mathcal{A}), \Omega^{1}(T \mathcal{A})\right]$.

Let $\mathcal{A}$ and $\mathcal{B}$ be two complete bornological algebras and let $X(T \mathcal{A})^{c}$ be the Puschnigg completion of the $X(T \mathcal{A})$ (see [72, §5], [28, §23]). There is a $\mathbb{Z}_{2}$-graded complex of bounded maps $\operatorname{Hom}_{\mathbb{C}}\left(X(T \mathcal{A})^{c}, X(T \mathcal{B})^{c}\right)$. We define the bivariant local cyclic homology by

$$
\mathrm{HL}_{i}(\mathcal{A}, \mathcal{B})=\mathrm{H}_{i}\left(\operatorname{Hom}_{\mathbb{C}}\left(X(T \mathcal{A})^{c}, X(T \mathcal{B})^{c}\right)\right)
$$

where $i=0,1$ [28]. This homology theory coincides with other theories discussed there under suitable conditions. For example, when $\mathcal{B}$ is a Fréchet algebra whose bornology is specified by the family of pre-compact sets (or is nuclear) then $\mathrm{HA}_{\bullet}(\mathcal{B})=\mathrm{HL}_{\bullet}(\mathcal{B})$ and there is a natural map $\mathrm{HA}_{\bullet}(\mathcal{A}, \mathcal{B}) \rightarrow \mathrm{HL}_{\bullet}(\mathcal{A}, \mathcal{B})$.

We recall the notion of a smooth subalgebra of a complete bornological algebra.

\footnotetext{
${ }^{2}$ Caution: In [73], Puschnigg forgets to mention this, i.e., to mention that the definition of $\Omega^{n} \mathcal{A}$ is different when $n=0$.
} 
Definition 4.1. [28, 23.3]. Let $\mathcal{A}$ be a complete bornological algebra with bornology $\mathfrak{B}(\mathcal{A})$, which is a dense subalgebra of a Banach algebra $\mathcal{B}$ with closed unit ball $U$. Then $\mathcal{A}$ is a smooth subalgebra of $\mathcal{B}$ if and only if for every element $S \in \mathfrak{B}(\mathcal{A})$ such that $S \subset r U$, for some $r<1$, the set $S^{\infty}=\bigcup_{n} S^{n}$ is an element of $\mathfrak{B}(\mathcal{A})$.

Smooth subalgebras of Banach algebras are closed under the holomorphic functional calculus. The following result will be important to us.

Theorem 4.2. [28, 23.4]. Let $\mathcal{B}$ be a Banach algebra with the metric approximation property. Let $\mathcal{A}$ be a smooth subalgebra of $\mathcal{B}$. Then $\mathcal{A}$ and $\mathcal{B}$ are $\mathrm{HL}$-equivalent, that is the inclusion map $\mathcal{A} \rightarrow \mathcal{B}$ induces an invertible element of $\mathrm{HL}_{0}(\mathcal{A}, \mathcal{B})$.

Note by [17] that all nuclear $C^{*}$-algebras have the metric approximation property. Some, but not all, non-nuclear $C^{*}$-algebras have it as well.

Example 4.3. Let $X$ be a compact manifold. Then the Fréchet algebra $C^{\infty}(X)$ is a smooth subalgebra of the algebra $C(X)$ of continuous functions on $X$. Furthermore, the inclusion $C^{\infty}(X) \hookrightarrow C(X)$ is an invertible element in $\mathrm{HL}\left(C^{\infty}(X), C(X)\right)$ by Theorem 4.2 above. In particular, both the local homology and cohomology of these two algebras are isomorphic. Puschnigg also proves that $\mathrm{HL}_{\bullet}\left(C^{\infty}(X)\right) \cong \mathrm{HP}_{\bullet}\left(C^{\infty}(X)\right)$, and so, in this case, Puschnigg's local cyclic theory coincides with the standard periodic cyclic homology. The following fundamental result of Connes makes it possible to establish contact between Puschnigg's local cyclic theory of $C(X)$ and the de Rham cohomology of $X$.

Theorem 4.4. For $X$ a compact manifold, the periodic cyclic homology $\mathrm{HP}_{\bullet}\left(C^{\infty}(X)\right)$ is canonically isomorphic to the periodic de Rham cohomology:

$$
\operatorname{HP}_{0}\left(C^{\infty}(X)\right) \cong \mathrm{H}_{\mathrm{dR}}^{\text {even }}(X) \quad \text { and } \quad \mathrm{HP}_{1}\left(C^{\infty}(X)\right) \cong \mathrm{H}_{\mathrm{dR}}^{\text {odd }}(X)
$$

It is in the sense of this theorem that we may regard cyclic homology as a generalization of de Rham cohomology to other (possibly noncommutative) settings.

The local cyclic theory HL admits a Chern character with the required properties.

Theorem 4.5. $[28,23.5]$. Let $\mathcal{A}$ and $\mathcal{B}$ be separable $C^{*}$-algebras. Then there exists a natural bivariant Chern character

$$
\operatorname{ch}: \mathrm{KK}_{\bullet}(\mathcal{A}, \mathcal{B}) \rightarrow \mathrm{HL}_{\bullet}(\mathcal{A}, \mathcal{B})
$$

which has the following properties:

(1) ch is multiplicative, i.e., if $\alpha \in \mathrm{KK}_{i}(\mathcal{A}, \mathcal{B})$ and $\beta \in \mathrm{KK}_{j}(\mathcal{B}$, $\mathcal{C})$ then

$$
\operatorname{ch}\left(\alpha \otimes_{\mathcal{B}} \beta\right)=\operatorname{ch}(\alpha) \otimes_{\mathcal{B}} \operatorname{ch}(\beta) ;
$$

(2) ch is compatible with the exterior product; and

(3) $\operatorname{ch}(\mathrm{KK}(\phi))=\mathrm{HL}(\phi)$ for any algebra homomorphism $\phi: \mathcal{A} \rightarrow \mathcal{B}$.

The last property implies that the Chern character sends invertible elements of KK-theory to invertible elements of bivariant local cyclic cohomology.

Moreover, if $\mathcal{A}$ and $\mathcal{B}$ are in the class $\underline{\mathfrak{N}}$ of $C^{*}$-algebras for which the Universal Coefficient Theorem holds in $\mathrm{KK}$-theory, then

$$
\mathrm{HL}_{\bullet}(\mathcal{A}, \mathcal{B}) \cong \operatorname{Hom}_{\mathbb{C}}\left(\mathrm{K}_{\bullet}(\mathcal{A}) \otimes_{\mathbb{Z}} \mathbb{C}, \mathrm{K}_{\bullet}(\mathcal{B}) \otimes_{\mathbb{Z}} \mathbb{C}\right) .
$$

If $\mathrm{K}_{\bullet}(\mathcal{A})$ is finitely generated, this is also equal to $\mathrm{KK}_{\bullet}(\mathcal{A}, \mathcal{B}) \otimes_{\mathbb{Z}} \mathbb{C}$. 


\section{Duality in Bivariant Cyclic Cohomology}

In this section we shall formulate and analyse Poincaré duality in the context of bivariant cyclic cohomology of generic noncommutative algebras. Our analysis of Poincaré duality in KK-theory from Sect. 2 and of KK-equivalence in Sect. 3 indicates that it is possible to define analogous notions in any bivariant theory that has the same formal properties as KK-theory. An important example of such a situation is provided by the bivariant local cyclic theory as introduced in Sect. 4.2, where we have the additional tool of the bivariant Chern character from KK. Rather than repeating all the details, we shall simply state the main points. Duality in cyclic homology and periodic cyclic homology has also been considered by Gorokhovsky [38, §5.2].

5.1. Poincaré duality. We will now develop the periodic cyclic theory analogues of the versions of Poincaré duality introduced in Sect. 2. Because we want to link everything with $\mathrm{KK}$ and not with $k k$ or its variants, we will work throughout with $\mathrm{HL}$ and not with $\mathrm{HP}$, even though the latter is probably more familiar to most readers.

Definition 5.1. Two complete bornological algebras $\mathcal{A}, \mathcal{B}$ are a strong cyclic Poincaré dual pair (strong $C$-PD pair for short) if there exists a class $\Xi \in \mathrm{HL}_{d}(\mathcal{A} \otimes \mathcal{B}, \mathbb{C})=$ $\mathrm{HL}_{d}(\mathcal{A} \otimes \mathcal{B})$ in the local cyclic cohomology of $\mathcal{A} \otimes \mathcal{B}$ and a class $\Xi^{\vee} \in \mathrm{HL}_{d}(\mathbb{C}, \mathcal{A} \otimes \mathcal{B})=$ $\mathrm{HL}_{d}(\mathcal{A} \otimes \mathcal{B})$ in the local cyclic homology of $\mathcal{A} \otimes \mathcal{B}$ with the properties

$$
\Xi^{\vee} \otimes_{\mathcal{B}} \Xi=1_{\mathcal{A}} \in \mathrm{HL}_{0}(\mathcal{A}, \mathcal{A}) \text { and } \Xi^{\vee} \otimes_{\mathcal{A}} \Xi=(-1)^{d} 1_{\mathcal{B}} \in \mathrm{HL}_{0}(\mathcal{B}, \mathcal{B}) \text {. }
$$

The class $\Xi$ is called a fundamental cyclic cohomology class for the pair $(\mathcal{A}, \mathcal{B})$ and $\Xi^{\vee}$ is called its inverse. A complete bornological algebra $\mathcal{A}$ is a strong cyclic Poincaré duality algebra ( strong $\boldsymbol{C}$-PD algebra for short) if $\left(\mathcal{A}, \mathcal{A}^{\circ}\right)$ is a strong $C$-PD pair.

As in the case of KK-theory, these hypotheses establish an isomorphism between the periodic cyclic homology and cohomology of the algebras $\mathcal{A}$ and $\mathcal{B}$ as

$$
\mathrm{HL}_{\bullet}(\mathcal{A}) \cong \mathrm{HL}^{\bullet+d}(\mathcal{B}) \quad \text { and } \quad \mathrm{HL}_{\bullet}(\mathcal{B}) \cong \mathrm{HL}^{\bullet+d}(\mathcal{A}) \text {. }
$$

One also has the isomorphisms

$$
\mathrm{HL}_{\bullet+d}(\mathcal{A} \otimes \mathcal{B}) \cong \mathrm{HL}_{\bullet}(\mathcal{A}, \mathcal{A}) \cong \mathrm{HL}_{\bullet}(\mathcal{B}, \mathcal{B}) \cong \mathrm{HL}^{\bullet+d}(\mathcal{A} \otimes \mathcal{B})
$$

The moduli space of fundamental cyclic cohomology classes for the pair $(\mathcal{A}, \mathcal{B})$ is the cyclic duality group $\operatorname{HL}_{0}(\mathcal{A}, \mathcal{A})^{-1}$ of invertible elements of the $\operatorname{ring} \operatorname{HL}_{0}(\mathcal{A}, \mathcal{A}) \cong$ $\mathrm{HL}_{0}(\mathcal{B}, \mathcal{B})$. Similarly to Sect. 2.7 , one has the alternative notions of weak C-PD pairs and of cyclic Poincaré duality.

Example 5.2. Let $\mathcal{A}=C^{\infty}(X)$ be the algebra of smooth functions on a compact oriented manifold $X$ of dimension $d$. Then the image of the class $\left[\varphi_{X}\right]$ of the cyclic $d$-cocycle

$$
\varphi_{X}\left(f^{0}, f^{1}, \ldots, f^{d}\right)=\frac{1}{d !} \int_{X} f^{0} \mathrm{~d} f^{1} \wedge \cdots \wedge \mathrm{d} f^{d}
$$

for $f^{i} \in \mathcal{A}$, under the homomorphism $m^{*}: \mathrm{HP}^{\bullet}(\mathcal{A}) \cong \mathrm{HL}^{\bullet}(C(X)) \rightarrow \mathrm{HL}^{\bullet}(C(X) \otimes$ $C(X))$ induced by the product map (2.5), is the fundamental class $\Xi \in \mathrm{HL}^{d}(C(X) \otimes$ $C(X))$ of $X$ in cyclic cohomology. Thus in this case $\Xi$ corresponds to the orientation 
cycle $[X]$ and our notion of Poincaré duality agrees with the classical one. More generally, if $X$ is non-orientable, i.e., $\mathrm{w}_{1}(X) \neq 0$, we choose the local coefficient system $\mathrm{C}_{X} \rightarrow X$ associated to $\mathrm{w}_{1}(X)$ whose fibres are each (non-canonically) isomorphic to $\mathbb{Z}$. Then $\left(C(X), C\left(X, \mathrm{C}_{X} \otimes \mathbb{C}\right)\right)$ is a strong C-PD pair.

Thanks to the existence of a universal, multiplicative Chern character which maps the bivariant KK-theory to bivariant local cyclic cohomology, we can show that Definitions 2.20 and 5.1 are compatible. Let $(\mathcal{A}, \mathcal{B})$ be a strong PD pair of algebras in KK-theory with fundamental class $\Delta \in \mathrm{K}^{d}(\mathcal{A} \otimes \mathcal{B})$ and inverse $\Delta^{\vee} \in \mathrm{K}_{-d}(\mathcal{A} \otimes \mathcal{B})$. Then there is a commutative diagram

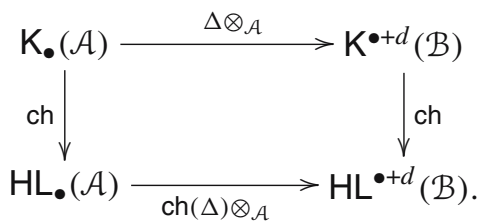

Since the Chern character is a unital homomorphism the cocycle $\operatorname{ch}(\Delta)$ is an invertible class in $\mathrm{HL}^{d}(\mathcal{A} \otimes \mathcal{B})$ with inverse $\operatorname{ch}\left(\Delta^{\vee}\right) \in \mathrm{HL}_{d}(\mathcal{A} \otimes \mathcal{B})$, and so it establishes Poincaré duality in local cyclic cohomology, i.e., Poincaré duality in KK-theory implies Poincaré duality in cyclic theory. However, the converse is not true, since the cyclic theories constructed in Sect. 4 give vector spaces over $\mathbb{C}$ and are thus insensitive to torsion. A simple example is provided by any compact oriented manifold $X$ for which $\mathrm{W}_{3}(X) \neq 0$. Then the algebra $\mathcal{A}=C(X)$ is a strong C-PD algebra but not a PD algebra. In the cases where the Chern characters $\mathrm{ch}_{\mathcal{A}}$ and $\mathrm{ch}_{\mathcal{B}}$ are both isomorphisms after tensoring the K-groups with $\mathbb{C}$, Poincaré duality in cyclic theory implies rational Poincaré duality in K-theory.

The commutative diagram (5.4) allows us to transport the structure of Poincaré duality in KK-theory to local cyclic cohomology. In particular, all examples that we presented in Sect. 2 in the context of KK-theory also apply to local cyclic cohomology. Note, however, that if a strong $\mathrm{PD}$ pair of algebras $(\mathcal{A}, \mathcal{B})$ are equipped with their own fundamental cyclic cohomology class $\Xi \in \mathrm{HL}^{d}(\mathcal{A} \otimes \mathcal{B})$, then generically $\operatorname{ch}(\Delta) \neq \Xi$. We will see an example of this in Sect. 5.3 below. In fact, this will be the crux of our construction of D-brane charge cycles in Sect. 8 . The choice $\operatorname{ch}(\Delta)=\Xi$ has certain special properties which will be discussed in Sect. 7.1.

5.2. HL-Equivalence. Exactly as we did above, it is possible to define the analogous notion of KK-equivalence from Sect. 3 in the bivariant local cyclic cohomology. We now briefly discuss how this works.

Definition 5.3. Two complete bornological algebras $\mathcal{A}$ and $\mathcal{B}$ are said to be strongly $\mathrm{HL}-$ equivalent if there are elements

$$
\xi \in \mathrm{HL}_{n}(\mathcal{A}, \mathcal{B}) \quad \text { and } \quad \eta \in \mathrm{HL}_{n}(\mathcal{B}, \mathcal{A})
$$

such that

$$
\xi \otimes_{\mathcal{B}} \eta=1_{\mathcal{A}} \in \mathrm{HL}_{0}(\mathcal{A}, \mathcal{A}) \quad \text { and } \quad \eta \otimes_{\mathcal{A}} \xi=1_{\mathcal{B}} \in \mathrm{HL}_{0}(\mathcal{B}, \mathcal{B})
$$


As in the case of KK-theory, these hypotheses induce isomorphisms between the local cyclic homology and cohomology groups of the algebras $\mathcal{A}$ and $\mathcal{B}$ as

$$
\mathrm{HL}^{\bullet}(\mathcal{A}) \cong \mathrm{HL}^{\bullet+n}(\mathcal{B}) \quad \text { and } \quad \mathrm{HL}_{\bullet}(\mathcal{A}) \cong \mathrm{HL}_{\bullet+n}(\mathcal{B}) .
$$

One similarly has the notions of weak equivalence and of HL-equivalence. The Chern character again allows us to transport results of Sect. 3 to cyclic theory. If $(\mathcal{A}, \mathcal{B})$ is a pair of strongly KK-equivalent algebras, with the equivalence implemented by classes $\alpha \in \mathrm{KK}_{n}(\mathcal{A}, \mathcal{B})$ and $\beta \in \mathrm{KK}_{-n}(\mathcal{B}, \mathcal{A})$, then there are commutative diagrams

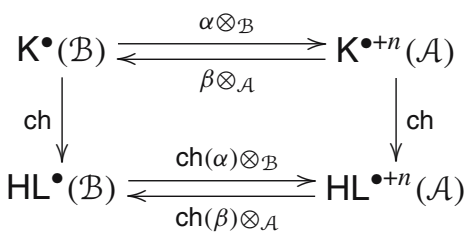

and

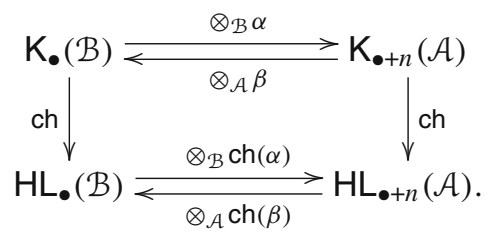

Thus KK-equivalence implies HL-equivalence, but not conversely.

Remark 5.4. In the various versions of cyclic theory, one needs different notions of Morita equivalence. For example, one has an isomorphism $\mathrm{HP}_{\bullet}(\mathcal{A}) \cong \mathrm{HP}_{\bullet}\left(\mathcal{A} \widehat{\otimes} \mathcal{L}^{1}\right)$ in periodic cyclic homology, where $\mathcal{L}^{1}$ is the algebra of trace-class operators on a separable Hilbert space. Fortunately, since $\mathrm{HL}$ is well behaved for $C^{*}$-algebras, which are our main examples of interest, we will usually not have to worry about this point.

5.3. Spectral triples. Let us model a D-brane by an even spectral triple $(\mathcal{A}, \mathcal{H}, D)$ as prescribed in Sect. 2.5. Assume that the resolvent of the operator $D$ is of order $p$, i.e., its eigenvalues $\mu_{k}$ decay as $k^{-1 / p}$. This is the situation, for example, for the case when $D$ is the canonical Dirac operator over a finite-dimensional $\operatorname{spin}^{c}$ manifold. We further make the following regularity assumption on the spectral triple. Let us assume that both $\mathcal{A}$ and $[D, \mathcal{A}]$ are contained in $\bigcap_{k>0} \operatorname{Dom}\left(\delta^{k}\right)$, where $\delta=\operatorname{ad}(|D|)$. Let $\Sigma \subset \mathbb{C}$ denote the set of all singularities of the spectral zeta-functions $\zeta_{P}(z)=\operatorname{Tr}_{\mathcal{H}}\left(P|D|^{-z}\right)$, where $z \in \mathbb{C}$ and $P$ is an element of the algebra generated by $\delta^{k}(\mathcal{A})$ and $\delta^{k}([D, \mathcal{A}])$. The set $\Sigma$ is called the dimension spectrum of the spectral triple $(\mathcal{A}, \mathcal{H}, D)$. We will assume that $(\mathcal{A}, \mathcal{H}, D)$ has discrete and simple dimension spectrum $\Sigma$, i.e., that $\zeta_{P}, \forall P$ can be extended as a meromorphic function to $\mathbb{C} \backslash \Sigma$ with simple poles in $\Sigma$. Such a spectral triple is said to be regular.

Under these circumstances, the residue formula

$$
f P=\operatorname{Res}_{z=0}\left(\operatorname{Tr}_{\mathcal{H}}\left(P|D|^{-2 z}\right)\right)
$$


defines a trace on the algebra generated by $\mathcal{A},[D, \mathcal{A}]$ and $|D|^{z}, z \in \mathbb{C}$. Using it we may define the Connes-Moscovici cocycle $\varphi^{\mathrm{CM}}=\left(\varphi_{2 n}^{\mathrm{CM}}\right)_{n \geq 0}$ in the (b, B) bi-complex of the algebra $\mathcal{A}$ [25]. For this, we denote by $a^{[k]}$ the $k^{\text {th }}$ iterated commutator of $a \in \mathcal{A}$ with the operator $D^{2}$,

$$
a^{[k]}:=\underbrace{\left[D^{2},\left[D^{2}, \cdots\left[D^{2}\right.\right.\right.}_{k \text { times }}, a]] \cdots] .
$$

For $n=0$ and $a_{0} \in \mathcal{A}$ we set

$$
\varphi_{0}^{\mathrm{CM}}\left(a_{0}\right)=\operatorname{Tr}_{\mathcal{H}}\left(\gamma a_{0} \Pi_{\mathrm{ker}(D)}\right)+\operatorname{Res}_{z=0}\left(\frac{1}{z} \operatorname{Tr}_{\mathcal{H}}\left(\gamma a_{0}|D|^{-2 z}\right)\right),
$$

where $\Pi_{\operatorname{ker}(D)}$ is the orthogonal projection onto the kernel of the operator $D$ on $\mathcal{H}$. For $n>0$ and $a_{i} \in \mathcal{A}$, we define

$$
\begin{aligned}
& \varphi_{2 n}^{\mathrm{CM}}\left(a_{0}, a_{1}, \ldots, a_{2 n}\right) \\
& =\sum_{\vec{k}} \frac{(-1)^{|\vec{k}|}(|\vec{k}|+n-1) !}{2 \vec{k} ! \prod_{j=1}^{2 n}\left(k_{1}+\cdots+k_{j}+j\right)} f \gamma a_{0}\left(\prod_{j=1}^{2 n}\left[D, a_{j}\right]^{\left[k_{j}\right]}\right)|D|^{-2|\vec{k}|-2 n},
\end{aligned}
$$

where the sum runs through all multi-indices $\vec{k}=\left(k_{1}, \ldots, k_{2 n}\right)$ with $|\vec{k}|:=k_{1}+\cdots+k_{2 n}$ and $\vec{k} !:=k_{1} ! \cdots k_{2 n} !$. It can be shown that this formula has only a finite number of nonzero terms. The class $\operatorname{ch}(D)=\left[\varphi_{2 n}^{\mathrm{CM}}\right] \in \operatorname{HE}^{0}(\mathcal{A})$ is called the (even) cyclic cohomology Chern character and it may be regarded as a map

$$
\operatorname{ch}: \mathrm{K}^{0}(\mathcal{A}) \longrightarrow \mathrm{HE}^{0}(\mathcal{A}) \text {. }
$$

It is instructive again to look at the case where $X$ is a compact, smooth spin manifold of even dimension $d$. For the spectral triple we then take $\mathcal{A}=C^{\infty}(X), \mathcal{H}^{ \pm}=L^{2}\left(X, \mathrm{~S}_{X}^{ \pm}\right)$, and $D=\not D: C^{\infty}\left(X, \mathrm{~S}_{X}^{+}\right) \rightarrow C^{\infty}\left(X, \mathrm{~S}_{X}^{-}\right)$, the usual (untwisted) Dirac operator. Then the dimension spectrum $\Sigma$ consists of relative integers $<d$, and is simple. (Multiplicities would arise in the case that the spacetime $X$ is a singular orbifold, for example.) We can thereby apply the Connes-Moscovici cocycle construction to this situation. One finds that the contributions to (5.14) vanish unless $\vec{k}=\overrightarrow{0}$, and hence its components are given explicitly by [70]

$$
\varphi_{2 n}^{\mathrm{CM}}\left(f^{0}, f^{1}, \ldots, f^{2 n}\right)=\frac{1}{(2 n) !} \int_{X} f^{0} \mathrm{~d} f^{1} \wedge \cdots \wedge \mathrm{d} f^{2 n} \wedge \widehat{A}(X)
$$

with $f^{i} \in C^{\infty}(X)$. In this case, the entire cyclic cohomology $\mathrm{HE}^{\bullet}(\mathcal{A})$ is naturally isomorphic to the local cyclic cohomology $\mathrm{HL}^{\bullet}(\mathcal{A})$ and to the periodic cyclic cohomology $\operatorname{HP}^{\bullet}(\mathcal{A})[72]$; the resulting entire cocycle is cohomologous to an explicit periodic cyclic cocycle given in terms of the spectral triple.

This result implies an important characterization that will be crucial to the construction of brane charges as cyclic classes. 
Theorem 5.5. Let $X$ be a compact, smooth spin manifold of even dimension. Then the cyclic cohomology Chern character of the spectral triple $\left(C^{\infty}(X), L^{2}\left(X, \mathrm{~S}_{X}\right), \not D\right)$ coincides with the Atiyah-Hirzebruch class of $X$ in even de Rham homology,

$$
\operatorname{ch}(\not D)=\operatorname{Pd}_{X}^{-1}(\widehat{A}(X)) \text {. }
$$

Remark 5.6. In some cases the $p$-summability requirements are not met, most notably when the spectral triple is infinite-dimensional. In such instances we can still compute the cyclic cohomology Chern character if the spectral triple $(\mathcal{A}, \mathcal{H}, D)$ is $\theta$-summable, i.e., $[D, a]$ is bounded for all $a \in \mathcal{A}$, and the eigenvalues $\mu_{k}$ of the resolvent of $D$ grow no faster than $\log (k)$. This implies that the corresponding heat kernel is trace-class, $\operatorname{Tr}_{\mathcal{H}}\left(\mathrm{e}^{-t D^{2}}\right)<\infty \forall t>0$. Within this framework, we can then represent the Chern character of the even spectral triple $(\mathcal{A}, \mathcal{H}, D)$ in the entire cyclic cohomology of $\mathcal{A}$ by using the $J L O$ cocycle $\varphi^{\mathrm{JLO}}=\left(\varphi_{2 n}^{\mathrm{JLO}}\right)_{n \geq 0}$ [47]. With $a_{0}, a_{1} \ldots, a_{2 n} \in \mathcal{A}$, it is defined by the formula

$$
\begin{aligned}
& \varphi_{2 n}^{\mathrm{JLO}}\left(a_{0}, a_{1} \ldots, a_{2 n}\right) \\
& =\int_{\Delta_{2 n}} \mathrm{~d} t_{0} \mathrm{~d} t_{1} \cdots \mathrm{d} t_{2 n} \operatorname{Tr}_{\mathcal{H}}\left(\gamma a_{0} \mathrm{e}^{-t_{0} D^{2}}\left(\prod_{j=1}^{2 n}\left[D, a_{j}\right] \mathrm{e}^{-t_{j} D^{2}}\right)\right),
\end{aligned}
$$

where $\triangle_{n}=\left\{\left(t_{0}, t_{1}, \ldots, t_{n}\right) \mid t_{i} \geq 0, \quad \sum_{i} t_{i}=1\right\}$ denotes the standard $n$-simplex in $\mathbb{R}^{n+1}$. This entire cyclic cocycle is cohomologous to the Chern character. Once again, consider the example of the canonical triple $\left(C^{\infty}(X), L^{2}\left(X, \mathrm{~S}_{X}\right), \not D\right)$ over a spin manifold $X$, and replace $\not D$ everywhere in the formula (5.17) by $s \not D$ with $s>0$. By using asymptotic symbol calculus, one can then show $[24,74]$ that the character $(5.17)$ retracts as $s \rightarrow 0$ to the Connes-Moscovici cocycle (5.15).

\section{T-Duality}

In this section we will show that there is a strong link between KK-equivalence for crossed product algebras and T-duality in string theory. This will lead to a putative axiomatic characterization of T-duality for $C^{*}$-algebras. We also describe an analogous characterization in local cyclic cohomology.

6.1. Duality for crossed products. We begin with some general results regarding KKequivalence and Poincaré duality for crossed product algebras, and then use them to give some more examples of PD algebras. Let $G$ be a locally compact, connected Lie group. Recall [18] that $G$ is said to satisfy the Haagerup property if it has a metrically proper isometric action on some Hilbert space. Examples are $S O(n, 1), S U(n, 1)$ and locally compact, connected, amenable Lie groups. An amenable Lie group is one that has an invariant mean, examples of which include abelian Lie groups, nilpotent Lie groups and solvable Lie groups.

Let $K$ be a maximal compact subgroup of $G$. Let $V$ denote the cotangent space to the symmetric space $G / K$ at the point $(K)$. Let $\operatorname{Cliff}(V)$ be the Clifford algebra of $V$ with respect to some positive definite inner product on $V$. We start by recalling a theorem of Higson-Kasparov [40] and Tu [85], generalizing a theorem of Kasparov [52, $\S 6$ Theorem 2]. 
Theorem 6.1. Let $\mathcal{A}$ be a $G$-C*-algebra, where $G$ is a locally compact, connected Lie group satisfying the Haagerup property. Then in the notation above, the pair of crossed product $C^{*}$-algebras $(\mathcal{A} \rtimes G,(\mathcal{A} \otimes \operatorname{Cliff}(V)) \rtimes K)$ are strongly $\mathrm{KK}$-equivalent. If in addition the coadjoint action of $K$ on $V$ is spin then the pair $\left(\mathcal{A} \rtimes G,(\mathcal{A} \rtimes K) \otimes C_{0}\left(\mathbb{R}^{d}\right)\right)$ are strongly KK-equivalent, where $d=\operatorname{dim}(G / K)$.

A special case of Theorem 6.1, which was proved earlier by Fack and Skandalis [34] generalising an argument of Connes [19], is as follows.

Corollary 6.2. Let $\mathcal{A}$ be a $G$ - $C^{*}$-algebra, where $G$ is a simply connected, locally compact, solvable Lie group of dimension $k$. Then the pair of $C^{*}$-algebras $\left(\mathcal{A} \otimes C_{0}\left(\mathbb{R}^{k}\right)\right.$, $\mathcal{A} \rtimes G)$ are strongly KK-equivalent.

As an immediate consequence of Theorem 6.1 and Lemma 3.4 we obtain the following.

Corollary 6.3. Let $\mathcal{A}$ be a $G$ - $C^{*}$-algebra, where $G$ is a locally compact, connected Lie group satisfying the Haagerup property. Then in the notation of Theorem $6.1,(\mathcal{A} \otimes$ $\mathrm{Cliff}(V)) \rtimes K$ is a (strong, weak) PD algebra if and only if $\mathcal{A} \rtimes G$ is a (strong, weak) $P D$ algebra. If in addition the coadjoint action of $K$ on $V$ is spin, then $\mathcal{A} \rtimes K$ is a (strong, weak) $P D$ algebra if and only if $\mathcal{A} \rtimes G$ is a (strong, weak) PD algebra.

In addition, an immediate consequence of Corollary 6.2 and Lemma 3.4 is as follows.

Corollary 6.4. Let $\mathcal{A}$ be a $G$ - $C^{*}$-algebra, where $G$ is a simply connected, locally compact, solvable Lie group. Then $\mathcal{A}$ is a (strong, weak) PD algebra if and only if $\mathcal{A} \rtimes G$ is a (strong, weak) PD algebra.

Example 6.5. Let $\Gamma$ be a torsion-free, discrete subgroup of a connected semisimple Lie group $G$ with finite center. Let $P$ be a minimal parabolic subgroup of $G$ and $K$ a maximal compact subgroup of $G$. Then $G / P$ is the Furstenberg boundary (at infinity) of the symmetric space $G / K$. By Green's theorem, $C(G / P) \rtimes \Gamma$ is strongly Morita equivalent to $C_{0}(\Gamma \backslash G) \rtimes P$. By Lemmas 3.4 and 3.6 it follows that $C(G / P) \rtimes \Gamma$ is a strong PD algebra if and only if $C_{0}(\Gamma \backslash G) \rtimes P$ is a strong PD algebra. By Corollary 6.3 above, $C_{0}(\Gamma \backslash G) \rtimes P$ is a strong PD algebra if and only if $C_{0}(\Gamma \backslash G) \rtimes K$ is a strong PD algebra, i.e., if and only if $\Gamma \backslash G / K$ is a $\operatorname{spin}^{c}$ manifold. We conclude that $C(G / P) \rtimes \Gamma$ is a strong PD algebra if and only if $\Gamma \backslash G / K$ is a $\operatorname{spin}^{c}$ manifold. In particular, $C\left(\mathbb{S}^{1}\right) \rtimes \Gamma_{g}$ is a strong PD algebra whenever $\Gamma_{g}$ is the fundamental group of a compact, oriented Riemann surface of genus $g \geq 1$. There is a deep variant of this example, analysed in detail by Emerson [32], dealing with the crossed product $C^{*}$-algebra $C(\partial \Gamma) \rtimes \Gamma$ for a hyperbolic group $\Gamma$ with Gromov boundary $\partial \Gamma$.

Another important property of such crossed products is Takai duality. If $G$ is a locally compact, abelian group we denote by $\tilde{G}$ its Pontrjagin dual, i.e., the set of characters of $G$, which is also a locally compact, abelian group. Pontrjagin duality $\tilde{\tilde{G}} \cong G$ follows by Fourier transformation. For example, $\tilde{\mathbb{R}}^{n}=\mathbb{R}^{n}, \tilde{\mathbb{T}}^{n}=\mathbb{Z}^{n}$, and $\tilde{\mathbb{Z}}^{n}=\mathbb{T}^{n}$. If $\mathcal{A}$ is a $G-C^{*}$-algebra, then the crossed product $\mathcal{A} \rtimes G$ carries a $\tilde{G}$-action.

Theorem 6.6 (Takai Duality). Let $\mathcal{A}$ be a $G$ - $C^{*}$-algebra, where $G$ is a locally compact, abelian Lie group. Then there is an isomorphism of $C^{*}$-algebras

$$
(\mathcal{A} \rtimes G) \rtimes \tilde{G} \cong \mathcal{A} \otimes \mathcal{K}\left(L^{2}(G)\right) .
$$


In other words, the algebras $\mathcal{A}$ and $(\mathcal{A} \rtimes G) \rtimes \tilde{G}$ are strongly Morita equivalent. If we interpret the crossed product $\mathcal{A} \rtimes G$ as the noncommutative analogue of an abelian orbifold spacetime $X / G$, then Takai duality asserts that "orbifolding twice" gives back a spacetime which is physically equivalent to the original spacetime $X$. The essential physical phenomenon is that the states which were projected out by $G$ are restored by $\tilde{G}$.

6.2. T-duality and $\mathrm{KK}$-equivalence. We next explain how KK-equivalence of crossed products is related to T-duality. Throughout $X$ will be assumed to be a locally compact, finite-dimensional, homotopically finite space. Consider first the simplest case of flat Dbranes in Type II superstring theory on a spacetime $X=M \times \mathbb{T}^{n}$ which is compactified on an $n$-torus $\mathbb{T}^{n}=\mathbb{V}_{n} / \Lambda_{n}$, where $\Lambda_{n}$ is a lattice of maximal rank in an $n$-dimensional, real vector space $\mathbb{V}_{n}$. As shown in [45], T-duality in this instance is explained by using the correspondence

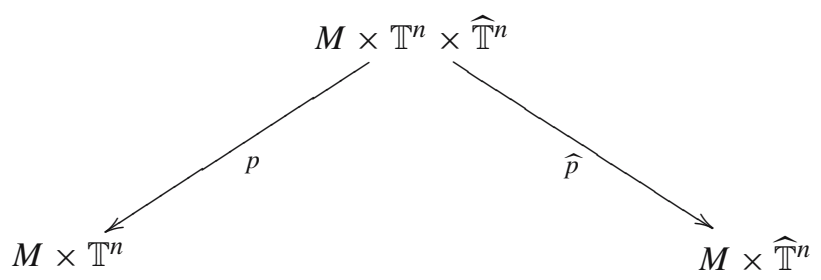

where $\widehat{\mathbb{T}}^{n}=\left(\mathbb{V}_{n}\right)^{\vee} /\left(\Lambda_{n}\right)^{\vee}$ denotes the dual torus, with $\left(\Lambda_{n}\right)^{\vee}$ the dual lattice in the dual vector space $\left(\mathbb{V}_{n}\right)^{\vee}$. This gives rise to an isomorphism of K-theory groups

$$
T !: \mathrm{K}^{\bullet}\left(M \times \mathbb{T}^{n}\right) \stackrel{\approx}{\longrightarrow} \mathrm{K}^{\bullet+n}\left(M \times \widehat{\mathbb{T}}^{n}\right)
$$

given by

$$
T !(-)=\widehat{p} !\left(p^{!}(-) \otimes \mathcal{P}\right),
$$

where $\mathcal{P}$ is the Poincaré line bundle over the torus $\mathbb{T}^{n} \times \widehat{\mathbb{T}}^{n}$ pulled back to $M \times \mathbb{T}^{n} \times \widehat{\mathbb{T}}^{n}$ via the projection map $\mathrm{pr}_{1}: M \times \mathbb{T}^{n} \times \widehat{\mathbb{T}}^{n} \rightarrow \mathbb{T}^{n} \times \widehat{\mathbb{T}}^{n}$. Thus T-duality can be viewed in this case as a smooth analog of the Fourier-Mukai transform. If $G$ is the metric of the torus $\mathbb{T}^{n}$ inherited from the non-degenerate bilinear form of the lattice $\Lambda_{n}$, then the dual torus $\widehat{\mathbb{T}}^{n}$ has metric $G^{-1}$ inherited from the dual lattice $\left(\Lambda_{n}\right)^{\vee}$.

As argued by [63], detailed in [37,44,64,66,86], and discussed in Sect. 1, RR-fields are classified by $\mathrm{K}^{1}$-groups and $\mathrm{RR}$-charges by $\mathrm{K}^{0}$-groups of the spacetime $X$ in Type IIB string theory, whereas RR-fields are classified by $\mathrm{K}^{0}$-groups and RR-charges by $\mathrm{K}^{1}$ groups in Type IIA string theory. Thus if spacetime $X=M \times \mathbb{T}^{n}$ is compactified on a torus of rank $n$, then the isomorphism (6.3) is consistent with the fact that T-duality is an equivalence between the Type IIA and Type IIB string theories if $n$ is odd, while if $n$ is even it is a self-duality for both string theories. Given this compelling fact, we will take this isomorphism to mean the equivalence itself here (although in string theory the duality is much more complicated and involves many more ingredients).

As was observed in [59], all of this can be reformulated in terms of the $C^{*}$-algebra $C_{0}\left(M \times \mathbb{T}^{n}\right)$. The locally compact, abelian vector Lie group $\mathbb{V}_{n} \cong \mathbb{R}^{n}$ acts on $\mathbb{T}^{n}=$ $\mathbb{V}_{n} / \Lambda_{n}$ via left translations, and consider the crossed product algebra $C_{0}\left(M \times \mathbb{T}^{n}\right) \rtimes \mathbb{V}_{n}$ with $\mathbb{V}_{n}$ acting trivially on $M$. By Rieffel's imprimitivity theorem [76], there is a strong Morita equivalence

$$
C_{0}\left(M \times \mathbb{T}^{n}\right) \rtimes \mathbb{V}_{n} \sim C_{0}(M) \rtimes \Lambda_{n},
$$


where the discrete group $\Lambda_{n}$ acts trivially on $C_{0}(M)$. One therefore has

$$
C_{0}\left(M \times \mathbb{T}^{n}\right) \rtimes \mathbb{V}_{n} \sim C_{0}(M) \otimes C^{*}\left(\Lambda_{n}\right) .
$$

By Fourier transformation the group $C^{*}$-algebra of $\Lambda_{n}$ can be identified as $C^{*}\left(\Lambda_{n}\right) \cong$ $C\left(\widehat{\mathbb{T}}^{n}\right)$, and as a consequence there is a strong Morita equivalence

$$
C_{0}\left(M \times \mathbb{T}^{n}\right) \rtimes \mathbb{V}_{n} \sim C_{0}(M) \otimes C\left(\widehat{\mathbb{T}}^{n}\right) \cong C_{0}\left(M \times \widehat{\mathbb{T}}^{n}\right) .
$$

By Lemma 3.6 and Corollary 6.2, the pair of $C^{*}$-algebras $\left(C_{0}\left(M \times \mathbb{T}^{n}\right), C_{0}(M \times\right.$ $\left.\left.\mathbb{T}^{n}\right) \rtimes \mathbb{V}_{n} \sim C_{0}\left(M \times \widehat{\mathbb{T}}^{n}\right)\right)$ are strongly KK-equivalent (with a degree shift of $\left.n \bmod 2\right)$. Thus by Lemma 3.2 there are isomorphisms

$$
\begin{aligned}
& T_{!}: \mathrm{K}_{\bullet}\left(C_{0}\left(M \times \mathbb{T}^{n}\right)\right) \stackrel{\approx}{\longrightarrow} \mathrm{K}_{\bullet+n}\left(C_{0}\left(M \times \widehat{\mathbb{T}}^{n}\right)\right), \\
& T^{!}: \mathrm{K}^{\bullet}\left(C_{0}\left(M \times \mathbb{T}^{n}\right)\right) \stackrel{\approx}{\longrightarrow} \mathrm{K}^{\bullet+n}\left(C_{0}\left(M \times \widehat{\mathbb{T}}^{n}\right)\right) .
\end{aligned}
$$

The upshot of this analysis is that the Fourier-Mukai transform, or equivalently T-duality for flat D-branes in Type II string theory, on a spacetime $X$ that is compactified on a torus $\mathbb{T}^{n}$, can be interpreted as taking a crossed product with the natural action of $\mathbb{V}_{n} \cong \mathbb{R}^{n}$ on the $C^{*}$-algebra $C_{0}(X)$.

This point of view was generalized in a series of papers [9-12,59,60] to twisted D-branes in Type II superstring theory in a $B$-field $(X, H)$ and for a spacetime $X$ which is a possibly non-trivial principal torus bundle $\pi: X \rightarrow M$ of rank $n$. As described in Sect. 1.4, in this case the type I, separable $C^{*}$-algebra in question is the algebra $C_{0}\left(X, \mathcal{E}_{H}\right)$ of continuous sections vanishing at infinity of a locally trivial $C^{*}$-algebra bundle $\mathcal{E}_{H} \rightarrow X$ with fibre $\mathcal{K}(\mathcal{H})$ and Dixmier-Douady invariant $\delta_{X}\left(\mathcal{E}_{H}\right)=H \in$ $\mathrm{Br}^{\infty}(X) \cong \mathrm{H}^{3}(X, \mathbb{Z})$. This is a stable, continuous trace algebra with spectrum $X$. It is a fundamental theorem of Dixmier and Douady [31] that $H$ is trivial in cohomology if and only if $C_{0}\left(X, \mathcal{E}_{H}\right)$ is strongly Morita equivalent to $C_{0}(X)$ (in fact $C_{0}\left(X, \mathcal{E}_{0}\right) \cong$ $\left.C_{0}(X, \mathcal{K}(\mathcal{H}))\right)$, consistent with the above discussion. If in addition $X$ is a Calabi-Yau threefold, then T-duality in these instances coincides with mirror symmetry.

As above, the abelian Lie group $\mathbb{V}_{n}$ acts on $X$ via left translations of the fibres $\mathbb{T}^{n}$. In [60] the following fundamental technical theorem was proven.

Theorem 6.7. In the notation above, the natural $\mathbb{V}_{n}$-action on $X$ lifts to a $\mathbb{V}_{n}$-action on the total space $\mathcal{E}_{H}$, and hence to a $\mathbb{V}_{n}$-action on $C_{0}\left(X, \mathcal{E}_{H}\right)$, if and only if the restriction of $H$ to the fibres of $X$ is trivial in cohomology.

This is a non-trivial obstruction if and only if the fibres of $X$ are of rank $n \geq 3$. The T-dual is then defined as the crossed product algebra $C_{0}\left(X, \mathcal{E}_{H}\right) \rtimes \mathbb{V}_{n}$. This algebra is a continuous trace algebra if and only if $\pi_{*}(H)=0$ in $\mathrm{H}^{1}\left(M, \mathrm{H}^{2}\left(\mathbb{T}^{n}, \mathbb{Z}\right)\right)$. In the general case, the crossed product $C_{0}\left(X, \mathcal{E}_{H}\right) \rtimes \mathbb{V}_{n}$ is not of type I but is rather a continuous field of (stabilized) rank $n$ noncommutative tori fibred over $M$. The fibre over the point $m \in M$ is isomorphic to $\mathcal{T}_{f(m)}^{n} \otimes \mathcal{K}(\mathcal{H})$, where $\pi_{*}(H)=[f]$ is the Mackey obstruction class with $f: M \rightarrow \mathrm{H}^{2}\left(\Lambda_{n}, U(1)\right) \cong(\mathbb{R} / \mathbb{Z})^{k}, k=\left(\begin{array}{l}n \\ 2\end{array}\right)$ a continuous map. This obstruction is due to the presence of discrete torsion in the fibres of the string background, represented by multipliers $f(m)$ on the discrete group $\Lambda_{n}$, which is essentially due to the presence of non-trivial global $B$-fields along the fibres of $X$.

Corollary 6.8. In the notation of Theorem 6.7, suppose that the restriction of $H$ to the fibres of $X$ is trivial in cohomology. Then the T-dual $C_{0}\left(X, \mathcal{E}_{H}\right) \rtimes \mathbb{V}_{n}$ is a strong $P D$ algebra if and only if $X$ is a spin manifold. $^{c}$ 
Proof. By a theorem of Parker [67], the continuous trace algebra $C_{0}\left(X, \mathcal{E}_{H}\right)$ is a strong $\mathrm{PD}$ algebra if and only if $X$ is a $\operatorname{spin}^{c}$ manifold. The result now follows from Theorem 6.7 and Corollary 6.4.

The Takai duality theorem in these examples implies that $\left(C_{0}\left(X, \mathcal{E}_{H}\right) \rtimes \mathbb{V}_{n}\right) \rtimes \mathbb{V}_{n}$ is strongly Morita equivalent to $C_{0}\left(X, \mathcal{E}_{H}\right)$, i.e., T-duality applied twice returns the original string theory. We can now combine all of these observations to formulate a generically noncommutative version of T-duality for $C^{*}$-algebras in very general settings.

Definition 6.9 (K-Theoretic T-Duality). Let $\underline{\mathfrak{T}}$ be a suitable category of separable $C^{*}$ algebras, possibly equipped with some extra structure (such as the $\mathbb{R}^{n}$-action above). Elements of $\mathfrak{T}$ are called $\mathbf{T}$-dualizable algebras, with the following properties:

(1) There is a covariant functor $\mathrm{T}: \underline{T} \rightarrow \underline{\mathfrak{T}}$ which sends an algebra $\mathcal{A}$ to an algebra $\mathrm{T}(\mathcal{A})$ called its $\mathbf{T}$-dual;

(2) There is a functorial map $\mathcal{A} \mapsto \gamma_{\mathcal{A}} \in \mathrm{KK}_{n}(\mathcal{A}, \mathrm{T}(\mathcal{A}))$ such that $\gamma_{\mathcal{A}}$ is a $\mathrm{KK}$-equivalence; and

(3) The pair $\left(\mathcal{A}, \mathrm{T}(\mathrm{T}(\mathcal{A}))\right.$ ) are Morita equivalent, and the Kasparov product $\gamma_{\mathcal{A}} \otimes_{\mathrm{T}}(\mathcal{A})$ $\gamma_{\mathrm{T}(\mathcal{A})}$ is the $\mathrm{KK}$-equivalence associated to this Morita equivalence.

6.3. T-duality and $\mathrm{HL}$-equivalence. As we have mentioned, the isomorphisms (6.8) are only part of the story behind T-duality, as they only dictate how topological charges behave under the duality. In particular, the isomorphism $T$ ! on K-theory bijectively relates the RR-fields in T-dual spacetimes, while the bijection $T^{!}$relates the RR-charges themselves. As explained in Sects. 1.1 and 1.2 in the case of flat D-branes, the RR-fields are represented by closed differential forms on the spacetime $X$ while the branes themselves are associated to non-trivial (worldvolume) cycles of $X$. It is therefore natural to attempt to realize our characterization of T-duality above in the language of cyclic theory, in order to provide the bijections between the analogues of these (and other) geometric structures.

We begin with the following observation.

Theorem 6.10. Let $\mathcal{A}$ be separable $C^{*}$-algebra, and suppose that $\mathcal{A}$ admits an action by a locally compact, real, abelian vector Lie group $\mathbb{V}_{n}$ of dimension $n$. Then there is a commutative diagram

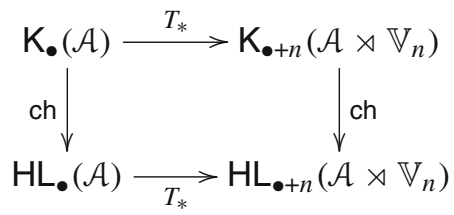

whose horizontal arrows are isomorphisms.

Proof. The isomorphism $\mathrm{K}_{\bullet}(\mathcal{A}) \cong \mathrm{K}_{\bullet+n}\left(\mathcal{A} \rtimes \mathbb{R}^{n}\right)$ in the top row is the Connes-Thom isomorphism [19] (cf. Corollary 6.2 above), while the isomorphism in the bottom row comes from transporting this isomorphism to local cyclic homology, as in [82]. (See also the review of [82] in MathSciNet, MR2117221 (2005j:46041), for more of an explanation.) 
The isomorphism in the bottom row of (6.9) is the local cyclic homology version of T-duality. Theorem 6.10 shows that the T-duality isomorphisms in K-theory descend to isomorphisms of cyclic cohomology, giving the mappings at the level of RR-field representatives in $\mathrm{HL}_{\bullet}(\mathcal{A})$. This motivates the local cyclic cohomology version of the axioms spelled out in Definition 6.9.

Definition 6.11 (Cohomological T-Duality). Let $\mathcal{A}$ be a complete bornological algebra. A cyclic T-dual of $\mathcal{A}$ is a complete bornological algebra $\mathrm{T}^{\mathrm{HL}}(\mathcal{A})$ which satisfies the following three axioms:

(1) The map $\mathcal{A} \mapsto \mathrm{T}^{\mathrm{HL}}(\mathcal{A})$ is a covariant functor on an appropriate category of algebras;

(2) The pair $\left(\mathcal{A}, \mathrm{T}^{\mathrm{HL}}(\mathcal{A})\right)$ are $\mathrm{HL}$-equivalent; and

(3) The pair $\left(\mathcal{A}, \mathrm{T}^{\mathrm{HL}}\left(\mathrm{T}^{\mathrm{HL}}(\mathcal{A})\right)\right.$ ) are topologically Morita equivalent.

As in Definition 6.9, there should be an explicit functorial HL-equivalence in (2) compatible with the Morita equivalence in (3).

From these definitions it follows that K-theoretic T-duality for a separable $C^{*}$-algebra $\mathcal{A}$ implies cohomological T-duality for the same algebra, but the converse need not necessarily be true (because of torsion in K-theory, for example).

Remark 6.12. There are competing points of view concerning T-duality in the nonclassical case, that is, in the case when the T-dual of a spacetime $X$, which is a principal torus bundle with nontrivial $H$-flux, is not another principal torus bundle. Unlike the approach discussed in this section, where the T-dual is a globally defined but possibly noncommutative algebra, the T-dual in the competing points of view is not globally defined. For example, in [46], Hitchin's generalized complex geometry is used to construct a T-dual which is a purely local object, that does not patch together to give a global object, and is referred to as a T-fold. See also [81] for a related point of view.

\section{Todd Classes and Gysin Maps}

In this section we apply the concept of Poincaré duality in KK-theory and bivariant cyclic cohomology to define the notion of a Todd class for a very general class of $C^{*}$-algebras. As follows from the discussion of Sect. 1.1, this will be one of the main building blocks of our definitions of generalized D-brane charges. Another crucial ingredient in these definitions is the application of Poincare duality to the construction of Gysin maps (or "wrong way" maps) in KK-theory and in cyclic theory, which also came up in our discussion of T-duality above. These general constructions combine together to yield a generalization of the Grothendieck-Riemann-Roch theorem for an appropriate class of $C^{*}$-algebras, which in turn yields another perspective on the concept of a T-dual $C^{*}$-algebra that was introduced in the previous section.

7.1. The Todd class. Our general definition of Todd classes is motivated by Theorem 5.5 and Proposition 2.8. We begin with the following observation.

Lemma 7.1. Let $\mathcal{A}, \mathcal{B}_{1}, \mathcal{B}_{2}$ be separable $C^{*}$-algebras such that $\left(\mathcal{A}, \mathcal{B}_{1}\right)$ and $\left(\mathcal{A}, \mathcal{B}_{2}\right)$ are both strong PD pairs. Then the pair of algebras $\left(\mathcal{B}_{1}, \mathcal{B}_{2}\right)$ are strongly $\mathrm{KK}$-equivalent. 
Proof. Let $\Delta_{1} \in \mathrm{K}^{d}\left(\mathcal{A} \otimes \mathcal{B}_{1}\right)$ and $\Delta_{2} \in \mathrm{K}^{d}\left(\mathcal{A} \otimes \mathcal{B}_{2}\right)$ be the respective fundamental classes. Then with a proof along the lines of Proposition 2.8, one shows that the classes $\alpha:=\Delta_{1}^{\vee} \otimes_{\mathcal{A}} \Delta_{2} \in \mathrm{KK}_{0}\left(\mathcal{B}_{1}, \mathcal{B}_{2}\right)$ and $\beta:=(-1)^{d} \Delta_{2}^{\vee} \otimes_{\mathcal{A}} \Delta_{1} \in \mathrm{KK}_{0}\left(\mathcal{B}_{2}, \mathcal{B}_{1}\right)$ implement the required equivalence.

Let $\mathfrak{D}$ denote the class of all separable $C^{*}$-algebras $\mathcal{A}$ for which there exists another separable $C^{*}$-algebra $\mathcal{B}$ such that $(\mathcal{A}, \mathcal{B})$ is a strong PD pair. For any such $\mathcal{A}$, we fix a representative of the $\mathrm{KK}$-equivalence class of $\mathcal{B}$ and denote it by $\tilde{\mathcal{A}}$. In general there is no canonical choice for $\tilde{\mathcal{A}}$. If $\mathcal{A}$ is a strong PD algebra, the canonical choice $\tilde{\mathcal{A}}:=\mathcal{A}^{\mathrm{o}}$ will always be made.

Definition 7.2. Let $\mathcal{A} \in \underline{\mathfrak{D}}$, let $\Delta \in \mathrm{K}^{d}(\mathcal{A} \otimes \tilde{\mathcal{A}})$ be a fundamental $\mathrm{K}$-homology class for the pair $(\mathcal{A}, \tilde{\mathcal{A}})$ and let $\Xi \in \mathrm{HL}^{d}(\mathcal{A} \otimes \tilde{\mathcal{A}})$ be a fundamental cyclic cohomology class. Then the Todd class of $\mathcal{A}$ is defined to be the class

$$
\operatorname{Todd}(\mathcal{A})=\operatorname{Todd}_{\Delta, \Xi}(\mathcal{A}, \tilde{\mathcal{A}}):=\Xi^{\vee} \otimes_{\tilde{\mathcal{A}}} \operatorname{ch}(\Delta)
$$

in the ring $\mathrm{HL}_{0}(\mathcal{A}, \mathcal{A})$.

Recall that the map $\Xi^{\vee} \otimes \tilde{\mathcal{A}}(-)$ implements an isomorphism $\mathrm{HL}^{d}(\mathcal{A} \otimes \tilde{\mathcal{A}}) \cong \mathrm{HL}_{0}(\mathcal{A}, \mathcal{A})$. The element (7.1) is invertible with inverse given by

$$
\operatorname{Todd}(\mathcal{A})^{-1}=(-1)^{d} \operatorname{ch}\left(\Delta^{\vee}\right) \otimes_{\tilde{\mathcal{A}}} \Xi .
$$

Remark 7.3. Observe that the Todd class of an algebra $\mathcal{A} \in \mathfrak{D}$ is trivial, i.e., $\operatorname{Todd}(\mathcal{A})=$ $1_{\mathcal{A}}$ in $\mathrm{HL}_{0}(\mathcal{A}, \mathcal{A})$, if and only if $\operatorname{ch}(\Delta)=\Xi$ in $\mathrm{HL}^{d}(\mathcal{A} \otimes \tilde{\mathcal{A}})$.

The Todd class depends on a number of choices, but this dependence can be described by "covariant" actions on the classes.

Theorem 7.4. In the notation above, the Todd class of an algebra $\mathcal{A} \in \mathfrak{D}$ has the following properties:

(1) Suppose $\Delta$ and $\Xi$ are fundamental classes for the strong $\operatorname{PD}$ pair $(\mathcal{A}, \tilde{\mathcal{A}})$, with inverse fundamental classes $\Delta^{\vee}$ and $\Xi^{\vee}$. If there are $\mathrm{KK}$-equivalences $\alpha \in \mathrm{KK}_{0}(\mathcal{A}$, $\left.\mathcal{A}_{1}\right)$ and $\beta \in \mathrm{KK}_{0}\left(\tilde{\mathcal{A}}, \tilde{\mathcal{A}}_{1}\right)$, then $\left(\mathcal{A}_{1}, \tilde{\mathcal{A}}_{1}\right)$ is a strong PD pair, with fundamental classes

$$
\Delta_{1}=\left(\alpha^{-1} \times \beta^{-1}\right) \otimes_{\mathcal{A} \otimes \tilde{\mathcal{A}}} \Delta, \quad \Xi_{1}=\left(\operatorname{ch}(\alpha)^{-1} \times \operatorname{ch}(\beta)^{-1}\right) \otimes_{\mathcal{A} \otimes \tilde{\mathcal{A}}} \Xi
$$

(where $\times$ denotes the exterior product) and inverse fundamental classes

$$
\Delta_{1}^{\vee}=\Delta^{\vee} \otimes_{\mathcal{A} \otimes \tilde{\mathcal{A}}}(\alpha \times \beta), \quad \Xi_{1}^{\vee}=\Xi^{\vee} \otimes_{\mathcal{A} \otimes \tilde{\mathcal{A}}}(\operatorname{ch}(\alpha) \times \operatorname{ch}(\beta)) .
$$

Furthermore,

$$
\operatorname{Todd}_{\Delta_{1}, \Xi_{1}}\left(\mathcal{A}_{1}, \tilde{\mathcal{A}}_{1}\right)=\operatorname{ch}(\alpha)^{-1} \otimes_{\mathcal{A}} \operatorname{Todd}_{\Delta, \Xi}(\mathcal{A}, \tilde{\mathcal{A}}) \otimes_{\mathcal{A}} \operatorname{ch}(\alpha) .
$$

(2) If $\left(\ell, \ell_{\mathrm{HL}}\right)$ is an element of the duality group $\mathrm{KK}_{0}(\mathcal{A}, \mathcal{A})^{-1} \times \mathrm{HL}_{0}(\mathcal{A}, \mathcal{A})^{-1}$, then

$$
\operatorname{Todd}_{\ell \otimes_{\mathcal{A}} \Delta, \ell_{\mathrm{HL}} \otimes_{\mathcal{A}} \Xi}(\mathcal{A}, \tilde{\mathcal{A}})=\operatorname{ch}(\ell) \otimes_{\mathcal{A}} \operatorname{Todd}_{\Delta, \Xi}(\mathcal{A}, \tilde{\mathcal{A}}) \otimes_{\mathcal{A}} \ell_{\mathrm{HL}}^{-1} .
$$


Proof. The fact that $\left(\mathcal{A}_{1}, \tilde{\mathcal{A}}_{1}\right)$ is a strong PD pair with fundamental classes $\Delta_{1}$ and $\Xi_{1}$ is routine and quite similar to the calculation in Proposition 2.7. We proceed to compute the Todd class. In terms of the diagram calculus of Appendix B, the picture is:
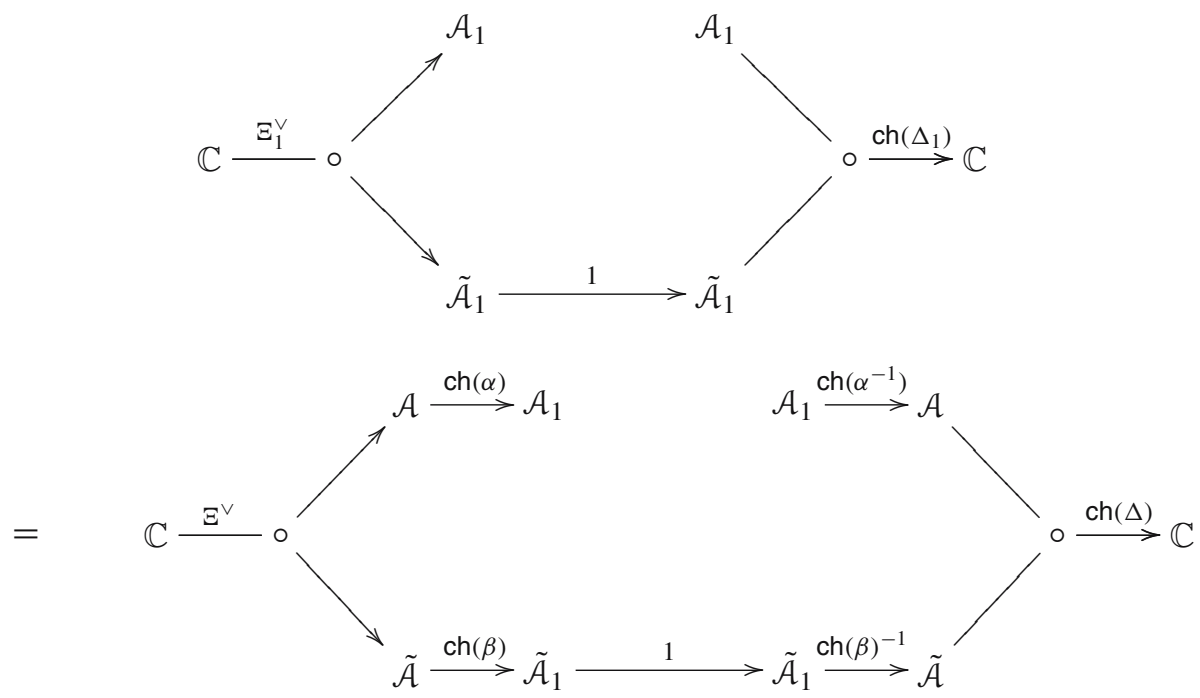

This yields formula (7.3) via associativity in the formulation of Appendix B.

The proof of (2) is done with a very similar diagram.

Corollary 7.5. Suppose $\mathcal{A}$ is a $C^{*}$-algebra that is strongly $\mathrm{KK}$-equivalent to $C(X)$, where $X$ is an even-dimensional compact $\operatorname{spin}^{c}$ manifold. Let Todd $(X)$ be the usual Todd class of $X$, but viewed as a bivariant cyclic homology class as above. If $\alpha \in \mathrm{KK}(\mathcal{A}, C(X))$ and $\beta \in \mathrm{KK}(C(X), \mathcal{A})$ are explicit $\mathrm{KK}$-equivalences inverse to one another, then

$$
\operatorname{Todd}(\mathcal{A})=\operatorname{ch}(\alpha) \otimes_{C(X)} \operatorname{Todd}(X) \otimes_{C(X)} \operatorname{ch}(\beta) .
$$

Proof. Immediate from Theorem 7.4, since $D_{X \times X}$ is a KK fundamental class for $C(X)$ and the usual homology fundamental class provides another fundamental class in cyclic homology.

Remark 7.6. There is a more subtle real version of Kasparov's KK-theory defined for complex $C^{*}$-algebras with involution [39, Definition 9.18]. For any separable $C^{*}$-algebra $\mathcal{A}$, the algebra $\mathcal{A} \otimes \mathcal{A}^{\mathrm{o}}$ may be equipped with a canonical involution $\tau$ defined by

$$
\tau\left(a \otimes b^{\mathrm{o}}\right)=b^{*} \otimes\left(a^{*}\right)^{\mathrm{o}}
$$

for all $a, b \in \mathcal{A}$. The corresponding real K-homology groups are denoted $\mathrm{KR}^{\bullet}\left(\mathcal{A} \otimes \mathcal{A}^{\mathrm{o}}\right)$. There is a forgetful map

$$
\mathfrak{f}: \mathrm{KR}^{\bullet}\left(\mathcal{A} \otimes \mathcal{A}^{\mathrm{o}}\right) \longrightarrow \mathrm{K}^{\bullet}\left(\mathcal{A} \otimes \mathcal{A}^{\mathrm{o}}\right)
$$

from the real to the complex K-homology of the algebra $\mathcal{A} \otimes \mathcal{A}^{\circ}$. Suppose that $\mathcal{A}$ is a strong PD algebra which admits a fundamental KR-homology class $\Delta_{\mathbb{R}} \in \mathrm{KR}^{d}\left(\mathcal{A} \otimes \mathcal{A}^{\mathrm{o}}\right)$. Let $\Xi \in \mathrm{HL}^{d}\left(\mathcal{A} \otimes \mathcal{A}^{\mathrm{o}}\right)$ be a fundamental cyclic cohomology class for $\mathcal{A}$. The image of 
$\Delta_{\mathbb{R}}$ under the homomorphism (7.8) is a fundamental K-homology class for $\mathcal{A}$ and the corresponding Todd class $(7.1)$ in $\operatorname{HL}_{0}(\mathcal{A}, \mathcal{A})$ is called the Atiyah-Hirzebruch class of $\mathcal{A}$, denoted

$$
\widehat{A}(\mathcal{A}):=\Xi^{\vee} \otimes_{\mathcal{A}^{\mathrm{o}}} \operatorname{ch}\left(\mathfrak{f}\left(\Delta_{\mathbb{R}}\right)\right) .
$$

It satisfies the same basic properties as the Todd class above, and may be related to (7.1) for any other fundamental $\mathrm{K}$-homology class $\Delta$ by using the action of the duality group $\mathrm{KK}_{0}(\mathcal{A}, \mathcal{A})^{-1}$ in $(7.4)$.

7.2. Gysin homomorphisms. Let $f: \mathcal{A} \rightarrow \mathcal{B}$ be a morphism of separable $C^{*}$-algebras. It induces morphisms in K-theory,

$$
f_{*}: \mathrm{K}_{\bullet}(\mathcal{A}) \longrightarrow \mathrm{K}_{\bullet}(\mathcal{B})
$$

and morphisms in K-homology,

$$
f^{*}: \mathrm{K}^{\bullet}(\mathcal{B}) \longrightarrow \mathrm{K}^{\bullet}(\mathcal{A})
$$

We will now describe how to construct Gysin maps (or "wrong way" maps) on these groups. If both $\mathcal{A}$ and $\mathcal{B}$ are PD algebras, then they are easily constructed as analogues of the classical "Umkehrhomomorphismus". In this case, there are isomorphisms

$$
\operatorname{Pd}_{\mathcal{A}}: \mathrm{K}_{\bullet}(\mathcal{A}) \stackrel{\approx}{\longrightarrow} \mathrm{K}^{\bullet-d_{\mathcal{A}}}\left(\mathcal{A}^{\mathrm{o}}\right) \text { and } \operatorname{Pd}_{\mathcal{B}}: \mathrm{K}_{\bullet}(\mathcal{B}) \stackrel{\approx}{\longrightarrow} \mathrm{K}^{\bullet-d_{\mathcal{B}}}\left(\mathcal{B}^{\mathrm{o}}\right) .
$$

We can then define the Gysin map in K-theory,

$$
f !: \mathrm{K}_{\bullet}(\mathcal{B}) \longrightarrow \mathrm{K}_{\bullet+d}(\mathcal{A}),
$$

(where $d=d_{\mathcal{A}}-d_{\mathcal{B}}$ ) as the composition

$$
f_{!}: \mathrm{K}_{\bullet}(\mathcal{B}) \stackrel{\mathrm{Pd}_{\mathcal{B}}}{\longrightarrow} \mathrm{K}^{\bullet-d_{\mathcal{B}}}\left(\mathcal{B}^{\mathrm{o}}\right) \stackrel{\left(f^{\circ}\right)^{*}}{\longrightarrow} \mathrm{K}^{\bullet-d_{\mathcal{B}}}\left(\mathcal{A}^{\mathrm{o}}\right) \stackrel{\mathrm{Pd}_{\mathcal{A}}^{-1}}{\longrightarrow} \mathrm{K}_{\bullet+d}(\mathcal{A}) .
$$

Under the same hypotheses, we can similarly define the Gysin map in K-homology,

$$
f^{!}: \mathrm{K}^{\bullet}(\mathcal{A}) \longrightarrow \mathrm{K}^{\bullet+d}(\mathcal{B})
$$

as the composition

$$
f^{!}: \mathrm{K}^{\bullet}(\mathcal{A}) \stackrel{\mathrm{Pd}_{\mathcal{A}}^{-1}}{\longrightarrow} \mathrm{K}_{\bullet+d_{\mathcal{A}}}\left(\mathcal{A}^{\mathrm{o}}\right) \stackrel{f_{*}^{\mathrm{o}}}{\longrightarrow} \mathrm{K}_{\bullet}+d_{\mathcal{A}}\left(\mathcal{B}^{\mathrm{o}}\right) \stackrel{\mathrm{Pd}_{\mathcal{B}}}{\longrightarrow} \mathrm{K}^{\bullet+d}(\mathcal{B})
$$

However, this relies on the fact that $\mathcal{A}$ and $\mathcal{B}$ are PD algebras, which is in general too stringent a requirement. We will therefore proceed to some more general constructions. 


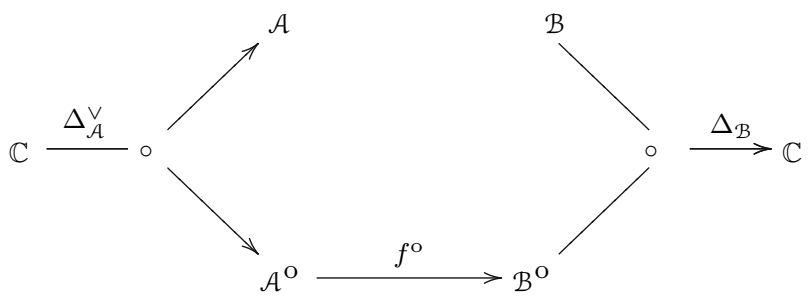

Fig. 7.2. Diagram representing the construction of $f$ !. The "free ends" are on the top line and concatenation is done on the bottom line.

7.3. Strongly K-oriented maps. We will consider a subcategory $\mathfrak{C}$ of the category of separable $C^{*}$-algebras and morphisms of $C^{*}$-algebras, consisting of strongly $\mathrm{K}$-oriented morphisms. $\underline{\mathfrak{C}}$ comes equipped with a contravariant functor $!: \underline{\mathfrak{C}} \rightarrow \underline{\mathrm{KK}}$, sending

$$
\underline{\mathfrak{C}} \ni(\mathcal{A} \stackrel{f}{\longrightarrow} \mathcal{B}) \longrightarrow f ! \in \mathrm{KK}_{d}(\mathcal{B}, \mathcal{A})
$$

and having the following properties:

(1) For any $C^{*}$-algebra $\mathcal{A}$, the identity morphism $\operatorname{id}_{\mathcal{A}}: \mathcal{A} \rightarrow \mathcal{A}$ is strongly K-oriented with $\left(\mathrm{id}_{\mathcal{A}}\right) !=1_{\mathcal{A}}$, and the 0 -morphism $0_{\mathcal{A}}: \mathcal{A} \rightarrow 0$ is strongly K-oriented with $\left(0_{\mathcal{A}}\right) !=0 \in \operatorname{KK}(0, \mathcal{A})$;

(2) If $(\mathcal{A} \stackrel{f}{\longrightarrow} \mathcal{B}) \in \underline{\mathfrak{C}}$, then $\left(\mathcal{A}^{\mathrm{o}} \stackrel{f^{\mathrm{o}}}{\longrightarrow} \mathcal{B}^{\mathrm{o}}\right) \in \underline{\mathfrak{C}}$ and moreover $(f !)^{\mathrm{o}}=\left(f^{\mathrm{o}}\right)$ !;

(3) If $\mathcal{A}$ and $\mathcal{B}$ are strong PD algebras, then any morphism $(\mathcal{A} \stackrel{f}{\longrightarrow} \mathcal{B}) \in \underline{C}$, and $f$ ! is determined as follows:

$$
f !=(-1)^{d_{\mathcal{A}}} \Delta_{\mathcal{A}}^{\vee} \otimes_{\mathcal{A}^{0}}\left[f^{\mathrm{o}}\right] \otimes_{\mathcal{B}^{\circ}} \Delta_{\mathcal{B}},
$$

where for the rest of the paper, $[f]=\mathrm{KK}(f)$ denotes the class in $\mathrm{KK}(\mathcal{A}, \mathcal{B})$ of the $\operatorname{morphism}(\mathcal{A} \stackrel{f}{\longrightarrow} \mathcal{B})$ and $\left[f^{\circ}\right]$ is defined similarly.

As Kasparov products like this are rather hard to visualize when written this way, it is useful to use the diagram calculus developed in Appendix B. In these terms, $f$ ! is represented by the picture depicted by Fig. 7.2.

Actually, it is not immediately obvious that property (3) above is compatible with the required functoriality. However, consistency of the definition follows from the following:

Lemma 7.7 (Functoriality of the Gysin map). If $\mathcal{A}, \mathcal{B}$ and $\mathcal{C}$ are strong PD algebras, and if $f: \mathcal{A} \rightarrow \mathcal{B}, g: \mathcal{B} \rightarrow \mathcal{C}$ are morphisms of $C^{*}$-algebras, then

$$
\begin{array}{r}
\left((-1)^{d_{\mathcal{A}}} \Delta_{\mathcal{A}}^{\vee} \otimes_{\mathcal{A}^{\circ}}\left[f^{\mathrm{o}}\right] \otimes_{\mathcal{B}^{\circ}} \Delta_{\mathcal{B}}\right) \otimes_{\mathcal{B}}\left((-1)^{d_{\mathcal{B}}} \Delta_{\mathcal{B}}^{\vee} \otimes_{\mathcal{B}^{\circ}}\left[g^{\mathrm{o}}\right] \otimes_{\mathcal{C}^{\circ}} \Delta_{\mathcal{C}}\right) \\
=\left((-1)^{d_{\mathcal{A}}} \Delta_{\mathcal{A}}^{\vee} \otimes_{\mathcal{A}^{\circ}}\left[(g \circ f)^{\mathrm{o}}\right] \otimes_{\mathcal{C}^{\circ}} \Delta_{\mathcal{C}}\right) .
\end{array}
$$

Proof. Note that, by associativity of the Kasparov product,

$$
\begin{aligned}
& \left(\Delta_{\mathcal{A}}^{\vee} \otimes_{\mathcal{A}^{\circ}}\left[f^{\mathrm{o}}\right] \otimes_{\mathcal{B}^{\circ}} \Delta_{\mathcal{B}}\right) \otimes_{\mathcal{B}}\left(\Delta_{\mathcal{B}}^{\vee} \otimes_{\mathcal{B}^{\circ}}\left[g^{\mathrm{o}}\right] \otimes_{\mathcal{C}^{\mathrm{o}}} \Delta_{\mathcal{C}}\right) \\
= & \Delta_{\mathcal{A}^{\vee}}^{\vee} \otimes_{\mathcal{A}^{\mathrm{o}}}\left(\left[f^{\mathrm{o}}\right] \otimes_{\mathcal{B}^{\circ}} \Delta_{\mathcal{B}} \otimes_{\mathcal{B}} \Delta_{\mathcal{B}}^{\vee} \otimes_{\mathcal{B}^{\circ}}\left[g^{\mathrm{o}}\right]\right) \otimes_{\mathcal{C}^{\circ}} \Delta_{\mathcal{C}} .
\end{aligned}
$$


But

$$
\begin{aligned}
{\left[f^{\mathrm{o}}\right] \otimes_{\mathcal{B}^{\circ}} \Delta_{\mathcal{B}} \otimes_{\mathcal{B}} \Delta_{\mathcal{B}}^{\vee} \otimes_{\mathcal{B}^{0}}\left[g^{\mathrm{o}}\right] } & =\left[f^{\mathrm{o}}\right] \otimes_{\mathcal{B}^{0}}\left(\Delta_{\mathcal{B}} \otimes_{\mathcal{B}} \Delta_{\mathcal{B}}^{\vee}\right) \otimes_{\mathcal{B}^{0}}\left[g^{\mathrm{o}}\right] \\
& =\left[f^{\mathrm{o}}\right] \otimes_{\mathcal{B}^{0}}(-1)^{d_{\mathcal{B}}} 1_{\mathcal{B}^{\circ}} \otimes_{\mathcal{B}^{\circ}}\left[g^{\mathrm{o}}\right] \\
& =(-1)^{d_{\mathcal{B}}}\left[f^{\mathrm{o}}\right] \otimes_{\mathcal{B}^{\circ}}\left[g^{\mathrm{o}}\right]=(-1)^{d_{\mathcal{B}}}\left[(g \circ f)^{\mathrm{o}}\right],
\end{aligned}
$$

and the result follows.

We now exhibit more examples of elements in this category $\mathfrak{C}$. In the following example, the $C^{*}$-algebras are not strong PD algebras, but yet we can get an element in this category $\mathfrak{C}$. Suppose that we are given oriented manifolds $X$ and $Y$, and classes $H_{X} \in \mathrm{H}^{3}(X, \mathbb{Z})$ and $H_{Y} \in \mathrm{H}^{3}(Y, \mathbb{Z})$. A smooth map $f: X \rightarrow Y$ defines a morphism $f^{*}: C_{0}\left(Y, \mathcal{E}_{H_{Y}}\right) \longrightarrow C_{0}\left(X, \mathcal{E}_{H_{X}}\right)$ if $f^{*} H_{Y}=H_{X}$. Since $X$ and $Y$ are oriented, then by Example 2.5, the pair $\left(C_{0}(X), C_{0}(X, \operatorname{Cliff}(T X))\right)$ is a strong PD pair, that is, there is a fundamental class $\Delta_{X} \in \mathrm{KK}\left(C_{0}(X) \otimes C_{0}(X, \operatorname{Cliff}(T X), \mathbb{C})\right.$. Since $\mathcal{E}_{H_{X}} \otimes \mathcal{E}_{H_{X}}^{\mathrm{o}}$ is stably isomorphic to the trivial bundle $X \times \mathcal{K}$, it follows that $C_{0}(X) \otimes C_{0}(X, \operatorname{Cliff}(T X)$ is stably isomorphic to $C_{0}\left(X, \mathcal{E}_{H_{X}}\right) \otimes C_{0}\left(X, \mathcal{E}_{H_{X}}^{\mathrm{o}} \otimes \operatorname{Cliff}(T X)\right.$. Therefore

$\mathrm{KK}\left(C_{0}(X) \otimes C_{0}(X, \operatorname{Cliff}(T X), \mathbb{C}) \cong \mathrm{KK}\left(C_{0}\left(X, \mathcal{E}_{H_{X}}\right) \otimes C_{0}\left(X, \mathcal{E}_{H_{X}}^{\mathrm{o}} \otimes \operatorname{Cliff}(T X), \mathbb{C}\right)\right.\right.$, giving rise to a fundamental class in $\mathrm{KK}\left(C_{0}\left(X, \mathcal{E}_{H_{X}}\right) \otimes C_{0}\left(X, \mathcal{E}_{H_{X}}^{\mathrm{o}} \otimes \operatorname{Cliff}(T X), \mathbb{C}\right)\right.$, and showing that $\left(C_{0}\left(X, \mathcal{E}_{H_{X}}\right), C_{0}\left(X, \mathcal{E}_{H_{X}}^{\mathrm{o}} \otimes \operatorname{Cliff}(T X)\right)\right)$ is a strong PD pair. The analogous statement is true for $Y$. Finally, if $f^{*} \mathrm{~W}_{3}(Y)=\mathrm{W}_{3}(X)$, where $\mathrm{W}_{3}(X) \in \mathrm{H}^{3}(X, \mathbb{Z})$ is the third integral Stiefel-Whitney class of $X$, then we get the commutative diagram,

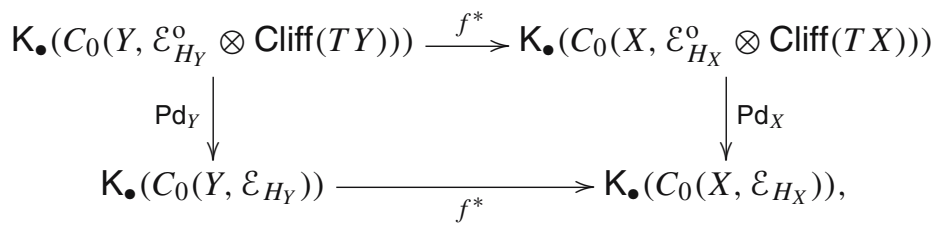

where the vertical arrows are isomorphisms. Then

$$
\left(f^{*}\right) ! \in \mathrm{KK}\left(C_{0}\left(X, \mathcal{E}_{H_{X}}\right), C_{0}\left(Y, \mathcal{E}_{H_{Y}}\right)\right)
$$

is defined as the Kasparov product $(-1)^{\operatorname{dim} Y} \Delta_{Y}^{\vee} \otimes\left[f^{*}\right] \otimes \Delta_{X}$.

This is a special case of the more general situation given as follows. Let $\left(\mathcal{A}_{i}, \mathcal{B}_{i}\right), i=$ 1, 2 be strong PD pairs with fundamental classes $\Delta_{i}, i=1,2$ respectively, and let $f: \mathcal{A}_{1} \rightarrow \mathcal{A}_{2}$ be a morphism. Then $f ! \in \mathrm{KK}\left(\mathcal{B}_{2}, \mathcal{B}_{1}\right)$ is defined using the diagram calculus in Appendix B as $(-1)^{d_{1}} \Delta_{1}^{\vee} \otimes_{\mathcal{A}_{1}}[f] \otimes_{\mathcal{A}_{2}} \Delta_{2}$.

There are also many interesting examples of strongly $\mathrm{K}$-oriented maps between noncommutative foliation $C^{*}$-algebras constructed in [42].

7.4. Weakly K-oriented maps. We will consider a subcategory $\underline{\mathfrak{C}}_{w}$ of the category of separable $C^{*}$-algebras and morphisms of $C^{*}$-algebras, consisting of "weakly K-oriented morphisms". $\underline{\mathfrak{C}}_{w}$ comes equipped with a contravariant functor $!: \underline{\mathfrak{C}}_{w} \rightarrow \underline{\mathrm{Ab}}(\underline{\mathrm{Ab}}$ denotes the category of $\mathbb{Z}_{2}$-graded abelian groups) sending

$$
\underline{\mathfrak{C}}_{w} \ni(\mathcal{A} \stackrel{f}{\longrightarrow} \mathcal{B}) \longrightarrow f_{!} \in \operatorname{Hom}_{\mathbb{Z}}\left(\mathrm{K}_{\bullet}(\mathcal{B}), \mathrm{K}_{\bullet}+d(\mathcal{A})\right)
$$

and having the following properties: 
(1) For any $C^{*}$-algebra $\mathcal{A}$, the identity morphism $\operatorname{id}_{\mathcal{A}}: \mathcal{A} \rightarrow \mathcal{A}$ is weakly K-oriented;

(2) If $(\mathcal{A} \stackrel{f}{\longrightarrow} \mathcal{B}) \in \underline{\mathfrak{C}}_{w}$, then $\left(\mathcal{A}^{\mathrm{o}} \stackrel{f^{\mathrm{o}}}{\longrightarrow} \mathcal{B}^{\mathrm{o}}\right) \in \underline{\mathfrak{C}}_{w}$ and moreover $\left(f_{!}\right)^{\mathrm{o}}=\left(f^{\mathrm{o}}\right)$ !;

(3) If $\mathcal{A}$ and $\mathcal{B}$ are weak PD algebras, then any morphism $(\mathcal{A} \stackrel{f}{\longrightarrow} \mathcal{B}) \in \underline{\mathfrak{C}}_{w}$, and $f$ ! is determined as follows:

$$
f_{!}=\mathrm{Pd}_{\mathcal{A}}^{-1} \circ\left(f^{\mathrm{o}}\right)^{*} \circ \mathrm{Pd}_{\mathcal{B}}
$$

where $\left(f^{\mathrm{o}}\right)^{*}$ denotes the morphism in $\operatorname{Hom}_{\mathbb{Z}}\left(\mathrm{K}^{\bullet}\left(\mathcal{A}^{\mathrm{o}}\right), \mathrm{K}^{\bullet}\left(\mathcal{B}^{\mathrm{o}}\right)\right)$.

This definition generalizes the one in the previous subsection in the following sense:

Proposition 7.8. $\underline{\mathfrak{C}}$ can be taken to be a subcategory of $\underline{\mathfrak{C}}_{w}$. In other words, if $f: \mathcal{A} \rightarrow \mathcal{B}$ is a morphism in $\underline{\mathfrak{C}}$, then $f_{!}=(f !)_{*}: \mathrm{K}_{\bullet}(\mathcal{B}) \rightarrow \mathrm{K}_{\bullet+d}(\mathcal{A})$ satisfies the above requirements.

Proof. Functoriality is obvious since we are merely composing the functor from $\mathfrak{C}$ to $\underline{\mathrm{KK}}$ with the functor from $\underline{\mathrm{KK}}$ to $\underline{\mathrm{Ab}}$ that sends $\mathcal{A} \mapsto \mathrm{K} \cdot(\mathcal{A}), \operatorname{KK}(\mathcal{A}, \mathcal{B}) \ni x \mapsto x_{*} \in$ $\operatorname{Hom}_{\mathbb{Z}}\left(\mathrm{K}_{\bullet}(\mathcal{A}), \mathrm{K}_{\bullet}(\mathcal{B})\right)$.

We need to check property (3). In other words, suppose $\mathcal{A}$ and $\mathcal{B}$ are strong PD algebras and $f ! \in \mathrm{KK}_{d}(\mathcal{B}, \mathcal{A})$ is defined to be $(-1)^{d_{\mathcal{A}}} \Delta_{\mathcal{A}}^{\vee} \otimes_{\mathcal{A}^{\circ}}\left[f^{\mathrm{o}}\right] \otimes_{\mathcal{B}^{\circ}} \Delta_{\mathcal{B}}$. We want to show that the induced map on $\mathrm{K}_{\bullet}$ is $\mathrm{Pd}_{\mathcal{A}}^{-1} \circ\left(f^{0}\right)^{*} \circ \mathrm{Pd}_{\mathcal{B}}$. However, this is obvious, since the Kasparov product with $\Delta_{\mathcal{B}} \in \mathrm{KK}_{d_{\mathcal{B}}}\left(\mathcal{B} \otimes \mathcal{B}^{\circ}, \mathbb{C}\right)$ is $\mathrm{Pd}_{\mathcal{B}}: \mathrm{K}_{\bullet}(\mathcal{B}) \rightarrow \mathrm{K}^{\bullet-} d_{\mathcal{B}}\left(\mathcal{B}^{\circ}\right)$ and the Kasparov product with $(-1)^{d_{\mathcal{A}}} \Delta_{\mathcal{A}}^{\vee} \in \mathrm{KK}_{d_{\mathcal{A}}}\left(\mathbb{C}, \mathcal{A} \otimes \mathcal{A}^{\mathrm{o}}\right)$ is $\operatorname{Pd}_{\mathcal{A}}^{-1}: \mathrm{K}^{\bullet}(\mathcal{A}) \rightarrow$ $\mathrm{K}_{\bullet+d_{\mathcal{A}}}\left(\mathcal{A}^{\mathrm{o}}\right)$.

Remark 7.9. The Gysin maps in $\mathrm{K}$-homology $f^{!} \in \operatorname{Hom}_{\mathbb{Z}}\left(\mathrm{K}^{\bullet}(\mathcal{A}), \mathrm{K}^{\bullet+d}(\mathcal{B})\right)$ can also be defined with completely analogous properties. There are also the obvious HL-theory analogues, used in the next subsection.

7.5. Grothendieck-Riemann-Roch formulas: the Strong case. The Grothendieck-Riemann-Roch formula compares the two bivariant cyclic classes $\operatorname{ch}(f !)$ and $f^{\mathrm{HL}}$ !.

Theorem 7.10. Suppose $\mathcal{A}$ and $\mathcal{B}$ are strong $P D$ algebras with given $\mathrm{HL}$ fundamental classes. Then one has the Grothendieck-Riemann-Roch formula,

$$
\operatorname{ch}(f !)=(-1)^{d_{\mathcal{B}}} \operatorname{Todd}(\mathcal{B}) \otimes_{\mathcal{B}} f^{\mathrm{HL}} ! \otimes_{\mathcal{A}} \operatorname{Todd}(\mathcal{A})^{-1} .
$$

Proof. We will write out the right-hand side of (7.18) and simplify. In the notation of Definition 7.2, the Todd class of $\mathcal{B}$ is the class

$$
\operatorname{Todd}(\mathcal{B})=\Xi_{\mathcal{B}}^{\vee} \otimes_{\tilde{\mathcal{B}}} \operatorname{ch}\left(\Delta_{\mathcal{B}}\right) \in \mathrm{HL}_{0}(\mathcal{B}, \mathcal{B}),
$$

and the inverse of the Todd class of $\mathcal{A}$ is the class

$$
\operatorname{Todd}(\mathcal{A})^{-1}=(-1)^{d_{\mathcal{A}}} \operatorname{ch}\left(\Delta_{\mathcal{A}}^{\vee}\right) \otimes_{\tilde{\mathcal{A}}} \Xi_{\mathcal{A}} \in \operatorname{HL}_{0}(\mathcal{A}, \mathcal{A}) .
$$

Since $\mathcal{A}$ and $\mathcal{B}$ are strong PD algebras, then $f^{\mathrm{HL}}$ ! is determined as follows:

$$
f^{\mathrm{HL}} !=(-1)^{d_{\mathcal{A}}} \Xi_{\mathcal{A}}^{\vee} \otimes_{\tilde{\mathcal{A}}}\left[\left(f^{\mathrm{HL}}\right)^{\mathrm{o}}\right] \otimes_{\tilde{\mathcal{B}}} \Xi_{\mathcal{B}},
$$


where $\left[f^{\mathrm{HL}}\right]=\mathrm{HL}(f)$ denotes the class in $\operatorname{HL}(\mathcal{A}, \mathcal{B})$ of the morphism $(\mathcal{A} \stackrel{f}{\longrightarrow} \mathcal{B})$ and $\left[\left(f^{\mathrm{HL}}\right)^{\mathrm{O}}\right]$ is defined similarly.

Therefore the right-hand side of (7.18) is equal to

$$
(-1)^{d_{\mathcal{B}}}\left(\Xi_{\mathcal{B}}^{\vee} \otimes_{\tilde{\mathcal{B}}} \operatorname{ch}\left(\Delta_{\mathcal{B}}\right)\right) \otimes_{\mathcal{B}}\left(\Xi_{\mathcal{A}}^{\vee} \otimes_{\tilde{\mathcal{A}}}\left[\left(f^{\mathrm{HL}}\right)^{\mathrm{o}}\right] \otimes_{\tilde{\mathcal{B}}} \Xi_{\mathcal{B}}\right) \otimes_{\mathcal{A}}\left(\operatorname{ch}\left(\Delta_{\mathcal{A}}^{\vee}\right) \otimes_{\tilde{\mathcal{A}}} \Xi_{\mathcal{A}}\right),
$$

which by the associativity of the intersection product, or equivalently by the diagram calculus of Appendix B (there it is worked out for $\mathrm{KK}$, but it works the same way for $\mathrm{HL}$ ), is equal to

$$
(-1)^{d_{\mathcal{B}}}\left(\Xi_{\mathcal{A}}^{\vee} \otimes_{\mathcal{A}}\left(\operatorname{ch}\left(\Delta_{\mathcal{A}}^{\vee}\right) \otimes_{\tilde{\mathcal{A}}} \Xi_{\mathcal{A}}\right)\right) \otimes_{\tilde{\mathcal{A}}}\left[\left(f^{\mathrm{HL}}\right)^{\mathrm{o}}\right] \otimes_{\tilde{\mathcal{B}}}\left(\left(\Xi_{\mathcal{B}}^{\vee} \otimes_{\tilde{\mathcal{B}}} \operatorname{ch}\left(\Delta_{\mathcal{B}}\right)\right) \otimes_{\mathcal{B}} \Xi_{\mathcal{B}}\right)
$$

On the other hand,

$$
f !=(-1)^{d_{\mathcal{A}}} \Delta_{\mathcal{A}}^{\vee} \otimes_{\tilde{\mathcal{A}}}\left[f^{\mathrm{o}}\right] \otimes_{\tilde{\mathcal{B}}} \Delta_{\mathcal{B}}
$$

Therefore the left-hand side of (7.18) is equal to

$$
(-1)^{d_{\mathcal{A}}} \operatorname{ch}\left(\Delta_{\mathcal{A}}^{\vee}\right) \otimes_{\tilde{\mathcal{A}}} \operatorname{ch}\left[f^{\mathrm{o}}\right] \otimes_{\tilde{\mathcal{B}}} \operatorname{ch}\left(\Delta_{\mathcal{B}}\right)
$$

By the functorial properties of the bivariant Chern character, one has

$$
\operatorname{ch}\left[f^{\mathrm{o}}\right]=\left[\left(f^{\mathrm{HL}}\right)^{\mathrm{o}}\right]
$$

In order to prove the theorem, it therefore suffices to prove that

$$
\left(\Xi_{\mathcal{B}}^{\vee} \otimes_{\tilde{\mathcal{B}}} \operatorname{ch}\left(\Delta_{\mathcal{B}}\right)\right) \otimes_{\mathcal{B}} \Xi_{\mathcal{B}}=(-1)^{d_{\mathcal{B}}} \operatorname{ch}\left(\Delta_{\mathcal{B}}\right)
$$

and

$$
\Xi_{\mathcal{A}}^{\vee} \otimes_{\mathcal{A}}\left(\operatorname{ch}\left(\Delta_{\mathcal{A}}^{\vee}\right) \otimes_{\tilde{\mathcal{A}}} \Xi_{\mathcal{A}}\right)=(-1)^{d_{\mathcal{A}}} \operatorname{ch}\left(\Delta_{\mathcal{A}}^{\vee}\right)
$$

But both of these equalities also follow easily from the diagram calculus:

$$
\begin{aligned}
\left(\Xi_{\mathcal{B}}^{\vee} \otimes_{\tilde{\mathcal{B}}} \operatorname{ch}\left(\Delta_{\mathcal{B}}\right)\right) \otimes_{\mathcal{B}} \Xi_{\mathcal{B}} & =\left(\Xi_{\mathcal{B}}^{\vee} \otimes_{\mathcal{B}} \Xi_{\mathcal{B}}\right) \otimes_{\tilde{\mathcal{B}}} \operatorname{ch}\left(\Delta_{\mathcal{B}}\right) \\
& =(-1)^{d_{\mathcal{B}}} 1_{\tilde{\mathcal{B}}} \otimes_{\tilde{\mathcal{B}}} \operatorname{ch}\left(\Delta_{\mathcal{B}}\right) \\
& =(-1)^{d_{\mathcal{B}}} \operatorname{ch}\left(\Delta_{\mathcal{B}}\right)
\end{aligned}
$$

and

$$
\begin{aligned}
\Xi_{\mathcal{A}}^{\vee} \otimes_{\mathcal{A}}\left(\operatorname{ch}\left(\Delta_{\mathcal{A}}^{\vee}\right) \otimes_{\tilde{\mathcal{A}}} \Xi_{\mathcal{A}}\right) & =\operatorname{ch}\left(\Delta_{\mathcal{A}}^{\vee}\right) \otimes_{\tilde{\mathcal{A}}}\left(\Xi_{\mathcal{A}}^{\vee} \otimes_{\mathcal{A}} \Xi_{\mathcal{A}}\right) \\
& =\operatorname{ch}\left(\Delta_{\mathcal{A}}^{\vee}\right) \otimes_{\tilde{\mathcal{A}}}(-1)^{d_{\mathcal{A}}} 1_{\tilde{\mathcal{A}}} \\
& =(-1)^{d_{\mathcal{A}}} \operatorname{ch}\left(\Delta_{\mathcal{A}}^{\vee}\right) .
\end{aligned}
$$


7.6. Grothendieck-Riemann-Roch formulas: the Weak case. For $(\mathcal{A} \stackrel{f}{\longrightarrow} \mathcal{B})$, weakly $\mathrm{K}$-oriented, the Grothendieck-Riemann-Roch formula repairs the noncommutativity of the diagram,

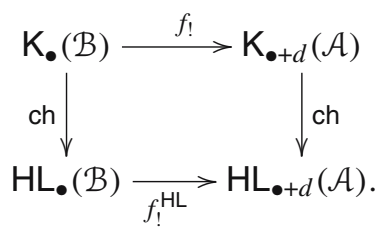

The following can be proved in an analogous way to the strong case of the GrothendieckRiemann-Roch formula, Theorem 7.10, so we will omit the proof.

Theorem 7.11. If $f: \mathcal{A} \rightarrow \mathcal{B}$ is a morphism in $\underline{\mathfrak{C}}_{w}$, and $\xi \in \mathrm{K}_{\bullet}(\mathcal{B})$, then one has the Grothendieck-Riemann-Roch formula,

$$
\operatorname{ch}(f ! \xi) \otimes_{\mathcal{A}} \operatorname{Todd}(\mathcal{A})=(-1)^{d_{\mathcal{B}}} f_{!}^{\mathrm{HL}}\left(\operatorname{ch}(\xi) \otimes_{\mathcal{B}} \operatorname{Todd}(\mathcal{B})\right)
$$

Remark 7.12. Let $\mathcal{A}$ be a unital PD algebra having an even degree fundamental class in K-theory. Then there is a canonical morphism, $\lambda: \mathbb{C} \rightarrow \mathcal{A}$, given by $\mathbb{C} \ni z \mapsto z \cdot 1 \in \mathcal{A}$, where 1 denotes the unit in $\mathcal{A}$. Observe that $\lambda$ is always weakly $\mathrm{K}$-oriented, since $\mathbb{C}$ is a $\mathrm{PD}$ algebra, and the Gysin map $\lambda_{!}: \mathrm{K}_{0}(\mathcal{A}) \rightarrow \mathbb{Z}$ is the analog of the topological index morphism (for compact manifolds). Theorem 7.11 above applied to this situation says that,

$$
\lambda_{!}(\xi)=\lambda_{!}^{\mathrm{HL}}\left(\operatorname{ch}(\xi) \otimes_{\mathcal{A}} \operatorname{Todd}(\mathcal{A})\right)
$$

where $\lambda_{!}^{\mathrm{HL}}: \mathrm{HL}_{0}(\mathcal{A}) \rightarrow \mathbb{C}$ is the associated Gysin morphism in cyclic theory. In the case where $\mathcal{A}=C(X), X$ a compact $\operatorname{spin}^{c}$ manifold, this is just the usual Atiyah-Singer index theorem. Indeed, $\lambda_{!}(\xi)=\operatorname{index} \operatorname{Pd}_{X}(\xi)=\operatorname{index}\left(D_{\xi}\right)$, while the other side of the index formula is $\lambda_{1}^{\mathrm{HL}}\left(\operatorname{ch}(\xi) \otimes_{\mathcal{A}} \operatorname{Todd}(\mathcal{A})\right)=(\operatorname{Todd}(X) \cup \operatorname{ch}(\xi))[X]$. Note in particular that when $\xi$ is the canonical rank one free module over $\mathcal{A}$, then we obtain a numerical invariant which we call the Todd genus of $\mathcal{A}$, a characteristic number of the algebra.

\section{Noncommutative D-Brane Charges}

In this final section we will come to the main motivation for the present work, the "Dbrane charge formula" for very general noncommutative spacetimes. The crux of the definition of D-brane charge in Sect. 1 relied upon the introduction of natural pairings in K-theory and singular cohomology, which in turn arose as a consequence of Poincaré duality. We are now ready to describe the analogs of the natural pairings in appropriate noncommutative cases. The key point is that the multiplication map $m: \mathcal{A} \otimes \mathcal{A} \rightarrow \mathcal{A}$ is an algebra homomorphism only in the commutative case and one needs to replace its role with some new construct. This is where the formalism of KK-theory plays a crucial role. Mathematically, the problem is concerned with taking the square root of the Todd class of a noncommutative spacetime, under mild hypotheses. This then enables one to "correct" the Chern character so that the index pairing in K-theory and the given pairing in HL-theory agree. 
8.1. Poincaré pairings. In the notation of Sect. 7.1, let $\mathcal{A} \in \underline{D}$ and $\alpha \in \mathrm{K}_{i}(\mathcal{A})$, $\beta \in \mathrm{K}_{-d-i}(\tilde{\mathcal{A}})$. Then there is a pairing

$$
(\alpha, \beta) \longmapsto\langle\alpha, \beta\rangle=(\alpha \times \beta) \otimes_{\mathcal{A} \otimes \tilde{\mathcal{A}}} \Delta \in \mathrm{KK}_{0}(\mathbb{C}, \mathbb{C})=\mathbb{Z}
$$

In the case where $\mathcal{A}=\tilde{\mathcal{A}}=C(X)$ is the algebra of continuous functions on a $\operatorname{spin}^{c}$ manifold and the fundamental class $\Delta$ comes from the Dirac operator, this is the same as the pairing (1.1) introduced earlier, and is the K-theory analogue of the cup-product pairing (1.5). Indeed, in this case,

$$
\langle\alpha, \beta\rangle=\operatorname{Pd}_{X}(\alpha) \otimes_{C(X)} \beta=\not_{\alpha} \otimes_{C(X)} \beta=\operatorname{index}\left(\not D_{\alpha \otimes \beta}\right) .
$$

If $\mathcal{A}$ and $\tilde{\mathcal{A}}$ have finitely generated K-theory and satisfy the Universal Coefficient Theorem (UCT), then the pairing (8.1) is nondegenerate modulo torsion.

In the case of a strong PD algebra, since we have $\tilde{\mathcal{A}}=\mathcal{A}^{\mathrm{o}}$, whose K-theory is canonically isomorphic to that of $\mathcal{A}$ itself (by Remark 2.19), the pairing (8.1) can be viewed as a pairing of $\mathrm{K}_{\bullet}(\mathcal{A})$ with itself. Then we are led to consider the following additional condition.

Definition 8.1. A fundamental class $\Delta$ of a strong $P D$ algebra $\mathcal{A}$ is said to be symmetric if $\sigma(\Delta)^{\mathrm{o}}=\Delta \in \mathrm{K}^{d}\left(\mathcal{A} \otimes \mathcal{A}^{\mathrm{o}}\right)$, where

$$
\sigma: \mathcal{A} \otimes \mathcal{A}^{\mathrm{o}} \longrightarrow \mathcal{A}^{\mathrm{o}} \otimes \mathcal{A}
$$

is the involution $x \otimes y^{\mathrm{o}} \mapsto y^{\mathrm{o}} \otimes x$ and $\sigma$ also denotes the induced map on $\mathrm{K}$-homology. In terms of the diagram calculus of Appendix $B, \Delta$ being symmetric implies that

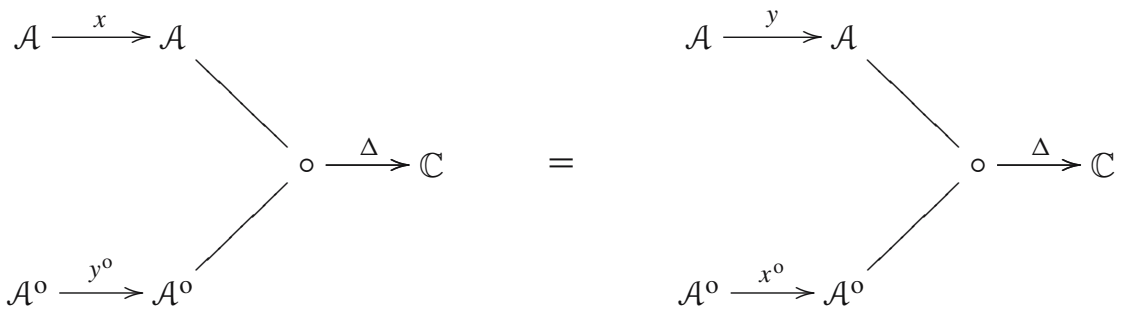

for all $x$ and $y$.

Symmetry is a natural condition to consider, since the intersection pairing on an even-dimensional manifold is symmetric.

Proposition 8.2. For any strong Poincaré duality algebra $\mathcal{A}$ there exists a bilinear pairing on K-theory:

$$
\langle-,-\rangle: \mathrm{K}_{i}(\mathcal{A}) \times \mathrm{K}_{d-i}(\mathcal{A}) \longrightarrow \mathbb{Z}
$$

defined by

$$
\langle\alpha, \beta\rangle=\left(\alpha \times \beta^{\mathrm{o}}\right) \otimes_{\mathcal{A} \otimes \mathcal{A}^{\mathrm{o}}} \Delta \in \mathrm{KK}_{0}(\mathbb{C}, \mathbb{C})=\mathbb{Z} .
$$

Moreover, if the fundamental class $\Delta$ is symmetric, then the bilinear pairing (8.3) on $\mathrm{K}$-theory is symmetric. If $\mathcal{A}$ satisfies the UCT in $\mathrm{K}$-theory and has finitely generated $\mathrm{K}$-theory, then the pairing (8.3) is nondegenerate modulo torsion. 
Proof. Immediate from the remarks above.

If $\mathcal{A}$ is a strong C-PD algebra, then the local cyclic homology and cohomology of $\mathcal{A}$ are isomorphic. This is equivalent to saying that the canonical pairing

$$
(-,-): \mathrm{HL}_{i}(\mathcal{A}) \otimes_{\mathbb{C}} \mathrm{HL}_{d-i}(\mathcal{A}) \longrightarrow \mathbb{C}
$$

on cyclic homology, given by

$$
(x, y)=\left(x \times y^{\mathrm{o}}\right) \otimes_{\mathcal{A} \otimes \mathcal{A}^{\mathrm{o}}} \Xi
$$

for $x \in \mathrm{HL}_{i}(\mathcal{A})$ and $y \in \mathrm{HL}_{d-i}(\mathcal{A})$, is non-degenerate, since the pairing between $\mathrm{HL}_{\bullet}(\mathcal{A})$ and $\mathrm{HL}^{\bullet}(\mathcal{A})$ is always non-degenerate for any algebra, at least if the universal coefficient theorem holds. In the commutative case, this pairing coincides with the intersection form (1.5).

If $\mathcal{A}$ is a strong PD algebra, then one can also define a bilinear form on cyclic homology determined by the class $\operatorname{ch}(\Delta)$ as

$$
(-,-)_{\mathfrak{h}}: \mathrm{HL}_{i}(\mathcal{A}) \otimes_{\mathbb{C}} \mathrm{HL}_{d-i}(\mathcal{A}) \longrightarrow \mathbb{C}
$$

by setting

$$
(x, y)_{\mathfrak{h}}=\left(x \times y^{\mathrm{o}}\right) \otimes_{\mathcal{A} \otimes \mathcal{A}^{\circ}} \operatorname{ch}(\Delta)
$$

for $x \in \mathrm{HL}_{i}(\mathcal{A})$ and $y \in \mathrm{HL}_{d-i}(\mathcal{A})$.

A fundamental class in $\mathrm{HL}$-theory is said to be symmetric if $\sigma(\Xi)^{\mathrm{o}}=\Xi \in \mathrm{HL}^{d}(\mathcal{A} \otimes$ $\mathcal{A}^{\mathrm{o}}$ ), where $\sigma$ is the involution defined earlier in (8.2) and $\sigma$ also denotes the induced map on HL-theory.

8.2. D-Brane charge formula for noncommutative spacetimes. If $\mathcal{A}$ is a strong PD algebra, then we have defined in the previous subsection two pairings, one given by the formula (8.7), and the other by the formula (8.5). These two pairings will a priori be different. Comparing them is the crux of our definition of D-brane charge. Let us begin with the following observation.

Proposition 8.3. If $\mathcal{A}$ is a strong PD algebra, then the Chern character $\mathrm{ch}: \mathrm{K}_{\mathbf{\bullet}}(\mathcal{A}) \rightarrow$ $\mathrm{HL}_{\bullet}(\mathcal{A})$ is an isometry with respect to the inner products given in Eqs. (8.3) and (8.7),

$$
\langle p, q\rangle=(\operatorname{ch}(p), \operatorname{ch}(q))_{\mathfrak{h}} \cdot
$$

Proof. Using multiplicativity of the Chern character, one has

$$
(\operatorname{ch}(p), \operatorname{ch}(q))_{\mathfrak{h}}=\operatorname{ch}\left(\left(p \times q^{\mathrm{o}}\right) \otimes_{\mathcal{A} \otimes \mathcal{A}^{\circ}} \Delta\right) .
$$

Now use the fact that ch is a unital homomorphism (this is essentially the index theorem, i.e., the statement that the index pairing (8.3) coincides with the canonical pairing between the corresponding Chern characters in local cyclic homology). 
From this proposition it follows that the bilinear form (8.7) is the analogue of the twisted inner product defined in (1.11). Finding the appropriate modified Chern character which maps (8.5) onto (8.7) will thereby yield the formula for D-brane charge that we are looking for.

The technical problem that we are faced with is to take the square root of the Todd class $\operatorname{Todd}(\mathcal{A})$ in $\operatorname{HL}(\mathcal{A}, \mathcal{A})$. To do this, we will assume that the Universal Coefficient Theorem holds for the noncommutative spacetime $\mathcal{A}$. $\operatorname{Then} \operatorname{HL}(\mathcal{A}, \mathcal{A})=\operatorname{End}(\mathrm{HL} \bullet(\mathcal{A}))$. In addition, we will assume that $\operatorname{dim}_{\mathbb{C}} \mathrm{HL}_{\bullet}(\mathcal{A})$ is finite, say equal to $n$. Then since $\operatorname{Todd}(\mathcal{A})$ is in $G L\left(\mathrm{HL}_{\bullet}(\mathcal{A})\right) \cong G L_{n}(\mathbb{C})$ and every matrix in $G L_{n}(\mathbb{C})$ has a square root (use the Jordan canonical form to prove this!), we can take a square root, $\sqrt{\operatorname{Todd}(\mathcal{A})}$. Using the $\mathrm{UCT}, \sqrt{\operatorname{Todd}(\mathcal{A})}$ can again be considered as an element in $\mathrm{HL}(\mathcal{A}, \mathcal{A})$. The square root is not unique, but we fix a choice. In some cases, the Todd class may be self-adjoint and positive with respect to a suitable inner product on $\mathrm{HL}_{\bullet}(\mathcal{A})$, which might help to pin down a more canonical choice. In any event, we have the following theorem.

Theorem 8.4 (Isometric pairing formula). Suppose that the noncommutative spacetime $\mathcal{A}$ satisfies the UCT for local cyclic homology, and that $\mathrm{HL}_{\bullet}(\mathcal{A})$ is a finite dimensional vector space. If $\mathcal{A}$ has symmetric (even-dimensional) fundamental classes both in $\mathrm{K}$-theory and in cyclic theory, then the modified Chern character

$$
\operatorname{ch} \otimes_{\mathcal{A}} \sqrt{\operatorname{Todd}(\mathcal{A})}: \mathrm{K}_{\bullet}(\mathcal{A}) \rightarrow \mathrm{HL}_{\bullet}(\mathcal{A})
$$

is an isometry with respect to the inner products (8.1) and (8.5),

$$
\langle p, q\rangle=\left(\operatorname{ch}(p) \otimes_{\mathcal{A}} \sqrt{\operatorname{Todd}(\mathcal{A})}, \operatorname{ch}(q) \otimes_{\mathcal{A}} \sqrt{\operatorname{Todd}(\mathcal{A})}\right) .
$$

Proof. To prove the theorem, we use Proposition 8.3 and observe that it's enough to show that the right-hand sides of Eqs. (8.11) and (8.9) agree. For this we use the diagram calculus of Appendix B,
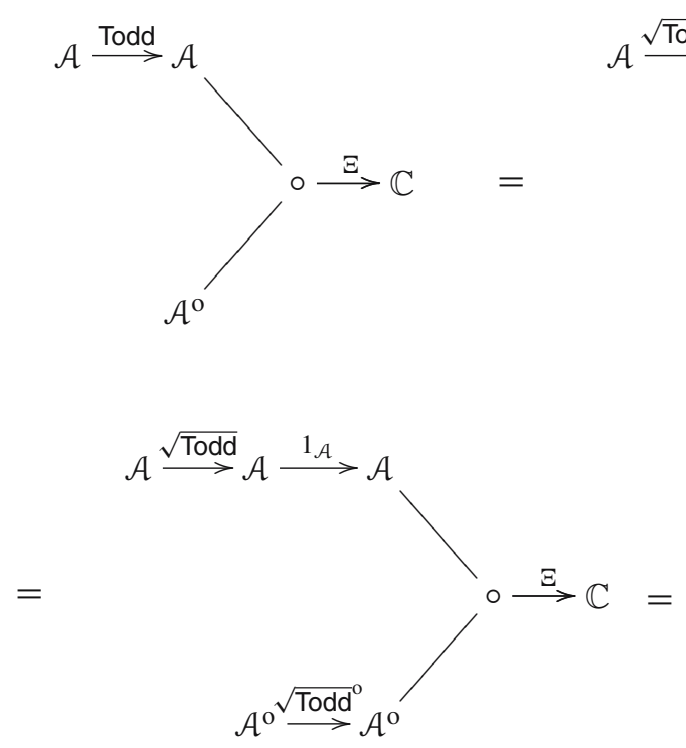
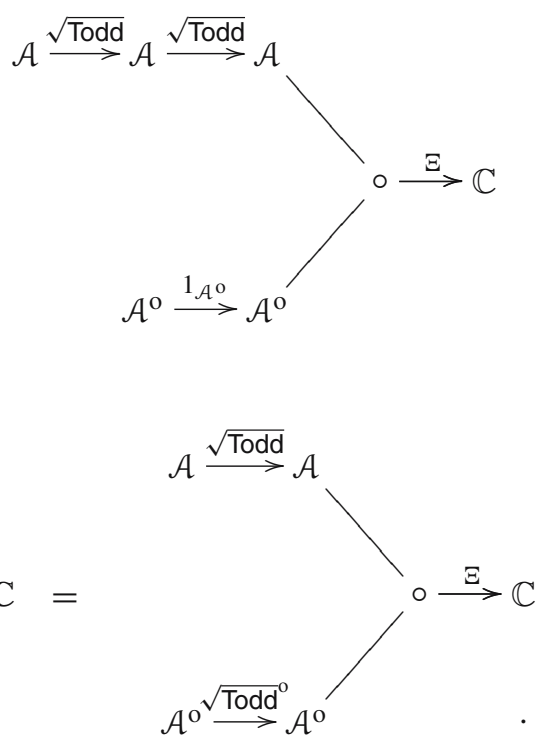
Note that the symmetry of $\Xi$ is used here in a crucial way. This computation shows that

$$
\begin{aligned}
& \left(\operatorname{ch}(p) \otimes_{\mathcal{A}} \sqrt{\operatorname{Todd}}(\mathcal{A}), \operatorname{ch}(p) \otimes_{\mathcal{A}} \sqrt{\operatorname{Todd}}(\mathcal{A})\right) \\
& =(\operatorname{ch}(p) \otimes \operatorname{ch}(q)) \otimes_{\mathcal{A} \otimes \mathcal{A}^{o}}\left(\operatorname{Todd}(\mathcal{A}) \otimes_{\mathcal{A}} \Xi\right) .
\end{aligned}
$$

Since, by definition, $\operatorname{Todd}(\mathcal{A})=\Xi^{\vee} \otimes_{\mathcal{A}^{o}} \operatorname{ch}(\Delta)$, a similar computation shows that $\operatorname{Todd}(\mathcal{A}) \otimes_{\mathcal{A}} \Xi=\left(\Xi^{\vee} \otimes_{\mathcal{A}} \Xi\right) \otimes_{\mathcal{A}^{o}} \operatorname{ch}(\Delta)=\operatorname{ch}(\Delta)$ and so

$$
\begin{aligned}
\left(\operatorname{ch}(p) \otimes_{\mathcal{A}} \sqrt{\operatorname{Todd}}(\mathcal{A}), \operatorname{ch}(p) \otimes_{\mathcal{A}} \sqrt{\operatorname{Todd}}(\mathcal{A})\right) & =(\operatorname{ch}(p) \otimes \operatorname{ch}(q)) \otimes_{\mathcal{A} \otimes \mathcal{A}^{o}} \operatorname{ch}(\Delta) \\
& =\operatorname{ch}\left((p \otimes q) \otimes_{\left.\mathcal{A} \otimes \mathcal{A}^{o} \Delta\right)}\right. \\
& =\operatorname{ch}((p, q)) .
\end{aligned}
$$

Finally, the Chern character $\mathbb{Z}=\mathrm{KK}(\mathbb{C}, \mathbb{C}) \rightarrow \mathrm{HL}(\mathbb{C}, \mathbb{C})=\mathbb{C}$ is injective, which gives the desired result.

Corollary 8.5 (D-brane charge formula for noncommutative spacetimes). Suppose that the noncommutative spacetime $\mathcal{A}$ satisfies the hypotheses of Theorem 8.4 above. Then there is a noncommutative analogue of the well-known formula (1.1) in [63] for the charge associated to a $D$-brane $\mathcal{B}$ in a noncommutative spacetime $\mathcal{A}$ with given weakly K-oriented morphism $f: \mathcal{A} \rightarrow \mathcal{B}$ and Chan-Paton bundle $\xi \in \mathrm{K}_{\bullet}(\mathcal{B})$,

$$
\mathrm{Q}_{\xi}=\operatorname{ch}\left(f_{!}(\xi)\right) \otimes_{\mathcal{A}} \sqrt{\operatorname{Todd}(\mathcal{A})} .
$$

This is still not quite the most general situation. Corollary 8.5 deals with charges coming from a (weakly) K-oriented morphism $f: \mathcal{A} \rightarrow \mathcal{B}$, when a Chan-Paton bundle, i.e., a K-theory class, is given on $\mathcal{B}$. This is the obvious translation of the situation coming from a flat D-brane in the commutative case, but one can imagine more general noncommutative $\mathrm{D}$-branes, where the algebra $\mathcal{B}$ is missing, i.e., one simply has a Fredholm module for $\mathcal{A}$ representing a class in $\mathrm{K}^{\bullet}(\mathcal{A})$. (In Corollary 8.5, the associated class in $\mathrm{K}^{\bullet}(\mathcal{A})$ is $\operatorname{Pd}_{\mathcal{A}}\left(f_{!}(\xi)\right)=f^{*}\left(\operatorname{Pd}_{\mathcal{B}}(\xi)\right)$.) The final version of the charge formula is the following:

Proposition 8.6 (D-brane charge formula, dual version). Suppose that the noncommutative spacetime $\mathcal{A}$ satisfies the hypotheses of Theorem 8.4 above. Then there is a noncommutative analogue of formula (1.30) of Proposition 1.8 above for the dual charge associated to a D-brane in the noncommutative spacetime $\mathcal{A}$ represented by a class $\mu \in \mathrm{K}^{\bullet}(\mathcal{A})$ :

$$
\mathrm{Q}_{\mu}=\sqrt{\operatorname{Todd}(\mathcal{A})}^{-1} \otimes_{\mathcal{A}} \operatorname{ch}(\mu) .
$$

This formula satisfies the isometry rule:

$$
\Xi^{\vee} \otimes_{\mathcal{A} \otimes \mathcal{A}^{\mathrm{o}}}\left(\mathrm{Q}_{\mu} \times \mathrm{Q}_{\nu}^{\mathrm{o}}\right)=\Delta^{\vee} \otimes_{\mathcal{A} \otimes \mathcal{A}^{\mathrm{o}}}\left(\mu \times v^{\mathrm{o}}\right) .
$$

Proof. We need to check (8.14). By multiplicativity of the Chern character, the righthand side is equal to $\operatorname{ch}\left(\Delta^{\vee}\right) \otimes_{\mathcal{A} \otimes \mathcal{A}^{\circ}}\left(\operatorname{ch}(\mu) \times \operatorname{ch}(v)^{\mathrm{o}}\right)$. The left-hand side is

$$
\begin{aligned}
\Xi^{\vee} & \otimes_{\mathcal{A} \otimes \mathcal{A}^{\circ}}\left(Q_{\mu} \times Q_{\nu}^{o}\right) \\
= & \Xi^{\vee} \otimes_{\mathcal{A} \otimes \mathcal{A}^{\mathrm{o}}}\left(\sqrt{\operatorname{Todd}(\mathcal{A})}^{-1} \times{\sqrt{\operatorname{Todd}(\mathcal{A})^{\mathrm{o}}}}^{-1}\right) \otimes_{\mathcal{A} \otimes \mathcal{A}^{\mathrm{o}}}\left(\operatorname{ch}(\mu) \times \operatorname{ch}(\nu)^{\mathrm{o}}\right) \\
= & \text { (by symmetry of } \left.\Xi^{\vee} \text { as in the proof of Theorem } 8.4\right) \\
& \Xi^{\vee} \otimes_{\mathcal{A} \otimes \mathcal{A}^{\mathrm{o}}}\left(\operatorname{Todd}(\mathcal{A})^{-1} \times 1_{\mathcal{A}^{\mathrm{o}}}\right) \otimes_{\mathcal{A} \otimes \mathcal{A}^{\mathrm{o}}}\left(\operatorname{ch}(\mu) \times \operatorname{ch}(\nu)^{\mathrm{o}}\right) .
\end{aligned}
$$


But $\operatorname{ch}\left(\Delta^{\vee}\right)=\Xi^{\vee} \otimes_{\mathcal{A}} \operatorname{Todd}(\mathcal{A})^{-1}$, since (by the diagram calculus) $\Xi^{\vee} \otimes_{\mathcal{A}} \operatorname{Todd}(\mathcal{A})^{-1}=\Xi^{\vee} \otimes_{\mathcal{A}}\left(\operatorname{ch}\left(\Delta^{\vee}\right) \otimes_{\mathcal{A}^{\mathrm{o}}} \Xi\right)=\operatorname{ch}\left(\Delta^{\vee}\right) \otimes_{\mathcal{A}^{\mathrm{o}}}\left(\Xi^{\vee} \otimes_{\mathcal{A}} \Xi\right)=\operatorname{ch}\left(\Delta^{\vee}\right)$.

Remark 8.7. Although our noncommutative formulas for D-brane charges have been derived under the assumption that $\mathcal{A}$ is a strong PD algebra, they hold more generally for any algebra $\mathcal{A}$ belonging to the class $\mathfrak{D}$ introduced in Sect. 7.1. This allows us to write down charge formulas in a variety of very general situations. For instance, one can in this way obtain a bilinear pairing on twisted $\mathrm{K}$-theory, $\mathrm{K}^{\bullet}(X, H) \times \mathrm{K}^{\bullet}(X,-H) \rightarrow \mathbb{Z}$, and hence an isometric pairing between twisted K-theory and twisted cohomology, recovering the charge formula (1.13) of [8] for twisted D-branes.

\section{Appendix A. The Kasparov Product}

In this appendix, we will summarize the main properties of the intersection product. If $\mathcal{A}$ is a separable algebra then the exterior (or cup) product exists and defines a bilinear pairing, [53, Thm 2.11]:

$$
\mathrm{KK}_{i}\left(\mathcal{A}, \mathcal{B}_{1}\right) \otimes_{\mathcal{B}_{1}} \mathrm{KK}_{j}\left(\mathcal{B}_{1}, \mathcal{B}_{2}\right) \rightarrow \mathrm{KK}_{i+j}\left(\mathcal{A}, \mathcal{B}_{2}\right)
$$

In [53, Def. 2.12], Kasparov also defines the intersection product (which he calls the cap-cup product)

$$
\mathrm{KK}_{i}\left(\mathcal{A}_{1}, \mathcal{B}_{1} \otimes \mathcal{D}\right) \otimes_{\mathcal{D}} \mathrm{KK}_{j}\left(\mathcal{D} \otimes \mathcal{A}_{2}, \mathcal{B}_{2}\right) \rightarrow \mathrm{KK}_{i+j}\left(\mathcal{A}_{1} \otimes \mathcal{A}_{2}, \mathcal{B}_{1} \otimes \mathcal{B}_{2}\right)
$$

by the formula

$$
x_{1} \otimes_{\mathcal{D}} x_{2}=\left(x_{1} \otimes 1_{\mathcal{A}_{2}}\right) \otimes_{\mathcal{B}_{1} \otimes \mathcal{D} \otimes \mathcal{A}_{2}}\left(x_{2} \otimes 1_{\mathcal{B}_{1}}\right) .
$$

The exterior (or cup) product is obtained when $\mathcal{D}=\mathbb{C}$. This exterior product has the following properties [53, Thm. 2.14].

Theorem A.1. Let $\mathcal{A}_{1}$ and $\mathcal{A}_{2}$ be separable algebras. Then the intersection (cup-cap) product exists and is:

(1) bilinear;

(2) contravariant in $\mathcal{A}_{1}$ and $\mathcal{A}_{2}$;

(3) covariant in $\mathcal{B}_{1}$ and $\mathcal{B}_{2}$;

(4) functorial in $\mathcal{D}:$ for any morphism $f: \mathcal{D}_{1} \rightarrow \mathcal{C}$ one has

$$
f\left(x_{1}\right) \otimes_{\mathcal{D}_{2}} x_{2}=x_{1} \otimes_{\mathcal{D}_{1}} f\left(x_{2}\right) ;
$$

(5) associative: for any $x_{1} \in \mathrm{KK}_{i}\left(\mathcal{A}_{1}, \mathcal{B}_{1} \otimes \mathcal{D}_{1}\right), x_{2} \in \mathrm{KK}_{j}\left(\mathcal{D}_{1} \otimes \mathcal{A}_{2}, \mathcal{B}_{2} \otimes \mathcal{D}_{2}\right)$ and $x_{3} \in \mathrm{KK}_{k}\left(\mathcal{D}_{2} \otimes \mathcal{A}_{3}, \mathcal{B}_{3}\right)$, where $\mathcal{A}_{1}, \mathcal{A}_{2}, \mathcal{A}_{3}$ and $\mathcal{D}_{1}$ are assumed separable, the following formula holds:

$$
\left(x_{1} \otimes_{\mathcal{D}_{1}} x_{2}\right) \otimes_{\mathcal{D}_{2}} x_{3}=x_{1} \otimes_{\mathcal{D}_{1}}\left(x_{2} \otimes_{\mathcal{D}_{2}} x_{3}\right)
$$

(6) For any $x_{1} \in \mathrm{KK}_{i}\left(\mathcal{A}_{1}, \mathcal{B}_{1} \otimes \mathcal{D}_{1} \otimes \mathcal{D}\right), x_{2} \in \mathrm{KK}_{j}\left(\mathcal{D} \otimes \mathcal{D}_{2} \otimes \mathcal{A}_{2}, \mathcal{B}_{2}\right)$, where $\mathcal{A}_{1}, \mathcal{A}_{2}, \mathcal{D}_{2}$ are separable and $\mathcal{D}_{1}$ is $\sigma$-unital, the following formula holds:

$$
x_{1} \otimes_{\mathcal{D}} x_{2}=\left(x_{1} \otimes 1_{\mathcal{D}_{2}}\right) \otimes_{\mathcal{D}_{1} \otimes \mathcal{D} \otimes \mathcal{D}_{2}}\left(1_{\mathcal{D}_{1}} \otimes x_{2}\right)
$$




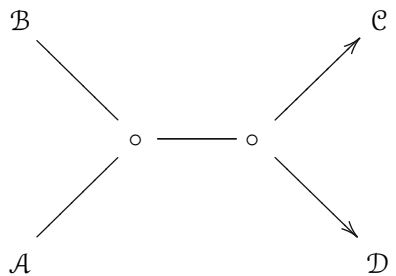

Fig. B.1. Diagram representing an element of $\operatorname{KK}(\mathcal{B} \otimes \mathcal{A}, \mathcal{C} \otimes \mathcal{D})$

(7) For $x_{1} \in \mathrm{KK}_{i}\left(\mathcal{A}_{1}, \mathcal{B}_{1} \otimes \mathcal{D}\right), x_{2} \in \mathrm{KK}_{j}\left(\mathcal{D} \otimes \mathcal{A}_{2}, \mathcal{B}_{2}\right)$, for separable algebras $\mathcal{A}_{1}, \mathcal{A}_{2}$ and $\mathcal{D}$, the following formula holds:

$$
\left(x_{1} \otimes_{\mathcal{D}} x_{2}\right) \otimes_{\mathbb{C}} 1_{\mathcal{D}_{1}}=\left(x_{1} \otimes 1_{\mathcal{D}_{1}}\right) \otimes{\mathcal{D} \otimes \mathcal{D}_{1}}\left(x_{2} \otimes 1_{\mathcal{D}_{1}}\right) ;
$$

(8) the cup product is commutative (over $\mathbb{C})$ :

$$
x_{1} \otimes_{\mathbb{C}} x_{2}=x_{2} \otimes_{\mathbb{C}} x_{1}
$$

and

(9) the element $1_{\mathbb{C}} \in \mathrm{KK}_{0}(\mathbb{C}, \mathbb{C})$ is a unit for this product:

$$
1_{\mathbb{C}} \otimes_{\mathbb{C}} x=x \otimes_{\mathbb{C}} 1_{\mathbb{C}}=x
$$

for all $x \in \mathrm{KK}_{i}(\mathcal{A}, \mathcal{B})$, where $\mathcal{A}$ is assumed to be separable.

\section{Appendix B. A Diagram Calculus for the Kasparov Product}

Keeping track of Kasparov products and the associativity formulae in the general case described above in Appendix A can be quite complicated. In this appendix we describe a pictorial calculus for keeping track of these things, which one of us (J.R.) has often found useful as a guide to calculations. In this appendix we will not write degree labels explicitly on KK-groups for the sake of notational convenience - in the most important case, all elements lie in $\mathrm{KK}_{0}$ anyway.

The idea is to represent an element of a KK group by a diagram (which we read from left to right), with one "input" for each tensor factor in the first argument of KK, and one "output" for each tensor factor in the second argument of KK. For convenience, we can also add arrowheads pointing toward the outputs. Thus, for example, an element of $\mathrm{KK}(\mathcal{B} \otimes \mathcal{A}, \mathcal{C} \otimes \mathcal{D})$ would be represented by a diagram like the one in Fig. B.1. Note that an element of $\mathrm{KK}(\mathcal{A} \otimes \mathcal{B}, \mathcal{C} \otimes \mathcal{D})$ would be represented by an almost identical diagram, having the two input terminals switched. The basic rule is that permutation of the input or output terminals may involve at most the switch of a sign.

The Kasparov product corresponds to concatenation of diagrams, except that one is only allowed to attach an input to a matching output. For example, in Fig. B.1, there are input terminals corresponding to both $\mathcal{B}$ and $\mathcal{A}$, so one can take the product over a Kasparov class having a $\mathcal{B}$ or $\mathcal{A}$ as an output terminal. For example, a class in $\operatorname{KK}(\mathcal{E}, \mathcal{A})$ would be represented by a diagram like Fig. B.2, and we can concatenate the diagrams as shown in Fig. B. 3 to obtain the product (over $\mathcal{A})$ in $\mathrm{KK}(\mathcal{B} \otimes \mathcal{E}, \mathcal{C} \otimes \mathcal{D}$ ) shown in Fig. B.4. 


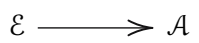

Fig. B.2. Diagram representing an element of $\operatorname{KK}(\mathcal{E}, \mathcal{A})$

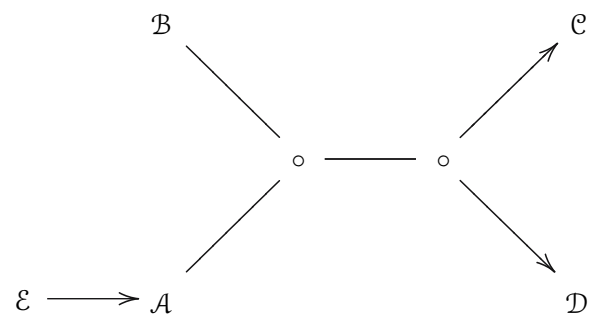

Fig. B.3. Diagram representing the intersection product, $\otimes_{\mathcal{A}}: \mathrm{KK}(\mathcal{E}, \mathcal{A}) \otimes \mathrm{KK}(\mathcal{B} \otimes \mathcal{A}, \mathcal{C} \otimes \mathcal{D}) \rightarrow \mathrm{KK}(\mathcal{B} \otimes$ $\mathcal{E}, \mathcal{C} \otimes \mathcal{D})$

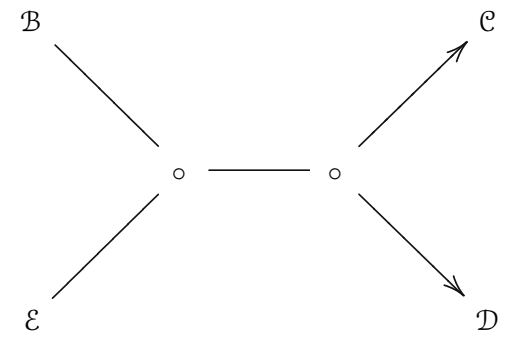

Fig. B.4. Diagram representing an element of $\mathrm{KK}(\mathcal{B} \otimes \mathcal{E}, \mathcal{C} \otimes \mathcal{D})$

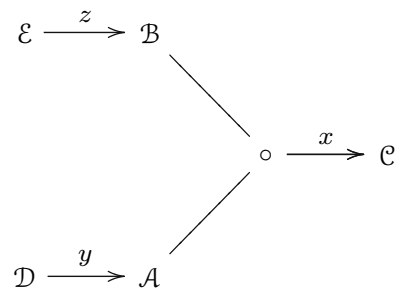

Fig. B.5. Diagram showing that $z \otimes_{\mathcal{B}}\left(y \otimes_{\mathcal{A}} x\right)= \pm y \otimes_{\mathcal{A}}\left(z \otimes_{\mathcal{B}} x\right)$

The associativity of the Kasparov product corresponds to the principle that if one has multiple concatenations to do, the concatenations can be done in any order, except perhaps for keeping track of signs. For example, if

$$
x \in \mathrm{KK}(\mathcal{B} \otimes \mathcal{A}, \mathcal{C}), \quad y \in \mathrm{KK}(\mathcal{D}, \mathcal{A}), \quad \text { and } \quad z \in \mathrm{KK}(\mathcal{E}, \mathcal{B}),
$$

then the associativity of the product gives

$$
z \otimes_{\mathcal{B}}\left(y \otimes_{\mathcal{A}} x\right)= \pm y \otimes_{\mathcal{A}}\left(z \otimes_{\mathcal{B}} x\right),
$$

even though when written this way, it seems to be somewhat counter-intuitive. But one can "prove" this graphically with the picture in Fig. B.5.

Of course, a picture by itself is not a rigorous proof, but it can be made into one as follows. Here $x$ is used to denote the "exterior" Kasparov product, and for simplicity we assume that all elements lie in $\mathrm{KK}_{0}$, so that we don't have to worry about sign changes 
(which all have to do with conventions about orientation of the Bott element). On the one hand, we have

$$
\begin{aligned}
z \otimes_{\mathcal{B}}\left(y \otimes_{\mathcal{A}} x\right): & =\left(z \times 1_{\mathcal{D}}\right) \otimes_{\mathcal{B} \otimes \mathcal{D}}\left(y \otimes_{\mathcal{A}} x\right) \\
& =\left(z \times 1_{\mathcal{D}}\right) \otimes_{\mathcal{B} \otimes \mathcal{D}}\left(\left(1_{\mathcal{B}} \times y\right) \otimes_{\mathcal{B} \otimes \mathcal{A}} x\right) \\
& =\left[\left(z \times 1_{\mathcal{D}}\right) \otimes_{\mathcal{B} \otimes \mathcal{D}}\left(1_{\mathcal{B}} \times y\right)\right] \otimes_{\mathcal{B} \otimes \mathcal{A}} x .
\end{aligned}
$$

But on the other hand we have

$$
\begin{aligned}
y \otimes_{\mathcal{A}}\left(z \otimes_{\mathcal{B}} x\right) & :=\left(1_{\mathcal{E}} \times y\right) \otimes_{\mathcal{E} \otimes \mathcal{A}}\left(z \otimes_{\mathcal{B}} x\right) \\
& =\left(1_{\mathcal{E}} \times y\right) \otimes_{\mathcal{E} \otimes \mathcal{A}}\left(\left(z \times 1_{\mathcal{A}}\right) \otimes_{\mathcal{B} \otimes \mathcal{A}} x\right) \\
& =\left[\left(1_{\mathcal{E}} \times y\right) \otimes_{\mathcal{E} \otimes \mathcal{A}}\left(z \times 1_{\mathcal{A}}\right)\right] \otimes_{\mathcal{B} \otimes \mathcal{A}} x .
\end{aligned}
$$

So to prove the associativity formula, it suffices to observe that

$$
\left(z \times 1_{\mathcal{D}}\right) \otimes_{\mathcal{B} \otimes \mathcal{D}}\left(1_{\mathcal{B}} \times y\right)=z \times y=\left(1_{\mathcal{E}} \times y\right) \otimes_{\mathcal{E} \otimes \mathcal{A}}\left(z \times 1_{\mathcal{A}}\right) .
$$

We should mention incidentally that essentially everything we said about the Kasparov product applies equally well to products in bivariant cyclic homology, whose formal properties are exactly the same.

Acknowledgements We would like to thank P. Bouwknegt, K. Hannabuss, J. Kaminker, R. Plymen, R. Reis and I.M. Singer for helpful discussions. J.B. and R.J.S. were supported in part by the London Mathematical Society. V.M. was supported by the Australian Research Council. J.R. was supported in part by the USA National Science Foundation, grant number DMS-0504212. R.J.S. was supported in part by PPARC Grant PPA/G/S/2002/00478 and by the EU-RTN Network Grant MRTN-CT-2004-005104. J.B., V.M., and J.R. all thank the Erwin Schrödinger International Institute for Mathematical Physics for its hospitality under the auspices of the programme in Gerbes, Groupoids, and Quantum Field Theory, which made part of this collaboration possible.

\section{References}

1. Asakawa, T., Sugimoto, S., Terashima, S.: D-branes, matrix theory and K-homology. JHEP 03, 034 (2002)

2. Atiyah, M.F.: Global theory of elliptic operators. In: Proceedings of the International Symposium on Functional Analysis. Tokyo: University of Tokyo Press, 1969, pp. 21-30

3. Atiyah, M.F., Segal, G.: Twisted $K$-theory. Ukr. Mat. Visn. 1, no. 3, 287-330 (2004); translation in Ukr. Math. Bull. 1, no. 3, 291-334 (2004)

4. Baaj, S., Julg, P.: Théorie bivariante de Kasparov et opérateurs non bornés dans les $C^{*}$-modules hilbertiens. C. R. Acad. Sci. Paris Sér. I Math. 296(21), 875-878 (1983)

5. Blackadar, B.: K-Theory for Operator Algebras, second edition. Math. Sci. Res. Inst. Publ., Vol. 5. Cambridge: Cambridge University Press, 1998

6. Baum, P., Douglas, R.G.: $K$ homology and index theory. In: Kadison, R.V. (ed.), Operator algebras and applications, Part I (Kingston, Ont., 1980), Proc. Sympos. Pure Math., Vol. 38. Providence, R.I.: Amer. Math. Soc., 1982, pp. 117-173

7. Bott, R., Tu, L.W.: Differential forms in algebraic topology. Graduate Texts in Math., Vol. 82. New York: Springer-Verlag, 1982

8. Bouwknegt, P., Carey, A., Mathai, V., Murray, M., Stevenson, D.: Twisted K-Theory and K-Theory of Bundle Gerbes. Commun. Math. Phys. 228(1), 17-49 (2002)

9. Bouwknegt, P., Evslin, J., Mathai, V.: T-duality: Topology change from $H$-flux. Commun. Math. Phys. 249(2), 383-415 (2004)

10. Bouwknegt, P., Evslin, J., Mathai, V.: On the topology and H-flux of T-dual manifolds. Phys. Rev. Lett. 92(18), 181601 (2004)

11. Bouwknegt, P., Hannabuss, K.C., Mathai, V.: T-duality for principal torus bundles. JHEP 03, 018 (2004) 
12. Bouwknegt, P., Hannabuss, K.C., Mathai, V.: Nonassociative tori and applications to T-duality. Commun. Math. Phys. 264(1), 41-69 (2006)

13. Bouwknegt, P., Mathai, V.: D-Branes, B-Fields and Twisted K-Theory. JHEP 03, 007 (2000)

14. Brodzki, J., Plymen, R.: Periodic cyclic homology of certain nuclear algebras. C. R. Acad. Sci. Paris Sér. I Math. 329, 671-676 (1999)

15. Brylinski, J.-L.: Loop Spaces, Characteristic Classes, and Geometric Quantization. Boston: Birkhäuser, 1993

16. Carey, A., Hannabuss, K.C., Mathai, V., McCann, P.: Quantum Hall Effect on the Hyperbolic Plane. Commun. Math. Phys. 190, 629-673 (1998)

17. Choi, M.D., Effros, E.G.: Nuclear $C^{*}$-algebras and the approximation property. Amer. J. Math. 100(1), 61-79 (1978)

18. Cherix, P.-A., Cowling, M., Jolissaint, P., Julg, P., Valette, A.: Groups with the Haagerup property. Gromov's a-T-menability. Progress in Mathematics, Vol. 197. Basel: Birkhäuser Verlag, 2001

19. Connes, A.: An analogue of the Thom isomorphism for crossed products of a $C^{*}$-algebra by an action of $\mathbb{R}$. Adv. in Math. 39(1), 31-55 (1981)

20. Connes, A.: Noncommutative Geometry. New York: Academic Press, 1994

21. Connes, A.: Gravity coupled with matter and the foundation of noncommutative geometry. Commun. Math. Phys. 182, 155-176 (1996)

22. Connes, A., Dubois-Violette, M.: Noncommutative finite-dimensional manifolds. I. Spherical manifolds and related examples. Commun. Math. Phys. 230(3), 539-579 (2002)

23. Connes, A., Landi, G.: Noncommutative manifolds: The instanton algebra and isospectral deformations. Commun. Math. Phys. 221, 141-159 (2001)

24. Connes, A., Moscovici, H.: Cyclic Cohomology, the Novikov Conjecture and Hyperbolic Groups. Topology 29, 345-388 (1990)

25. Connes, A., Moscovici, H.: The Local Index Formula in Noncommutative Geometry. Geom. Funct. Anal. 5, 174-243 (1995)

26. Cuntz, J.: Bivariante $K$-theorie für lokalconvexe Algebren und der bivariante Chern-Connes-Charakter. Doc. Math. 2, 139-182 (1997)

27. Cuntz, J.: Cyclic Theory and the Bivariant Chern character. In: Cyclic Homology in Noncommutative Geometry, Encyclopaedia of Mathematical Sciences, Vol. 121. Berlin: Springer-Verlag, 2004, pp. 1-71

28. Cuntz, J.: Cyclic theory and the bivariant Chern-Connes character. In: Noncommutative Geometry, Lecture Notes in Math., Vol. 1831. Berlin: Springer-Verlag, 2004, pp. 73-135

29. Cuntz, J., Quillen, D.: Cyclic homology and nonsingularity. J. Amer. Math. Soc. 8(2), 373-442 (1995)

30. Cuntz, J., Quillen, D.: Excision in Bivariant Cyclic Cohomology. Invent. Math. 127, 67-98 (1997)

31. Dixmier, J., Douady, A.: Champs continus d'espaces hilbertiens et de $C^{*}$-algèbres. Bull. Soc. Math. France 91, 227-284 (1963)

32. Emerson, H.: Noncommutative Poincaré Duality for boundary actions of hyperbolic groups. J. Reine Angew. Math. 564, 1-33 (2003)

33. Emerson, H.: The Baum-Connes Conjecture, Noncommutative Poincaré Duality and the Boundary of the Free Group. Int. J. Math. Math. Sci. 38, 2425-2445 (2003)

34. Fack, T., Skandalis, G.: Connes' analogue of the Thom isomorphism for the Kasparov groups. Invent. Math. 64(1), 7-14 (1981)

35. Freed, D.: Dirac charge quantization and generalized differential cohomology. In: Surveys in Differential Geometry, VII. Somerville, MA: Int. Press (2000), pp. 129-194; —_: K-Theory in Quantum Field Theory. In: Current Developments in Math. Somerville, MA: Int. Press (2001), pp. 41-87

36. Freed, D., Hopkins, M.J.: On Ramond-Ramond fields and $K$-theory. JHEP 05, 044 (2000)

37. Freed, D., Witten, E.: Anomalies in string theory with D-branes. Asian J. Math. 3(4), 819-851 (1999)

38. Gorokhovsky, A.: Explicit formulae for characteristic classes in noncommutative geometry. Ph.D. thesis, Ohio State University, 1999

39. Gracia-Bondia, J.M., Varilly, J.C., Figueroa, H.: Elements of Noncommutative Geometry, Birkhäuser Advanced Texts. Boston: Birkhäuser, 2001

40. Higson, N., Kasparov, G.: $E$-theory and $K K$-theory for groups which act properly and isometrically on Hilbert space. Invent. Math. 144(1), 23-74 (2001)

41. Higson, N., Roe, J.: Analytic K-homology. Oxford: Oxford University Press, 2000

42. Hilsum, M., Skandalis, G.: Morphismes $K$-orientés d'espaces de feuilles et fonctorialité en théorie de Kasparov (d'après une conjecture d'A. Connes). Ann. Sci. École Norm. Sup. (4) 20(3), 325-390 (1987)

43. Hopkins, M.J., Singer, I.M.: Quadratic functions in geometry, topology, and M-theory. J. Differ. Geom. 70(3), 329-452 (2005)

44. Horava, P.: Type IIA D-Branes, K-Theory, and Matrix Theory. Adv. Theor. Math. Phys. 2, 13731404 (1999)

45. Hori, K.: D-branes, T-duality, and index theory. Adv. Theor. Math. Phys. 3, 281-342 (1999) 
46. Hull, C.: Global Aspects of T-Duality, Gauged Sigma Models and T-Folds. http://arxiv.org/abs/hep-th/ 0604178

47. Jaffe, A., Leśniewski, A., Osterwalder, K.: Quantum K-Theory: The Chern Character. Commun. Math. Phys. 118, 1-14 (1988)

48. Jakob, M.: A Bordism-Type Description of Homology. Manuscripta Math. 96, 67-80 (1998)

49. Jolissaint, P.: Rapidly decreasing functions in reduced $C^{*}$-algebras of groups. Trans. Amer. Math. Soc. 317(1), 167-196 (1990)

50. Kaminker, J., Putnam, I.: K-theoretic duality for shifts of finite type. Commun. Math. Phys. 187, 509-522 (1997)

51. Kasparov, G.G.: The Operator K-Functor and Extensions of $C^{*}$-Algebras. Izv. Akad. Nauk SSSR Ser. Mat. 44(3), 571-636, 719, (1980); Math. USSR Izv. 16, 513-572 (1981)

52. Kasparov, G.G.: $K$-theory, group $C^{*}$-algebras and higher signatures. Conspectus (1980). In: Ferry, S., Ranicki, A., Rosenberg, J. (eds.) Novikov conjectures, index theorems and rigidity, Vol. 1, Lond. Math. Soc. Lecture Note Series 226. Cambridge: Cambridge University Press, 1995, pp. 101-146

53. Kasparov, G.G.: Equivariant $K K$-theory and the Novikov conjecture. Invent. Math. 91, 147-201 (1988)

54. Lance, E.C.: Hilbert $C^{*}$-modules, A toolkit for operator algebraists. Cambridge: Cambridge University Press, 1995

55. Lawson, H.B., Jr., Michelsohn, M.-L.: Spin Geometry. Princeton: Princeton University Press, 1989

56. Li, H.: Flabby Strict Deformation Quantizations and K-Groups. K-Theory 32, 323-329 (2004)

57. Maldacena, J.M., Moore, G.W., Seiberg, N.: D-Brane Instantons and K-Theory Charges. JHEP 11, 062 (2001)

58. Mathai, V.: Heat Kernels and the Range of the Trace on Completions of Twisted Group Algebras. Contemp. Math. 398, 321-346 (2006)

59. Mathai, V., Rosenberg, J.: T-duality for torus bundles with H-fluxes via noncommutative topology. Commun. Math. Phys. 253, 705-721 (2005)

60. Mathai, V., Rosenberg, J.: T-duality for torus bundles with H-fluxes via noncommutative topology, II: the high-dimensional case and the T-duality group. Adv. Theor. Math. Phys. 10(1), 123-158 (2006)

61. Mathai, V., Melrose, R.B., Singer, I.M.: The Index of Projective Families of Elliptic Operators. Geom. Topol. 9, 341-373 (2005)

62. Meyer, R.: Analytic cohomology. Ph.D. Thesis, Univ. of Münster, 1999, available at http://arxiv.org/abs/ math/9906205

63. Minasian, R., Moore, G.W.: K-theory and Ramond-Ramond charge. JHEP 11, 002 (1997)

64. Moore, G.W., Witten, E.: Self-Duality, Ramond-Ramond Fields, and K-Theory. JHEP 05, 032 (2000)

65. Moscovici, H.: Eigenvalue inequalities and Poincaré duality in noncommutative geometry. Commun. Math. Phys. 184(3), 619-628 (1997)

66. Olsen, K., Szabo, R.J.: Constructing D-Branes from $K$-Theory. Adv. Theor. Math. Phys. 3, 889-1025 (1999)

67. Parker, E.M.: Graded continuous trace $C^{*}$-algebras and duality. In: Operator Algebras and Topology (Craiova, 1989), Pitman Res. Notes Math. Ser., Vol. 270, Harlow, UK: Longman Sci. Tech., 1992, pp. 130-145

68. Pimsner, M., Voiculescu, D.: Exact sequences for $K$-groups and Ext-groups of certain cross-product $C^{*}$-algebras. J. Operator Theory 4(1), 93-118 (1980)

69. Plymen, R.J.: Strong Morita Equivalence, Spinors and Symplectic Spinors. J. Operator Theory 16, 305-324 (1986)

70. Ponge, R.: A New Short Proof of the Local Index Formula and Some of its Applications. Commun. Math. Phys. 241, 215-234 (2003)

71. Puschnigg, M.: Explicit product structures in cyclic homology theories. $K$-Theory 15(4), 323-345 (1998)

72. Puschnigg, M.: Excision in cyclic homology theories. Invent. Math. 143(2), 249-323 (2001)

73. Puschnigg, M.: Diffeotopy functors of ind-algebras and local cyclic cohomology. Doc. Math. 8, 143-245 (2003)

74. Quillen, D.G.: Algebra Cochains and Cyclic Cohomology. Publ. Math. IHES 68, 139-174 (1989)

75. Reis, R.M.G., Szabo, R.J.: Geometric K-Homology of Flat D-Branes. Commun. Math. Phys. 266, 71-122 (2006)

76. Rieffel, M.: Induced representations of $C^{*}$-algebras. Adv. Math. 13, 176-257 (1974)

77. Rieffel, M.: $C^{*}$-algebras associated with irrational rotations. Pacific J. Math. 93(2), 415-429 (1981)

78. Rosenberg, J.: Continuous Trace Algebras from the Bundle Theoretic Point of View. J. Austral. Math. Soc. Ser. A 47, 368-381 (1989)

79. Rosenberg, J.: Behavior of $K$-theory under quantization. In: Operator algebras and quantum field theory (Rome, 1996), Cambridge, MA: Internat. Press, 1997, pp. 404-415

80. Rosenberg, J., Schochet, C.: The Künneth theorem and the universal coefficient theorem for Kasparov's generalized $K$-functor. Duke Math. J. 55(2), 431-474 (1987)

81. Shelton, J., Taylor, W., Wecht, B.: Nongeometric Flux Compactifications. JHEP 10, 085 (2005) 
82. Shirbisheh, V.: $K$-theory tools for local and asymptotic cyclic cohomology. Proc. Amer. Math. Soc. 133(4), 1185-1195 (2005)

83. Skandalis, G.: Une notion de nucléarité en $K$-théorie (d'après J. Cuntz). $K$-Theory 1(6), 549-573 (1988)

84. Szabo, R.J.: D-Branes, Tachyons and K-Homology. Mod. Phys. Lett. A17, 2297-2315 (2002)

85. Tu, J-L.: The Baum-Connes conjecture for groupoids. In: $C^{*}$-algebras (Münster, 1999). Berlin: SpringerVerlag, 2000, pp. 227-242

86. Witten, E.: Overview of $K$-theory applied to strings. Int. J. Mod. Phys. A16, 693-706 (2001) D-branes and $K$-theory. JHEP 12, 019 (1998)

Communicated by A. Connes 